\title{
FRONT TRACKING FOR A MODEL OF IMMISCIBLE GAS FLOW WITH LARGE DATA
}

\author{
HELGE HOLDEN, NILS HENRIK RISEBRO, AND HILDE SANDE
}

\begin{abstract}
In this paper we study front tracking for a model of one dimensional, immiscible flow of several isentropic gases, each governed by a gammalaw. The model consists of the $p$-system with variable gamma representing the different gases. The main result is the convergence of a front tracking algorithm to a weak solution, thereby giving existence as well. This convergence holds for general initial data with a total variation satisfying a specific bound. The result is illustrated by numerical examples.
\end{abstract}

\section{INTRODUCTION}

We want to describe the one dimensional, immiscible flow for several isentropic gases. The different gases are initially separated, and the pressure is for all gases given by a $\gamma$-law, that is, $p=\rho^{\gamma}$, where $\rho$ is the density and $\gamma$ is the adiabatic gas constant for each gas. We assume $\gamma(x, t)>1$. In Lagrangian coordinates $\gamma$ only depends on $x$ because the different gases cannot mix. Thus, the flow of these gases is described for $x \in \mathbb{R}$ and $t \in(0, \infty)$ by the system

$$
\begin{aligned}
v_{t}-u_{x} & =0, \\
u_{t}+p(v, \gamma)_{x} & =0, \\
\gamma_{t} & =0,
\end{aligned}
$$

where $v=1 / \rho$ is the specific volume, $u$ is the velocity, and $p(v, \gamma)=v^{-\gamma}$ is the pressure function. This $3 \times 3$ system of hyperbolic conservation laws is strictly hyperbolic for $v<\infty$.

We consider the Cauchy problem for this system, that is, system (1.1) with general initial data

$$
v(x, 0)=v_{0}(x), u(x, 0)=u_{0}(x), \gamma(x, 0)=\gamma_{0}(x), x \in \mathbb{R} .
$$

Glimm 13 proved global existence of a weak solution of the Cauchy problem with initial data of small total variation for strictly hyperbolic systems where each family is either genuinely nonlinear or linearly degenerate, thus including the present system. This solution is found as a limit of the Glimm scheme [13] or of the front tracking method [15, 4. In 16] we extended the existence result to large initial data for 1.1 by using the Glimm scheme. In this paper we prove that a front tracking algorithm converges to a weak solution, thereby giving an alternative existence argument.

System (1.1) is an extension of the $2 \times 2$ system

$$
\begin{aligned}
v_{t}-u_{x} & =0, \\
u_{t}+p(v)_{x} & =0,
\end{aligned}
$$

which describes the flow of one isentropic gas. The parameter $\gamma$ is constant, and the pressure, still given by a $\gamma$-law, is a function of $v$ only. For the $p$-system with $\gamma=1$,

Date: November 5, 2008.

2000 Mathematics Subject Classification. Primary: 35L65, 76N15; Secondary: 35A05.

Key words and phrases. p-system, gamma law, mixture of gases. 
Nishida [20] showed existence of a global weak solution for arbitrary bounded initial data. For $\gamma>1$, Nishida and Smoller 21] proved existence of a weak solution for initial data where $(\gamma-1)$ times the total variation of the initial data is sufficiently small. The case with large initial data for $2 \times 2$ systems is also discussed in [5, 9].

The system (1.1) does not have a coordinate system of Riemann invariants, only a 2-Riemann coordinate. Therefore we do not have the advantage of changing variables to Riemann invariants as for the $p$-system and other $2 \times 2$ systems. Liu 17. proved existence of a solution for the full Euler system with large initial data, another $3 \times 3$ system without a coordinate system of Riemann invariants. Liu's change of variables is inspired by the use of Riemann invariants, but a similar approach does not simplify system (1.1) because $\gamma$ is a function of $x$. The general results by Temple 25] include both the results of 21] and [17. In 25] one considers the flux function as a smooth one-parameter family of functions where one has existence of a solution for initial data in B.V. when $\epsilon=0$. Then the system with $0 \leq \epsilon \leq 1$ has a unique solution if $\epsilon$ times the total variation of the initial data is sufficiently small. Letting $\epsilon=\gamma-1$ for the $p$-system and the Euler equations, one obtains similar results as in 21 and 17. However, this approach cannot be used for system (1.1) since $\gamma$ is one of the variables. Wissman proved in [29] a large data existence theorem for the $3 \times 3$ system of relativistic Euler equations in the ultra-relativistic limit. Applying a change of coordinates the shock waves become translation invariant and a Nishida-type of analysis is used.

For $3 \times 3$ systems with a 2-Riemann coordinate, Temple and Young [26] showed existence of a solution for initial data with arbitrary large total variation, provided that the oscillations are small. This result applies to $(1.1)$ as well, but we want to avoid this restriction on the oscillations. Peng [23, 22] also considered certain $3 \times 3$ systems (Lagrangian gas dynamics for a perfect gas and a model originating in multiphase flow modeling) with large initial data.

All these existence results are proved using the Glimm scheme. Asakura shows the convergence of front tracking for the $p$-system [3] and for the Euler equations [2] with large initial data. The conditions on the initial data are the same as obtained in [21] and [17]. In [7, 8] front tracking is used to study systems of conservation laws whose flux functions depend on a parameter vector, $\mu$, similar to those in 25. An approach for establishing $L^{1}$-estimate pointwise in time between entropy solutions for $\mu \neq 0$ and $\mu=0$ is given. In particular, letting $\mu=\gamma-1$, the $L^{1}$-estimate between entropy solutions in the large for the isentropic Euler equations and the isothermal Euler equations is established in [7] and between entropy solutions in the large for the the Euler equations and the isothermal Euler equations in 8 .

Amadori and Corli [1] extend the $p$-system with an extra equation, $\lambda_{t}=0$, to model multiphase flow, and use front tracking to prove existence of a weak solution for large data. As for system (1.1), the pressure function in [1] is a function of both $v$ and the new variable, $\lambda$, making the two systems similar. However, since the adiabatic gas constant, $\gamma$, is equal to one in [1, vacuum can never occur for their system as it can for system (1.1). Furthermore, the wave curves in 1 are monotone in $\lambda$, resulting in a considerably simpler analysis of the wave interactions compared with the analysis necessary for the model considered here. The system treated in [1] is a simplified version of the model discussed by Fan in [12. Similar models, but with a rather different pressure law, are also considered in 11 and 19 applying completely different methods. A model in the context of the NavierStokes equation with finitely many independent pressure laws has been studied in 6. 
System 1.1 can also be rewritten as a $2 \times 2$ system with discontinuous flux. We get

$$
\begin{aligned}
v_{t}-u_{x} & =0, \\
u_{t}+p(v, \gamma(x))_{x} & =0
\end{aligned}
$$

where the adiabatic gas constant of the different gases is given by the discontinuous function $\gamma(x)$.

This rest of this paper is organized as follows: In Section 2 we discuss the wave curves of the system. The variable $\gamma$ is constant along the rarefaction and shock waves of the first and third family, therefore these curves are similar to the wave curves of the $p$-system. However, these curves are not monotone in $\gamma$, which considerably complicates the interactions of waves with different values of $\gamma$. The second family is linearly degenerate and gives rise to a contact discontinuity along which $p$ and $u$ are constant. Thus, by changing variables to $p, u$ and $\gamma$, the Riemann problem is easy to describe. The invariant region for the Riemann problem includes vacuum. This is a problem since the interaction estimates are not valid when $p$ tends to zero, see 18 .

Section 3 is the main part of this paper where we first present the front-tracking algorithm. The solution of any Riemann problem is made piecewise constant by approximating rarefaction waves as step functions. In addition, a simplified Riemann solver generating non-physical fronts is introduced in order to ensure that the number of fronts remains finite. The simplified solver is only used for interactions where one or more fronts of the same family collide with a contact discontinuity and the sum of the strengths of the incoming fronts times the strength of the contact discontinuity is less than some threshold parameter. This solver generates nonphysical fronts, traveling either to the left or the right, with absolute speed larger than any other front. Moreover, when these non-physical fronts collide with other fronts, they just pass through without changing strength. In Section 3.2 we define a Glimm functional and by considering all possible interactions we prove that it is decreasing under the conditions given in Proposition 3.7. We use this to show that there is a finite number of interactions up to any given time, hence, and thus the front-tracking algorithm is well-defined. Furthermore, we introduce a generation concept in order to bound the total amount of non-physical fronts present at any time. The approximate solution found using front tracking has bounded total variation and is bounded away from vacuum whenever the conditions on the initial data given in Lemma 3.18 and Lemma 3.19 are satisfied. We end Section 3 by proving that the sequence of approximate solutions converges to a weak solution of the system. This proves the main theorem:

Theorem 3.20. Assume that $(\sup (\gamma(\cdot, 0))-1)$ T.V. $(p(\cdot, 0), u(\cdot, 0))$ and T.V. $(\gamma(\cdot, 0))$ are sufficiently small. The the front tracking algorithm is well-defined and gives a sequence which converges to a weak solution of (1.1).

Observe that by reducing the total variation of $\gamma$ and reducing its supremum, one can allow for arbitrary large total variation of $p$ and $u$. Due to Wagner [27, this result translates into existence for the system 3.58 in Eulerian coordinates.

In the last section we study some examples numerically. In the first example we have one gas confined to an interval, surrounded by another gas. The two gases have distinct but constant gammas. The constants that limit the total variation of the initial data are computed, and the initial data are chosen so that they satisfy the conditions in the theorem. The Glimm functional is explicitly computed, and we observe decay in accordance with the theorem. In the second example the initial data are piecewise constant, while $\gamma$ is continuously varying in the third example. 
For these two examples, the total variation of the chosen initial data do not satisfy the theorem, nevertheless we still observe that the Glimm functional is decaying.

\section{THE SYSTEM}

It is well-known that systems of hyperbolic conservation laws such as 11.1 do not in general have smooth solutions, even for smooth initial data. Thus, by a solution of (1.1) with the initial data 1.2 we mean a weak solution in the distributional sense with $(v, u, \gamma) \in L_{\text {loc }}^{1}(\mathbb{R} \times[0, \infty))$ so that

$$
\begin{array}{r}
\iint_{\mathbb{R} \times[0, \infty)}\left(v \phi_{t}-u \phi_{x}\right) \mathrm{d} x \mathrm{~d} t+\int_{\mathbb{R}} v_{0}(x) \phi(x, 0) \mathrm{d} x=0, \\
\iint_{\mathbb{R} \times[0, \infty)}\left(u \phi_{t}+p \phi_{x}\right) \mathrm{d} x \mathrm{~d} t+\int_{\mathbb{R}} u_{0}(x) \phi(x, 0) \mathrm{d} x=0, \\
\iint_{\mathbb{R} \times[0, \infty)} \gamma \phi_{t} \mathrm{~d} x \mathrm{~d} t+\int_{\mathbb{R}} \gamma_{0}(x) \phi(x, 0) \mathrm{d} x=0,
\end{array}
$$

for all test function $\phi \in C_{0}^{\infty}(\mathbb{R} \times[0, \infty))$.

If the specific volume, $v$, becomes infinite, which corresponds to zero density and zero pressure, we have vacuum. At vacuum, the properties of the system change and the methods used here do not apply, therefore we only consider system (1.1) for $v(x, t)<\infty$. Furthermore, we assume $\gamma(x, t)>1$.

We write $U(x, t)=(v(x, t), u(x, t), \gamma(x, t))$. Often we will work with $p$ instead of $v$, and then also write $U(x, t)=(p(x, t), u(x, t), \gamma(x, t))$.

For $v<\infty$, or equivalently, $p>0$, system 1.1 is strictly hyperbolic with eigenvalues

$$
\lambda_{1}=-\lambda, \quad \lambda_{2}=0, \quad \lambda_{3}=\lambda,
$$

where $\lambda:=\sqrt{-p_{v}}=\sqrt{\gamma v^{-\gamma-1}}$, and corresponding eigenvectors

$$
r_{1}=(1, \lambda, 0), \quad r_{2}=\left(-p_{\gamma}, 0, p_{v}\right), \quad r_{3}=(-1, \lambda, 0) .
$$

Note that the eigenvalues and eigenvectors do not depend on $u$. The first and the third family are genuinely nonlinear, while the second family is linearly degenerate. Moreover, the system does not possess a coordinate system of Riemann invariants, but $\gamma$ is a Riemann coordinate for the second family.

Before we turn to solving system (1.1) with general initial data, we need to solve the Riemann problem for (1.1), that is, when the initial data consists of two constant states separated by a jump, cf. (2.21). The solution of the Riemann problem consists of up to three elementary waves, one from each family, and up to two intermediate constant states separating these waves. Thus, we start by looking at the wave curves.

2.1. Wave curves. For the genuinely nonlinear families there are two types of waves; rarefaction waves which are continuous waves of the form $U(x, t)=w(x / t)$ satisfying

$$
\dot{w}(x / t)=r_{j}(w(x / t)), \lambda_{j}(w(x / t))=x / t, \quad j=1,3,
$$

where $\lambda_{j}$ is increasing along the wave, and shock waves which are solutions

$$
U(x, t)= \begin{cases}U_{l}, & \text { if } x<\sigma_{j} t, \\ U_{r}, & \text { if } x>\sigma_{j} t,\end{cases}
$$

satisfying the Rankine-Hugoniot condition

$$
\sigma_{j}\left(U_{r}-U_{l}\right)=f\left(U_{r}\right)-f\left(U_{l}\right), \quad j=1,3,
$$


for a shock velocity $\sigma_{j}$. The admissible shock waves are those satisfying the Lax entropy conditions

$$
\lambda_{j-1}\left(U_{l}\right)<\sigma<\lambda_{j}\left(U_{l}\right), \lambda_{j}\left(U_{r}\right)<\sigma<\lambda_{j+1}\left(U_{r}\right), \quad j=1,3 .
$$

For the linearly degenerate family $j=2$ there is only one type of waves called contact discontinuities. These waves are solutions of the form 2.5 which satisfy the Rankine-Hugoniot condition 2.6) with $\sigma=\lambda_{2}$.

Fix a left state $U_{l}$. For each family the wave curve consists of all states $U$ that can be connected to the given left state by a wave of this family. The rarefaction solution is of the form

$$
U(x, t)= \begin{cases}U_{l}, & \text { if } x<\lambda_{j}\left(U_{l}\right) t \\ w(x / t), & \text { if } \lambda_{j}\left(U_{l}\right) t<x<\lambda_{j}(U) t \\ U, & \text { if } x>\lambda_{j}(U) t\end{cases}
$$

The rarefaction wave curve is the set of all right states $U$ that can be connected to the left state by a rarefaction wave. For system $(1.1)$ these are

$$
\begin{array}{lll}
R_{1}\left(v, U_{l}\right):=\left(v, u_{l}-\frac{2 \sqrt{\gamma_{l}}}{\gamma_{l}-1}\left(v^{\frac{1-\gamma_{l}}{2}}-v_{l}^{\frac{1-\gamma_{l}}{2}}\right), \gamma_{l}\right), & v>v_{l}, \\
R_{3}\left(v, U_{l}\right):=\left(v, u_{l}+\frac{2 \sqrt{\gamma_{l}}}{\gamma_{l}-1}\left(v^{\frac{1-\gamma_{l}}{2}}-v_{l}^{\frac{1-\gamma_{l}}{2}}\right), \gamma_{l}\right), & v<v_{l} .
\end{array}
$$

The shock curves of all right states which can be connected to $U_{l}$ by an admissible shock wave are

$$
\begin{array}{ll}
S_{1}\left(v, U_{l}\right):=\left(v, u_{l}-\left(\left(v_{l}-v\right)\left(v^{-\gamma_{l}}-v_{l}^{-\gamma_{l}}\right)\right)^{1 / 2}, \gamma_{l}\right), & v<v_{l}, \\
S_{3}\left(v, U_{l}\right):=\left(v, u_{l}-\left(\left(v_{l}-v\right)\left(v^{-\gamma_{l}}-v_{l}^{-\gamma_{l}}\right)\right)^{1 / 2}, \gamma_{l},\right), & v>v_{l},
\end{array}
$$

with the shock velocities

$$
\begin{aligned}
& \sigma_{1}\left(U_{l}, U\right)=-\sqrt{\frac{v_{l}^{-\gamma_{l}}-v^{-\gamma_{l}}}{v-v_{l}}}=-\sqrt{\frac{p_{l}-p}{p^{-1 / \gamma_{l}}-p_{l}^{-1 / \gamma_{l}}}}, \\
& \sigma_{3}\left(U_{l}, U\right)=\sqrt{\frac{v^{-\gamma_{l}}-v_{l}^{-\gamma_{l}}}{v_{l}-v}}=\sqrt{\frac{p-p_{l}}{p_{l}^{-1 / \gamma_{l}}-p^{-1 / \gamma_{l}}}},
\end{aligned}
$$

respectively. Note that the shock velocities do not depend on $u$. The curve of all right states that can be connected to $U_{l}$ by a contact discontinuity is

$$
C_{2}\left(\gamma, U_{l}\right):=\left(v_{l}^{\gamma_{l} / \gamma}, u_{l}, \gamma\right), \quad \gamma>1
$$

with the velocity $\sigma_{2}=\lambda_{2}=0$.

Note that $\gamma$ only changes along the contact discontinuities. Furthermore, both $u$ and $p=v^{-\gamma}$ are constant along a contact discontinuity, and we therefore choose to work with $p, u$ and $\gamma$. A shock or a rarefaction curve through $U_{l}$ lies in the plane $\gamma=\gamma_{l}$ and is equal to the corresponding wave curve for the $p$-system (1.3) with $\gamma=\gamma_{l}$. We proceed by defining the wave curves using $p, u$, and $\gamma$, as depicted in Figure 1,

$$
\begin{aligned}
& \Phi_{1}\left(p, U_{l}\right):= \begin{cases}\left(p, u_{l}-r\left(p, p_{l}, \gamma_{l}\right), \gamma_{l}\right), & p<p_{l}, \\
\left(p, u_{l}-s\left(p, p_{l}, \gamma_{l}\right), \gamma_{l}\right), & p>p_{l},\end{cases} \\
& \Phi_{2}\left(\gamma, U_{l}\right):=\left(p_{l}, u_{l}, \gamma\right), \quad \gamma>1 \text {, } \\
& \Phi_{3}\left(p, U_{l}\right):= \begin{cases}\left(p, u_{l}+r\left(p, p_{l}, \gamma_{l}\right), \gamma_{l}\right), & p>p_{l}, \\
\left(p, u_{l}-s\left(p, p_{l}, \gamma_{l}\right), \gamma_{l}\right), & p<p_{l},\end{cases}
\end{aligned}
$$


where

$$
\begin{aligned}
& r\left(p, p_{l}, \gamma_{l}\right):=\frac{2 \sqrt{\gamma_{l}}}{\gamma_{l}-1}\left(p^{\frac{\gamma_{l}-1}{2 \gamma_{l}}}-p_{l}^{\frac{\gamma_{l}-1}{2 \gamma_{l}}}\right), \\
& s\left(p, p_{l}, \gamma_{l}\right):=\left(\left(p_{l}^{-\frac{1}{\gamma_{l}}}-p^{-\frac{1}{\gamma_{l}}}\right)\left(p-p_{l}\right)\right)^{1 / 2} .
\end{aligned}
$$

Recall that if $p=0$, we have vacuum, therefore, the wave curves are only well-

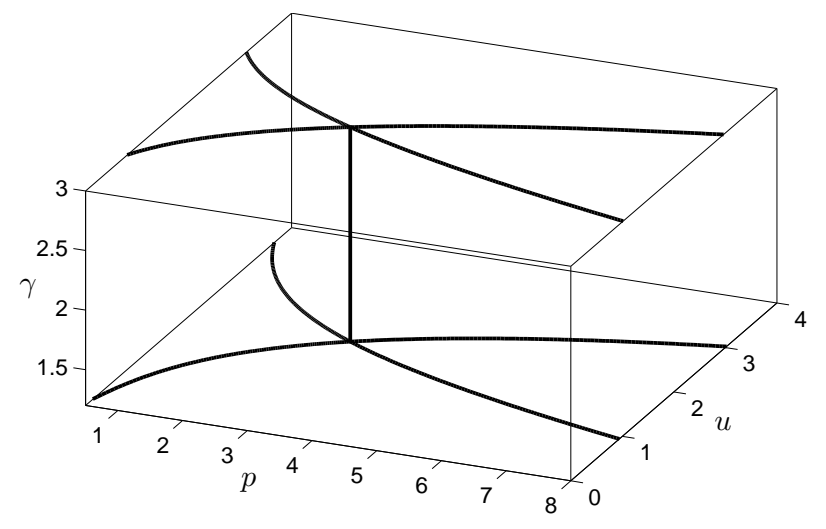

Figure 1. The wave curves through two left states with different $\gamma$.

defined for $p>0$ and $p_{l}>0$. All results are for waves contained in

$$
\mathcal{D}=\left\{(p, u, \gamma)\left|p \in\left[p_{\min }, p_{\max }\right],\right| u \mid<\infty, \gamma \in(1, \bar{\gamma}]\right\}
$$

where $p_{\min }>0, p_{\max }<\infty$ and $\bar{\gamma} \in(1, \infty)$ are constants. For initial data given by 1.2 we will later establish the upper and lower bound on $p$ and show that

$$
\bar{\gamma}:=\sup _{x}\left(\gamma_{0}(x)\right)
$$

for all waves. We moreover have an upper bound on the wave speed for all waves (or fronts) contained in $\mathcal{D}$, and we define

$$
\lambda_{\max }=\max _{U \in \mathcal{D}}\left\{\lambda_{i}, \sigma_{i}\right\}=\max _{U \in \mathcal{D}}\left\{\lambda_{i}\right\}
$$

where the last equality is due to the Lax entropy condition 2.7.

Before we discuss some important properties of the wave curves, we mention the backward wave curves. These are the curves of all left states $U$ that can be connected to a given right state $U_{r}$ by a wave of the given family. We denote these wave curves by $\tilde{\Phi}_{i}$. The backward 3 -wave curve will be used several times and this is given by

$$
\tilde{\Phi}_{3}\left(p, U_{r}\right):= \begin{cases}\left(p, u_{r}-r\left(p_{r}, p, \gamma_{r}\right), \gamma_{r}\right), & p<p_{r} \\ \left(p, u_{r}+s\left(p_{r}, p, \gamma_{r}\right), \gamma_{r}\right), & p>p_{r}\end{cases}
$$

where $r$ and $s$ are given by 2.14 and 2.15. We now turn to the properties of the wave curves.

Lemma 2.1. The wave curves in $\mathcal{D}$ have the following properties:

(i) The function $\Phi_{1}$ is strictly decreasing and the function $\Phi_{3}$ is strictly increasing when considered as functions of $p$.

(ii) Given two wave curves, $\Phi_{j}\left(p, U_{1}\right)$ and $\Phi_{j}\left(p, U_{2}\right)$ where $j \in\{1,3\}$, so that $U_{1}$ is not on $\Phi_{j}\left(p, U_{2}\right)$ and $U_{2}$ is not on $\Phi_{j}\left(p, U_{1}\right)$. Then the two wave curves never intersect. 
(iii) Consider the projections onto the $(p, u)$-plane of the wave curves through $U_{1}=$ $\left(p_{l}, u_{l}, \gamma_{1}\right)$ and $U_{1}=\left(p_{l}, u_{l}, \gamma_{2}\right)$ where $\gamma_{1} \leq \gamma_{2}$. If

$$
\frac{\partial}{\partial p} r\left(p_{l}, p_{l}, \gamma_{1}\right)<\frac{\partial}{\partial p} r\left(p_{l}, p_{l}, \gamma_{2}\right)
$$

then the projected wave curves going to the right (with respect to $p$ ) will never intersect, while the projected wave curves going to the left will intersect as $p$ decreases. If

$$
\frac{\partial}{\partial p} r\left(p_{l}, p_{l}, \gamma_{1}\right)>\frac{\partial}{\partial p} r\left(p_{l}, p_{l}, \gamma_{2}\right)
$$

then the projected wave curves going to the right will intersect, while the projected wave curves going to the left will not. If

$$
\frac{\partial}{\partial p} r\left(p_{l}, p_{l}, \gamma_{1}\right)=\frac{\partial}{\partial p} r\left(p_{l}, p_{l}, \gamma_{2}\right)
$$

then none of the projected wave curves will intersect.

(iv) The slope of a rarefaction wave in the plane $\gamma=\gamma_{l}$, $\partial r / \partial p$, only depends on $p$ and $\gamma_{l}$, not on $p_{l}$. Furthermore, there exist two constants $r_{\min }^{\prime}$ and $r_{\max }^{\prime}$ only depending on $p_{\min }, p_{\max }$ and $\bar{\gamma}$ so that

$$
r_{\min }^{\prime} \leq \frac{\partial}{\partial p} r\left(p, p_{l}, \gamma_{l}\right) \leq r_{\max }^{\prime} .
$$

(v) The slope of a shock wave in the plane $\gamma=\gamma_{l}, \partial s / \partial p$, depends on $p, \gamma_{l}$ and $p_{l}$. Furthermore, there exist two constants $s_{\min }^{\prime}$ and $s_{\max }^{\prime}$ only depending on $p_{\min }, p_{\max }$ and $\bar{\gamma}$ so that

$$
s_{\min }^{\prime} \leq \frac{\partial}{\partial p} s\left(p, p_{l}, \gamma_{l}\right) \leq s_{\max }^{\prime} .
$$

(vi) The wave curves have a continuous derivative at $U_{l}$,

$$
\lim _{p \rightarrow p_{l}} \frac{\partial}{\partial p} s\left(p, p_{l}, \gamma_{l}\right)=\frac{\partial}{\partial p} r\left(p_{l}, p_{l}, \gamma_{l}\right) .
$$

Furthermore,

$$
\frac{\partial}{\partial p} s\left(p, p_{l}, \gamma_{l}\right) \geq \frac{\partial}{\partial p} r\left(p, p_{l}, \gamma_{l}\right)
$$

for all $p_{l}$. Hence, a shock wave is always steeper than a rarefaction wave at a given $p \neq p_{l}$ provided both waves lie in the plane $\gamma=\gamma_{l}$.

(vii) Rarefaction waves are additive; if a rarefaction wave connects $U_{1}$ to $U_{2}$ and another rarefaction wave of the same family connects $U_{2}$ to $U_{3}$, then the rarefaction wave connecting $U_{1}$ to $U_{3}$ equals the concatenation of the other two rarefaction waves.

(viii) Given two 1-shock waves starting at $\left(p_{1}, u, \gamma\right)$ and $\left(p_{2}, u, \gamma\right)$, respectively, and assume $p_{1}<p_{2}$. Then the shock wave starting at $p_{1}$ is steeper than the shock wave starting at $p_{2}$ at any given point $p$, that is,

$$
\frac{\partial}{\partial p} s\left(p, p_{2}, \gamma\right)<\frac{\partial}{\partial p} s\left(p, p_{1}, \gamma\right)
$$

for all $p \geq p_{2}>p_{1}$.

(ix) Given two 3-shock waves starting at $\left(p_{1}, u, \gamma\right)$ and $\left(p_{2}, u, \gamma\right)$, respectively, and assume $p_{1}<p_{2}$. Then the shock wave starting at $p_{2}$ is steeper than the shock wave starting at $p_{1}$ at any given point $p$, that is,

$$
\frac{\partial}{\partial p} s\left(p, p_{1}, \gamma\right)<\frac{\partial}{\partial p} s\left(p, p_{2}, \gamma\right)
$$

for all $p \leq p_{1}<p_{2}$. 
Proof. All the properties follows from differentiating the wave curves.

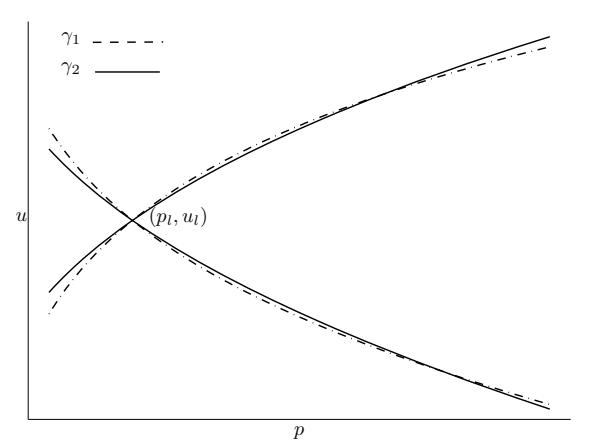

(a) Because $\frac{\partial}{\partial p} r\left(p_{l}, p_{l}, \gamma_{l}\right)>\frac{\partial}{\partial p} r\left(p_{l}, p_{l}, \gamma_{2}\right)$, the projected wave curves going to the right intersect.

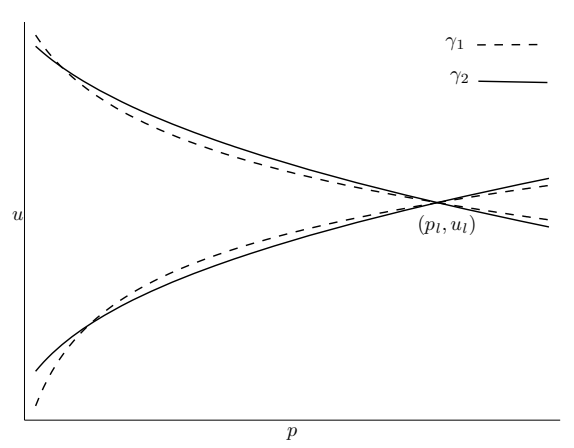

(b) Because $\frac{\partial}{\partial p} r\left(p_{l}, p_{l}, \gamma_{l}\right)<\frac{\partial}{\partial p} r\left(p_{l}, p_{l}, \gamma_{2}\right)$, the projected wave curves going to the left intersect.

Figure 2. The wave curves through $U_{1}=\left(p_{l}, u_{l}, \gamma_{1}\right)$ (dotted line) and $U_{2}=\left(p_{l}, u_{l}, \gamma_{2}\right)$, where $\gamma_{1}<\gamma_{2}$, projected onto the $(p, u)$ plane.

The projection onto the $(p, u)$-plane of two wave curves with different $\gamma$ 's are shown in Figure 2. Note that the projected wave curves intersect, cf. property (iii), because the slopes of the projected wave curves depend on $\gamma$. The next lemma gives an estimate on how different two waves with different $\gamma$ 's are.

Lemma 2.2. Let $\epsilon_{1}$ and $\epsilon_{2}$ be 1-waves of the same type such that $\epsilon_{1}$ connects $\left(p_{0}, u_{0}, \gamma_{1}\right)$ to $\left(p, u_{1}, \gamma_{1}\right)$ and $\epsilon_{2}$ connects $\left(p_{0}, u_{0}, \gamma_{2}\right)$ to $\left(p, u_{2}, \gamma_{2}\right)$, or let $\eta_{1}$ and $\eta_{2}$ be 3-waves of the same type such that $\eta_{1}$ connects $\left(p, u_{1}, \gamma_{1}\right)$ to $\left(p_{0}, u_{0}, \gamma_{1}\right)$ and $\eta_{2}$ connects $\left(p, u_{2}, \gamma_{2}\right)$ to $\left(p_{0}, u_{0}, \gamma_{2}\right)$. Assume that all waves are contained in $\mathcal{D}$ and furthermore that $u_{1}<u_{2}$. Then

$$
u_{2}-u_{1} \leq c_{2}\left|p-p_{0}\right|\left|\gamma_{2}-\gamma_{1}\right|
$$

where $c_{2}$ only depends on $p_{\min }, p_{\max }$ and $\bar{\gamma}$.

Note that for 1-waves we compare two waves where the projected waves start at the same point in the $(p, u)$-plane, while we for 3 -waves compare two waves where the projected waves end at the same point. The proof of this lemma is given in [16] and is based on the techniques used in 28 .

2.2. The Riemann Problem. We have the following fundamental definition.

Definition 2.3. The Riemann problem for (1.1) is the Cauchy problem with initial data

$$
U(x, 0)= \begin{cases}U_{l}, & \text { if } x<0, \\ U_{r}, & \text { if } x>0,\end{cases}
$$

where $U=(v, u, \gamma)$ and $U_{l}, U_{r} \in \mathbb{R}$ are constants.

Lemma 2.4. The Riemann problem for 1.1) where $U_{l}$ and $U_{r}$ are contained in $\mathcal{D}$, cf. 2.16, has a unique solution without vacuum if

$$
u_{r}-u_{l}<r\left(p_{r}, 0, \gamma_{r}\right)-r\left(0, p_{l}, \gamma_{l}\right) .
$$


Proof. Note that if $\gamma_{l}=\gamma_{r}$, then the Riemann problem for 1.1 reduces to the Riemann problem for the $p$-system 1.3 . The solution of this problem is described in detail in [24, Ch. 17, $\S \mathrm{A}]$, and it is unique if [2.22 is satisfied with $\gamma_{l}=\gamma_{r}$.

A 2-wave takes us from one plane, $\gamma=\gamma_{1}$, to another plane, $\gamma=\gamma_{2}$, while $p$ and $u$ remain constant. Therefore, the Riemann problem has a unique solution if the projections onto the $(p, u)$-plane of the 1-wave curve, $\Phi_{1}\left(p, U_{l}\right)$, and the backward 3-wave curve, $\tilde{\Phi}_{3}\left(p, U_{r}\right)$, have a unique intersection point. From property (i) of Lemma 2.1 we have that the projection of $\Phi_{1}$ is strictly decreasing in $p$ and it follows that the projection of $\tilde{\Phi}_{3}$ is strictly increasing in $p$. Hence, the projected curves intersect at most once. The only case where the two curves do not intersect is if the projection of the backward 3-rarefaction wave from $U_{r}$ always lies above the projection of the 1-rarefaction wave from $U_{l}$. Thus, if

$$
u_{r}-r\left(p_{r}, 0, \gamma_{r}\right)<u_{l}-r\left(0, p_{l}, \gamma_{l}\right)
$$

then the projections of $\tilde{\Phi}_{3}\left(p, U_{r}\right)$ and $\Phi_{1}\left(p, U_{l}\right)$ onto the $(p, u)$-plane have a unique intersection point, and the Riemann problem has a unique solution.

The solution of the Riemann problem $\left(U_{l}, U_{r}\right)$ is constructed as follows: Let $(\tilde{p}, \tilde{u})$ be the unique intersection between the projections of $\Phi_{1}\left(p, U_{l}\right)$ and $\tilde{\Phi}_{3}\left(p, U_{r}\right)$ onto the $(p, u)$-plane. We connect $U_{l}=\left(p_{l}, u_{l}, \gamma_{l}\right)$ to $\tilde{U}_{1}=\left(\tilde{p}, \tilde{u}, \gamma_{l}\right)$ by a 1-curve, then we go from $\tilde{U}_{1}$ to $\tilde{U}_{2}=\left(\tilde{p}, \tilde{u}, \gamma_{r}\right)$ along a contact discontinuity, and finally connect $\tilde{U}_{2}$ to $U_{r}=\left(p_{r}, u_{r}, \gamma_{r}\right)$ by a 3 -wave.

2.3. Invariant region and vacuum. A region $\Omega$ is invariant for the Riemann problem if for any Riemann problem with initial data in $\Omega$, its solution is also in $\Omega$. For the $p$-system we know from [14, Ex. 3.5] that the convex region in the $(v, u)$-plane between the integral curves of the eigenvectors is invariant. This region bounds $v$ from below, but not from above, thus vacuum is included in the invariant region. In the $(p, u)$-plane this corresponds to the region bounded by $p=0$ and the two integral curves. Since $\gamma$ cannot take any other values than those of the initial data, we find the invariant region for the $p$-system for each $\gamma$ and take the union of these. This gives us an invariant region for 1.1. Moreover, this gives us the upper bound on $p, p_{\max }$, which we need, but $p$ is still not bounded away from vacuum.

\section{The Cauchy problem}

We now turn to the Cauchy problem and use front tracking to obtain a sequence of approximate solutions. The goal of this section is to show that a subsequence converges to a weak solution of 1.1 . In order to do this, we find a suitable Glimm functional and show that it decreases in time. This requires detailed analysis of all possible interactions and most of this section is devoted to this. First of all we need some notation. We let

$$
\begin{array}{lll}
\epsilon \text { define a 1-wave, } & \alpha \text { a 1-shock wave, } & \mu \text { a } 1 \text {-rarefaction wave, } \\
\eta \text { a } 3 \text {-wave, } & \beta \text { a 3-shock wave, } & \nu \text { a } 3 \text {-rarefaction wave, } \\
\zeta \text { a } 2 \text {-wave } & \theta \text { a } 1 \text { - or 3-wave, } &
\end{array}
$$

Furthermore, we define the strength of a 1-wave or a 3-wave as the jump in $p$ across the wave and the strength of a 2-wave as the jump in $\gamma$ across the wave. The strength of a wave or a front is denoted by $|\theta|$. We are now ready to discuss front tracking and to define fronts. Note that we will use the above notation for fronts as well as waves. In addition, we will define non-physical fronts which will be denoted by $\theta^{\text {np }}$ and the strength of a non-physical front will be defined as its jump in $u$. 
3.1. Front tracking. The first step of front tracking is to approximate the initial data 1.2 by a piecewise constant function $U_{0}^{\delta_{\text {init }}}$ so that

$$
\lim _{\delta_{\text {init }} \rightarrow 0}\left\|U_{0}^{\delta_{\text {init }}}-U_{0}\right\|_{L^{1}}=0,
$$

where $U_{0}=\left(p_{0}, u_{0}, \gamma_{0}\right)$ and $\delta_{\text {init }}$ is the distance between the discontinuities. Furthermore, the approximation has to satisfy 2.22 at every discontinuity so that all initial Riemann problems have a unique solution. Thus, no vacuum forms at $t=0+$.

We then solve the Riemann problem defined by the discontinuities in $U_{0}^{\delta_{\text {init }}}$. All solutions of Riemann problems in front tracking have to be piecewise constant. Since shock waves and contact discontinuities are already piecewise constants, we use an approximate Riemann solver where the continuous rarefaction waves are approximated. We replace the rarefaction wave from the left state, $U_{l}$, to the right state, $U_{r}$, by a step function. Let $k:=\left\lceil\left|p_{r}-p_{l}\right| / \delta\right\rceil$. Then we divide the rarefaction wave into $k$ jumps, each with strength $\hat{\delta}=|\theta| / k \leq \delta$. The discontinuities move with the speed of their left state. Note that the jumps in the approximated rarefaction wave do not satisfy the Rankine-Hugoniot condition. It is obvious that this approximate solution of the Riemann problem converges to the exact solution a.e. when $\delta$ tends to zero.

Solving all Riemann problems present initially by the approximate solver, generates an approximate solution of the Cauchy problem for small $t>0$. The solution is piecewise constant and a front is one discontinuity in the solution. Hence, a shock wave or a contact discontinuity is one front, while an approximated rarefaction wave consists of $k$ fronts where each front has strength less than or equal to $\delta$. Note that the two parameters $\delta_{\text {init }}$ and $\delta$ are chosen so that $\delta_{\text {init }}=\mathcal{O}(\delta)$. We denote the approximate solution $U^{\delta}$.

We track all fronts in $U^{\delta}$ until two or more fronts interact, that is, collide at a collision point $(x, \tau)$. The colliding fronts are called incoming fronts. Then we solve the Riemann problem defined by the states immediately to the left and right of the incoming fronts, and the fronts in this approximate solution are called outgoing fronts and are usually identifiable by a prime. We keep tracking all fronts and solving Riemann problems each time fronts collide.

In order to ensure that front tracking is well-defined for all times, we follow the approach of Bressan [4] and introduce non-physical fronts. Thus, an interaction is either solved by the standard approximate solver as described above, or by a simplified Riemann solver. Let $\rho>0$ be a fixed threshold parameter. Interactions of the form $\zeta+\sum_{i} \epsilon_{i}$, or the symmetric form $\sum_{i} \eta_{i}+\zeta$, are solved using the simplified Riemann solver if

$$
|\zeta| \sum_{i}\left|\epsilon_{i}\right| \leq \rho, \text { or }|\zeta| \sum_{i}\left|\eta_{i}\right| \leq \rho
$$

respectively, otherwise the approximate Riemann solver is used. All other interactions are always solved using the approximate solver. The simplified Riemann solver introduces non-physical fronts which we denote $\theta^{\text {np }}$. By construction, both $p$ and $\gamma$ are constant across a non-physical front and its strength equals the jump in $u$. In order to preserve the symmetry property of system (1.1), we introduce non-physical fronts traveling both to the left and to the right. In either case they travel with the absolute speed $\lambda_{\mathrm{np}}>\lambda_{\max }$, hence the name. Note that the Rankine-Hugoniot condition (2.6) is not satisfied for a non-physical front.

Let us first detail the solution of the interaction between one front and a contact discontinuity using the simplified solver. The solution consists of two physical fronts and a non-physical front:

$$
\zeta+\epsilon \rightarrow \epsilon^{\prime}+\zeta+\theta^{\text {np }}
$$


The outgoing front $\epsilon^{\prime}$ has the same strength and type as $\epsilon$, and connects $U_{l}=$ $\left(p_{l}, u_{l}, \gamma_{l}\right)$ to $\tilde{U}_{1}=\left(p_{r}, \tilde{u}, \gamma_{l}\right)$, as depicted in Figure $3(\mathrm{a})$ The contact discontinuity is, as always, unchanged, connecting $\tilde{U}_{1}$ to $\tilde{U}_{2}=\left(p_{r}, \tilde{u}, \gamma_{r}\right)$. The non-physical front then connects $\tilde{U}_{2}$ to $U_{r}=\left(p_{r}, u_{r}, \gamma_{r}\right)$. Moreover, the non-physical front has positive speed traveling to the right. For the symmetric case,

$$
\eta+\zeta \rightarrow \theta^{\mathrm{np}}+\zeta+\eta^{\prime}
$$

the non-physical front has negative speed.

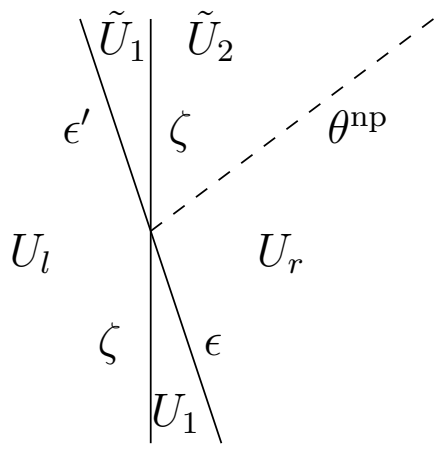

(a) Simplified solver for $\zeta+\epsilon$.

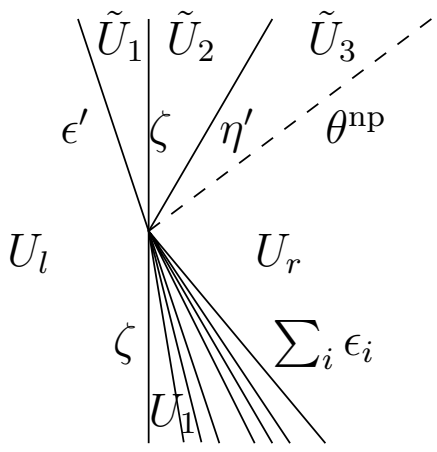

(b) Simplified solver for $\zeta+\sum_{i} \epsilon_{i}$.

FiguRE 3. The simplified Riemann solver with non-physical fronts (dashed lines).

The solution we get using the simplified solver when two or more fronts of the same family interact with a contact discontinuity, consists of one physical front of each family, in addition to a non-physical front;

$$
\zeta+\sum_{i} \epsilon_{i} \rightarrow \epsilon^{\prime}+\zeta+\eta^{\prime}+\theta^{\mathrm{np}}
$$

see Figure 3(b) In order to determine the outgoing fronts, we introduce two auxiliary fronts, $\bar{\epsilon}$ and $\bar{\eta}$. These fronts are the solution of the Riemann problem $\left(U_{1}, U_{r}\right)$, thus, $\bar{\epsilon}$ connects $U_{1}$ to the intermediate state $\bar{U}=\left(\bar{p}, \bar{u}, \gamma_{r}\right)$, and $\bar{\eta}$ connects $\bar{U}$ to $U_{r}$. Let $\epsilon^{\prime}$ be the front that has the same strength and type as $\bar{\epsilon}$, but with $\gamma=\gamma_{l}$, that is, connecting $U_{l}$ to $\tilde{U}_{1}=\left(\bar{p}, \tilde{u}, \gamma_{l}\right)$. The contact discontinuity is unchanged, connecting $\tilde{U}_{1}$ to $\tilde{U}_{2}=\left(\bar{p}, \tilde{u}, \gamma_{r}\right)$. Let $\eta^{\prime}$ be $\bar{\eta}$ shifted in the $u$-direction so that $\eta^{\prime}$ connects $\tilde{U}_{2}$ to $\tilde{U}_{3}=\left(p_{r}, u_{r}+\tilde{u}-\bar{u}, \gamma_{r}\right)$. Finally, the non-physical front connects $\tilde{U}_{3}$ to $U_{r}$. The non-physical front has positive speed and changes only the value of $u$, as it is supposed to. This construction of the solution using the simplified Riemann solver is inspired by the formal tool of splitting an interaction into steps that we will introduce in the next section. More details on the process of finding the outgoing fronts using the simplified solver are included in the proof of Lemma 3.11 where we obtain estimates for these interactions. Note that $\epsilon^{\prime}+\zeta+\eta^{\prime}$ is the solution of the Riemann problem $\left(U_{l}, \tilde{U}_{3}\right)$, thus, the Rankine-Hugoniot condition 2.6 is satisfied for any shock or contact discontinuity. However, it is not satisfied for the nonphysical front or any approximated rarefaction wave. We resolve the symmetric interaction in a similar manner, and get a non-physical front with negative speed;

$$
\sum_{i} \eta_{i}+\zeta \rightarrow \theta^{\mathrm{np}}+\epsilon^{\prime}+\zeta+\eta^{\prime}
$$




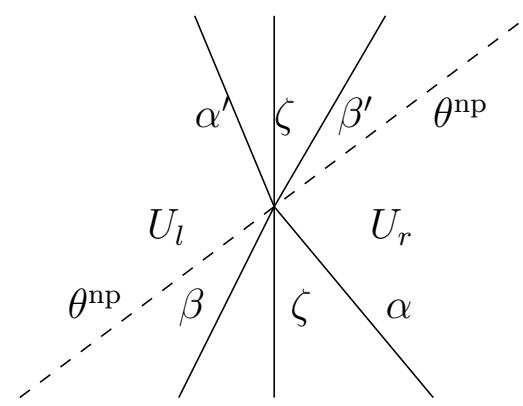

(a) in the $(x, t)$-plane.

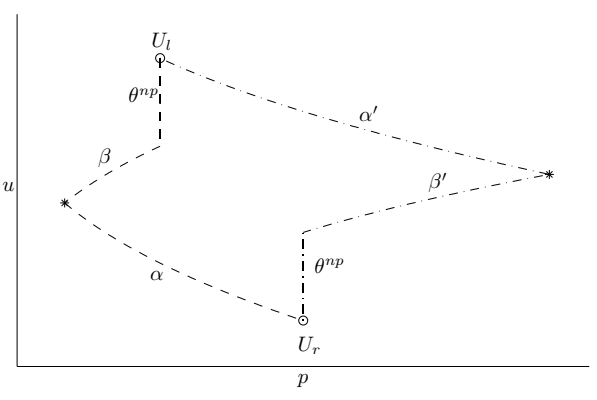

(b) Projected onto the $(p, u)$-plane.

Figure 4. The interaction $\theta^{\mathrm{np}}+\beta+\zeta+\alpha \rightarrow \alpha^{\prime}+\zeta+\beta^{\prime}+\theta^{\mathrm{np}}$.

Whenever we have an interaction with an incoming non-physical front, as in Figure 4, we first let the non-physical front pass through with its strength unchanged. Then we solve the remaining interaction, which is slightly shifted along the $u$-direction, using the approximate or simplified solver according to condition (3.1). Note that all wave curves are invariant in the $(p, u)$ plane under a translation in $u$.

Before we turn to the discussion of all possible interactions, we look at the error introduced using the simplified solver instead of the approximate solver. The lemma is given for the interactions involving 1-fronts, but we have the same results for the symmetric interactions involving 3 -fronts.

Lemma 3.1. Consider the interaction $\zeta+\sum_{i}^{n} \epsilon_{i}$ for $n \geq 1$. Let

$$
\zeta+\sum_{i}^{n} \epsilon_{i} \rightarrow \hat{\epsilon}+\zeta+\hat{\eta}
$$

be the solution, with intermediate states $\hat{U}_{i}, i=1,2$, obtained using the approximate solver, and let

$$
\zeta+\sum_{i}^{n} \epsilon_{i} \rightarrow \begin{cases}\epsilon^{\prime}+\zeta+\theta^{n p}, & \text { if } n=1, \\ \epsilon^{\prime}+\zeta+\eta^{\prime}+\theta^{n p}, & \text { if } n>1,\end{cases}
$$

be the solution obtained using the simplified solver, with intermediate states $\tilde{U}_{i}$, $i=1,2$ and $i=1,2,3$, respectively. Then,

$$
\begin{aligned}
\left|\sigma_{\hat{\alpha}}-\sigma_{\alpha^{\prime}}\right| & =\mathcal{O}(1)\left|\theta^{n p}\right|, \text { if } \epsilon^{\prime}=\alpha^{\prime}, \\
\left|\lambda_{\hat{\mu}}-\lambda_{\mu^{\prime}}\right| & =0, \text { if } \epsilon^{\prime}=\mu^{\prime},
\end{aligned}
$$

and, if $\hat{\eta}$ is of the same type as $\eta^{\prime}$ for $n>1$,

$$
\begin{aligned}
\left|\sigma_{\hat{\beta}}-\sigma_{\beta^{\prime}}\right| & =\mathcal{O}(1)\left|\theta^{n p}\right|, \text { if } \eta^{\prime}=\beta^{\prime}, \\
\left|\lambda_{\hat{\nu}}-\lambda_{\nu^{\prime}}\right| & =\mathcal{O}(1)\left|\theta^{n p}\right|, \text { if } \eta^{\prime}=\nu^{\prime} .
\end{aligned}
$$

Moreover, $\left|\hat{U}_{i}-\tilde{U}_{i}\right|=\mathcal{O}(1)\left|\theta^{n p}\right|, i=1,2$, and $\left|U_{r}-\tilde{U}_{j}\right|=\mathcal{O}(1)\left|\theta^{n p}\right|$ where $j=2$ if $n=1$ and $j=3$ if $n>1$.

Proof. First note that $p$ and $u$ are equal for $\tilde{U}_{1}$ and $\tilde{U}_{2}$, and for $\hat{U}_{1}$ and $\hat{U}_{2}$, and we therefore omit the indices. Figure 5 shows the solutions of $\zeta+\epsilon$ for both solvers, and Figure 6 shows the solutions and the auxiliary fronts for an interaction of the type $\zeta+\sum_{i} \epsilon_{i}$. The rarefaction fronts $\mu^{\prime}$ and $\hat{\mu}$ have the same left state, and they therefore have the same speed. Likewise, the left state is the same for $\alpha^{\prime}$ 


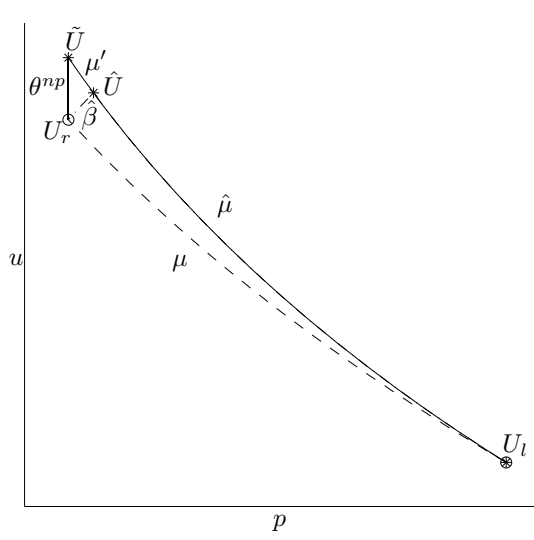

(a) $\zeta+\mu$.

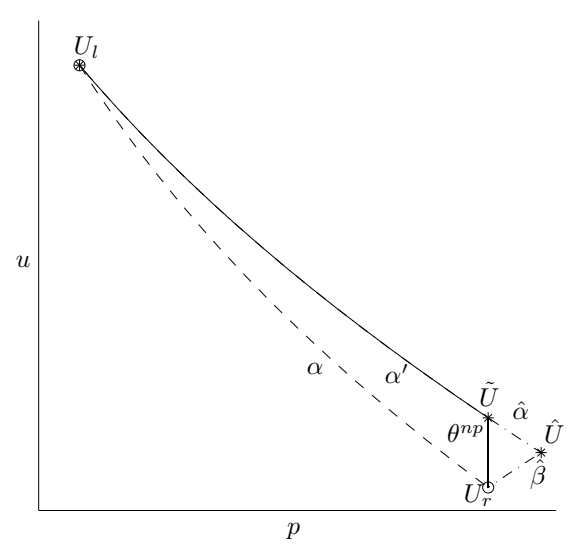

(b) $\zeta+\alpha$.

FiguRE 5. The interaction (dashed lines) solved by the approximate solver (dash-dotted lines) and by the simplified solver (solid lines).

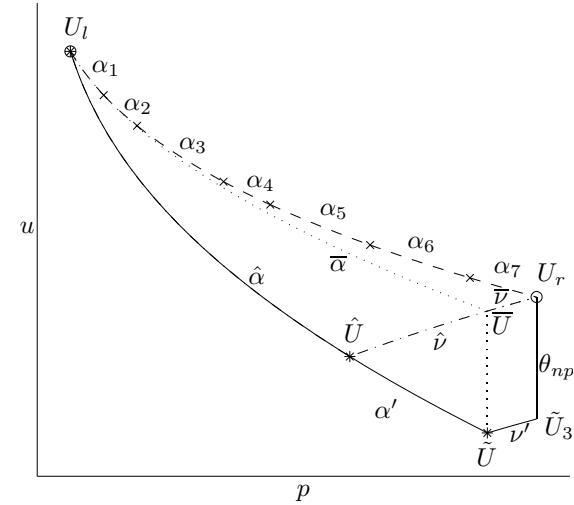

(a) When $\eta^{\prime}$ and $\hat{\eta}$ are of the same type

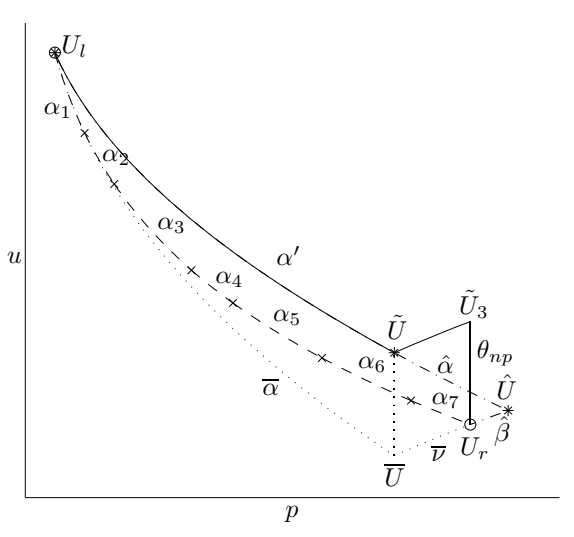

(b) When $\eta^{\prime}$ and $\hat{\eta}$ are not of the same type.

FiguRE 6. The interaction $\zeta+\sum_{i=1}^{7} \alpha_{i}$ (dashed lines), with the auxiliary curves (dotted lines), solved by the approximate solver (dash-dotted lines) and by the simplified solver (solid lines).

and $\hat{\alpha}$. However, the speed of a shock-front depends on the value of $p$ at the right state as well, where $p=\tilde{p}$ for $\alpha^{\prime}$ and $p=\hat{p}$ for $\hat{\alpha}$. Since this difference in $p$ is less than a constant times the jump in $u$ across the non-physical front, that is, $|\tilde{p}-\hat{p}|=\mathcal{O}(1)\left|\theta^{\text {np }}\right|$, we get

$$
\left|\sigma_{\hat{\alpha}}-\sigma_{\alpha^{\prime}}\right|=\left|\sigma_{1}\left(p_{l}, \hat{p}\right)-\sigma_{1}\left(p_{l}, \tilde{p}\right)\right| \leq\left|\sigma_{1}^{\prime}\left(p_{l}, p^{*}\right)\right||\tilde{p}-\hat{p}|=\mathcal{O}(1)\left|\theta^{\mathrm{np}}\right|,
$$

where $\sigma_{1}^{\prime}$ is the derivative with respect to the second argument and $\hat{p} \leq p^{*} \leq \tilde{p}$.

If $n>1$ and $\eta^{\prime}$ is of the same type as $\hat{\eta}$, as for the interaction depicted in Figure 6(a), then $p=p_{r}$ at the right state for both fronts. However, at the left state we have $p=\tilde{p}$ for $\eta^{\prime}$ and $p=\hat{p}$ for $\hat{\eta}$. This is the same difference in $p$ as 
above, thus,

$$
\begin{aligned}
\left|\sigma_{\hat{\beta}}-\sigma_{\beta^{\prime}}\right| & =\left|\sigma_{3}\left(\hat{p}, p_{r}\right)-\sigma_{3}\left(\tilde{p}, p_{r}\right)\right| \leq\left|\sigma_{3}^{\prime}\left(p^{*}, p_{r}\right)\right||\tilde{p}-\hat{p}|=\mathcal{O}(1)\left|\theta^{\mathrm{np}}\right|, \\
\left|\lambda_{\hat{\nu}}-\lambda_{\nu^{\prime}}\right| & =|\lambda(\hat{p})-\lambda(\tilde{p})| \leq\left|\lambda_{3}^{\prime}\left(p^{*}\right)\right||\tilde{p}-\hat{p}|=\mathcal{O}(1)\left|\theta^{\mathrm{np}}\right|,
\end{aligned}
$$

where $\sigma_{3}^{\prime}$ is the derivative with respect to the first argument, $\lambda_{3}^{\prime}$ the derivative with respect to $p$, and $\hat{p} \leq p^{*} \leq \tilde{p}$.

Moreover, $\gamma$ is equal for the two solutions and $|\tilde{u}-\hat{u}| \leq\left|\theta^{\text {np }}\right|$, thus, $\left|\hat{U}_{i}-\tilde{U}_{i}\right|=$ $\mathcal{O}(1)\left|\theta^{\text {np }}\right|, i=1,2$. Finally, let $j=2$ for $n=1$ and $j=3$ for $n>1$. Then, $\tilde{p}_{j}=p_{r}$ and $\left|\tilde{u}_{j}-u_{r}\right|=\left|\theta^{\mathrm{np}}\right|$, hence, $\left|U_{r}-\tilde{U}_{j}\right|=\mathcal{O}(1)\left|\theta^{\mathrm{np}}\right|$.

In front tracking an interaction is a collision of arbitrarily many fronts at one point in space-time. However, in order to collide at the same point, their speeds must decrease from left to right. This observation has the immediate consequence.

Lemma 3.2. All interactions between physical fronts in front tracking for system (1.1) is of the general form

$$
\sum_{i=1}^{m} \eta_{i}+\zeta+\sum_{j=1}^{n} \epsilon_{j},
$$

where $\eta_{i}$ is a 3-front, $\zeta$ is a contact discontinuity, $\epsilon_{j}$ is a 1-front, and two adjacent fronts cannot both be rarefaction-fronts. All interactions with incoming non-physical fronts are of the same general form with a non-physical front as the leftmost and/or the rightmost incoming front. Furthermore, all wave families do not need to be present in an interaction.

This is a major difference between front tracking and the Glimm scheme where at most four waves can interact. Furthermore, only the case with two interacting fronts or waves is the same in front tracking and in the Glimm scheme. Still, the following, simple symmetry property for system (1.1) proved in [16], is useful also for the interactions in front tracking.

Lemma 3.3. [16, Lemma 3.1] Under the transformation $x \mapsto-x$, a 1-wave connecting $U_{l}$ to $U_{r}$ becomes a 3-wave connecting $U_{r}$ to $U_{l}$, and vice versa. $A$ 2-wave is unchanged under this transformation, and a non-physical front becomes a nonphysical front traveling in the opposite direction. Furthermore, the leftmost wave with respect to $x$ will become the rightmost wave with respect to $-x$, and so on.

One of our main goals is to show that the approximate solution can be constructed at any time in a finite number of steps. Therefore we look at which interactions increase the number of fronts present. Firstly, recall that the solution of a Riemann problem consists of up to three waves, one from each family. Hence, the solution found by the approximate Riemann solver has four or more fronts if, and only if, a rarefaction wave splits into several fronts. For an interaction between three or more fronts solved by the approximate solver, the number of fronts can therefore only increase due to splitting of rarefaction waves.

Furthermore, an outgoing contact discontinuity is only present if there is an incoming contact discontinuity. Thus, the number of fronts for an interaction between two fronts, none of which are contact discontinuities, can only increase due to splitting of rarefaction waves.

Whenever the simplified solver is used for an interaction between two incoming fronts, we get two outgoing physical waves and one outgoing non-physical front. If there are three or more incoming fronts, the simplified solver gives three outgoing physical waves and one non-physical front. Hence, for an interaction solved by the 
simplified solver, the number of physical fronts can increase only due to splitting of rarefaction waves.

Except for split rarefaction waves, the number of fronts increases only for the interaction between a contact discontinuity and one other front solved by the approximate solver. These interactions have at least three outgoing fronts, and we refer to them as $\gamma$-collisions.

Definition 3.4. A $\gamma$-collision is the interaction between a contact discontinuity and a 1- or 3-front.

The four different $\gamma$-collisions, where symmetry reduces it to two distinct cases, are discussed discussed in the proof of Lemma 3.8 in Section 3.2 .

If the strength of an outgoing rarefaction wave is larger than $\delta$, it splits into several fronts. The interactions where this might happen are either a new rarefactioncollision or an increasing rarefaction-collision as defined below.

Definition 3.5. A new rarefaction-collision is an interaction where there is an outgoing rarefaction wave of a family in which there are no incoming rarefactionfronts.

Definition 3.6. An increasing rarefaction-collision is an interaction where the strength of an outgoing rarefaction wave is greater than the sum of the strengths of the incoming rarefaction-fronts of the same family.

Note that a $\gamma$-collision can also be a new rarefaction-collision, an increasing rarefaction-collision, or even both.

Summing up the front tracking construction, we have defined a piecewise constant function $U^{\delta}$, so that for all fixed $t, U^{\delta}(\cdot, t)$ is a piecewise constant function. Furthermore the construction gives a sequence of collision times $\tau_{1}<\tau_{2}<\ldots$, and $U^{\delta}(\cdot, t)$ is defined for all $t \leq \lim _{n \rightarrow \infty} \tau_{n}$. We shall show that either $\left\{\tau_{n}\right\}$ is a finite sequence or $\lim _{n} \tau_{n}=\infty$, i.e., that $U^{\delta}(\cdot, t)$ can be constructed for any $t>0$.

3.2. The decreasing Glimm functional. Set $t_{n}=\left(\tau_{n}+\tau_{n+1}\right) / 2$, where we have defined $\tau_{0}=0$, and define the functional

$$
G\left(t_{n}\right):=F\left(t_{n}\right)+3 C_{1}(\bar{\gamma}-1) Q_{1}\left(t_{n}\right)+3 C_{2} Q_{2}\left(t_{n}\right),
$$

where $C_{1}$ is the constant appearing in the estimates given by $(3.16)$ for the interaction of Type Bbii, cf. the proof of Lemma 3.8.

$$
C_{2}:=\frac{c_{2}}{\min \left\{r_{\min }^{\prime}, s_{\min }^{\prime}\right\}}=k c_{2},
$$

where $c_{2}$ is the constant from Lemma 2.2 and

$$
k:=\frac{1}{\min \left\{r_{\min }^{\prime}, s_{\min }^{\prime}\right\}} .
$$

Note that both $C_{1}$ and $C_{2}$ are constants only depending on $p_{\min }, p_{\max }$ and $\bar{\gamma}$. This is the same functional as the Glimm functional defined in [16], and the two first terms are similar to the Glimm functional used in [21]. The linear functional $F$ and the two quadratic functionals $Q_{1}$ and $Q_{2}$ are defined by

$$
\begin{aligned}
F\left(t_{n}\right) & :=\sum\left\{|\theta| \mid \text { all shock-fronts } \theta \text { at } t=t_{n}\right\}, \\
Q_{1}\left(t_{n}\right) & :=\sum\left\{|\alpha||\beta| \mid \text { all approaching } 1 \text { - and } 3 \text {-shock-fronts at } t=t_{n}\right\}, \\
Q_{2}\left(t_{n}\right) & :=\sum\left\{|\zeta||\theta| \mid \text { all approaching pairs of } \zeta \text { and } \theta \text { at } t=t_{n}\right\},
\end{aligned}
$$

where two fronts of different families are approaching if the front of the lowest family is to the right of the other. Note that $F$ and $Q_{1}$ only sum over shock-fronts, 
while $Q_{2}$ also sums over rarefaction-fronts. Furthermore, none of the terms involve the strength of non-physical fronts.

We call the lines $t=t_{n}$ time lines. The only difference between the functionals above and the functionals used for the Glimm scheme in [16] is that the above ones are defined on time lines, while the functionals in [16 are defined on mesh curves.

We need two more functionals, one summing over all shock- and rarefactionfronts at $t=t_{n}$ and one summing over the contact discontinuities at $t=t_{n}$. Note that the sum of all contact discontinuities is constant for all time lines. We define

$$
\begin{aligned}
L\left(t_{n}\right) & :=\sum\left\{|\theta| \mid \text { all } \theta \text { at } t=t_{n}\right\}, \\
F_{\gamma} & :=\sum\{|\zeta| \mid \text { all } \zeta\} .
\end{aligned}
$$

We will show that $G$ is a decreasing functional in time. Let

$$
C=\min \{\tilde{C}, 1\},
$$

where the minimum is taken over all the constants $\tilde{C}$ appearing in the estimates for interactions of Type Ba discussed in the proof of Lemma 3.8. Note that $0<C \leq 1$ depends only on $p_{\min }, p_{\max }$ and $\bar{\gamma}$. The rest of this subsection will be devoted to proving the following result:

Proposition 3.7. If

$$
3 C_{1}(\bar{\gamma}-1) L\left(t_{0}\right) \leq \frac{C}{3} \quad \text { and } \quad 3 C_{2} F_{\gamma} \leq \frac{C}{3} .
$$

then $G$ defined by 3.3 is decreasing and $F\left(t_{n}\right) \leq \frac{5}{3} L\left(t_{0}\right)$. In particular, $G$ decreases by at least $\frac{2}{3} q$ across an increasing rarefaction-collision where the strength of the rarefaction wave increases by $q>0$, by at least $\frac{2}{3}\left|\theta^{\prime}\right|$ across a new rarefactioncollision where $\theta^{\prime}$ denotes the new outgoing rarefaction wave, and by at least $3 k\left|\theta^{n p}\right|$ for an interaction where a non-physical front is generated.

We prove this proposition through a series of lemmas where we start by considering interactions between two fronts, then gradually build up to interactions of the general form given by $(3.2)$, including incoming non-physical fronts. For all possible interactions in front tracking we show that $G$ is decreasing and, in particular, we identify all new or increasing rarefaction-collisions and all interactions generating a non-physical front.

Before we state and prove the different lemmas, we present the general idea based on induction on successive time lines: First we show that $G\left(t_{1}\right)-G\left(t_{0}\right) \leq 0$. Then we assume $G\left(t_{n}\right) \leq G\left(t_{n-1}\right) \leq \cdots \leq G\left(t_{0}\right)$. The induction step is to show that $\Delta G:=G\left(t_{n+1}\right)-G\left(t_{n}\right) \leq 0$. Note that if $G$ is decreasing up to $t=t_{n}$, then we have

$$
\begin{aligned}
F\left(t_{n}\right) & \leq G\left(t_{n}\right) \leq \cdots \leq G\left(t_{0}\right)=F\left(t_{0}\right)+3 C_{1}(\bar{\gamma}-1) Q_{1}\left(t_{0}\right)+3 C_{2} Q_{2}\left(t_{0}\right) \\
& \leq F\left(t_{0}\right)+3 C_{1}(\bar{\gamma}-1)\left(F\left(t_{0}\right)\right)^{2}+3 C_{2} L\left(t_{0}\right) F_{\gamma} \\
& \leq\left(1+3 C_{1}(\bar{\gamma}-1) F\left(t_{0}\right)+3 C_{2} F_{\gamma}\right) L\left(t_{0}\right) \\
& \leq\left(1+3 C_{1}(\bar{\gamma}-1) L\left(t_{0}\right)+3 C_{2} F_{\gamma}\right) L\left(t_{0}\right) \\
& \leq\left(1+\frac{C}{3}+\frac{C}{3}\right) L\left(t_{0}\right) \leq \frac{5}{3} L\left(t_{0}\right) .
\end{aligned}
$$

We only give the estimates for $\Delta G$ here. Estimating $G\left(t_{1}\right)-G\left(t_{0}\right)$ is very similar, giving terms involving $F\left(t_{0}\right)$ where the estimate for $\Delta G$ has terms involving $F\left(t_{n}\right)$.

For the more involved interactions we use a computational trick where we divide the interaction into steps where only a part of the fronts interact at each step. It is important to note that in the front tracking algorithm all fronts in an interaction meet at the same point and that no speeds are altered. It is just in the estimation 
of $\Delta G$ we do this step procedure as a formal trick to go from the incoming fronts to a set of fronts which are comparable to the outgoing fronts. Note also that the outgoing fronts are not altered in this process. This method corresponds to the use of inner diamonds for the Glimm scheme in 16. DiPerna [10] constructs the outgoing solution by resolving the interaction into a composition of binary interactions. This method of decomposition is similar to our formal method of dividing an interaction into steps.

Thus, we divide the interaction into $l$ steps where only some of the fronts interact at each step, the rest is left unchanged. As long as the interaction at one step is an interaction already analyzed, we know that $G$ decreases across that step. We continue this until we at some point directly can show that $G$ is decreasing across the last step, where the last step is going from some collection of fronts to the outgoing fronts. Formally, the steps are obtained by shifting the speeds of the incoming fronts slightly, so that only the intended fronts meet at a shifted collision point. This is done for each step and we introduce intermediate time lines, $t=t_{i}^{*}$, so that the interaction at the $i$ th step lies between $t_{i-1}^{*}$ and $t_{i}^{*}$ where $t_{0}^{*}=t_{n}$ and $t_{l}^{*}=t_{n+1}$. As long as we have $\Delta G_{i}:=G\left(t_{i}^{*}\right)-G\left(t_{i-1}^{*}\right) \leq 0$ for $i=1, \ldots, l$, it follows that $\Delta G \leq 0$. Note again that this step procedure is only a computational trick, and that the front-tracking algorithm as such involves no shifting of speeds.

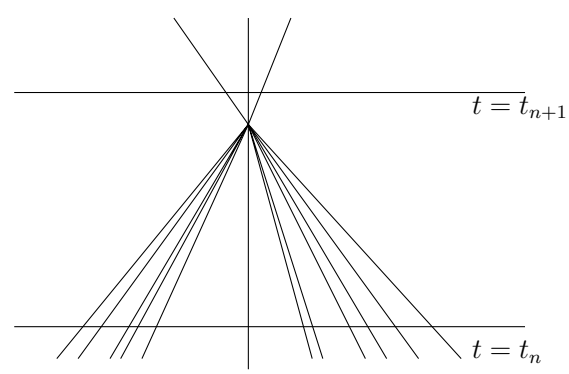

(a) The original interaction.

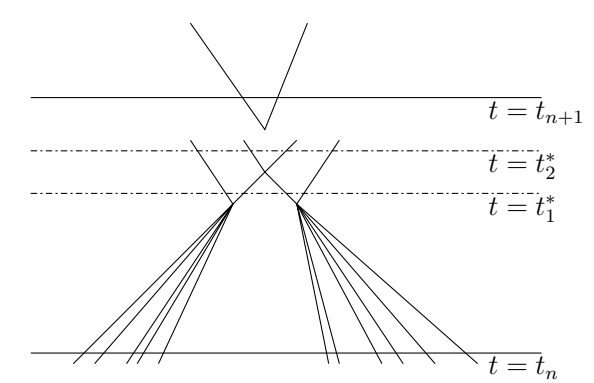

(b) The interaction divided into steps.

Figure 7. A typical interaction of the form $\sum_{i} \eta_{i}+\sum_{j} \epsilon_{j}$.

Figure 7 shows how a typical interaction of the type $\sum_{i} \eta_{i}+\sum_{j} \epsilon_{j}$ is divided into two additional steps. First we let all 3 -fronts interact at one collision point whereas all 1-fronts interact at a different point. Both interactions result in a 1-wave and a 3 -wave. At the second step we let the approaching 3 - and 1-wave interact. Thus, at $t=t_{2}^{*}$ we have a collection of four waves and we compare these to the outgoing fronts. Note that we have not shifted or altered the outgoing fronts at any point in this step procedure.

For some cases we use an additional trick to avoid getting too many steps. Instead of letting some fronts interact at a shifted collision point, we replace the fronts with new fronts connecting the same left and right state. Since this is not a valid interaction, we need to show that $\Delta G_{i} \leq 0$ for this step, and we do that by comparing the new fronts with the replaced fronts. Still this is just a formal trick and the outgoing fronts are not altered.

In Lemma 3.8 through Lemma 3.14 we cover all possible interactions, and we start by the cases with two interacting fronts. Recall that these are the same interactions as for the Glimm scheme, cf. [16], and they are labeled in the same manner as in [16]. 
Lemma 3.8. For all interactions between two fronts we have $\Delta G \leq 0$. In particular, $\Delta G \leq-\frac{2}{3} q$ for all increasing rarefaction-collisions where the strength of the rarefaction wave has increased by $q>0$ and $\Delta G \leq-\frac{2}{3}\left|\theta^{\prime}\right|$ for new rarefactioncollisions where $\theta^{\prime}$ denotes the new rarefaction wave. Moreover, $\Delta G \leq-\frac{1}{9} C_{2}|\zeta||\theta|$ for all $\gamma$-collisions where $\theta$ is the incoming front, and $\Delta G \leq-3 k\left|\theta^{n p}\right|$ for interactions generating a non-physical front.

Proof. The possible interactions between two fronts are the same as the interactions of Type B considered when using the Glimm scheme, cf. [16]. Therefore, we here give the estimates without proofs. All the estimates for interactions without a contact discontinuity are obtained from the estimates by Nishida and Smoller in 21, while the estimates for interactions with a contact discontinuity are found using Lemma 2.2. The estimates for the interactions between a contact discontinuity and another front solved by the simplified solver are also obtained using Lemma 2.2

Type Ba: Two waves of the same family.

(i) $\alpha_{1}+\alpha_{2} \rightarrow \alpha^{\prime}+\nu^{\prime}$, symmetric to $\beta_{1}+\beta_{2} \rightarrow \mu^{\prime}+\beta^{\prime}$ : This is a new rarefactioncollision and we have

$$
\left|\alpha^{\prime}\right|-\left|\alpha_{1}\right|-\left|\alpha_{2}\right|=-\left|\nu^{\prime}\right| \Rightarrow \Delta G \leq-\frac{2}{3}\left|\nu^{\prime}\right| .
$$

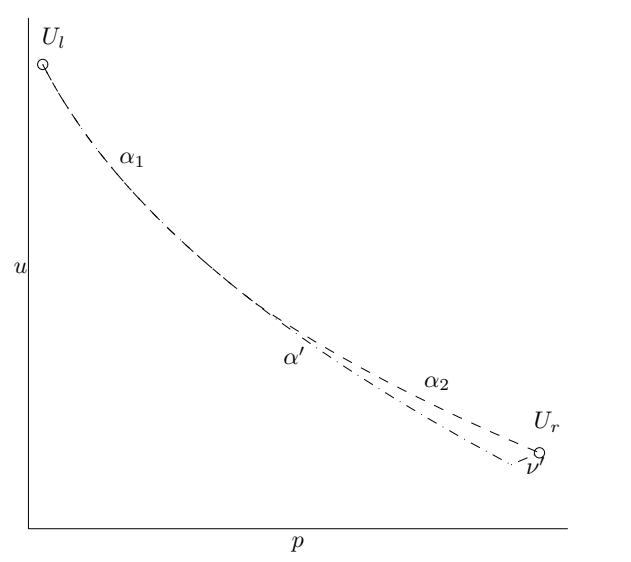

Figure 8. The interaction $\alpha_{1}+\alpha_{2} \rightarrow \alpha^{\prime}+\nu^{\prime}$.

(ii) $\alpha+\mu$, symmetric to $\nu+\beta$. There are two possible outcomes:

- $\alpha+\mu \rightarrow \mu^{\prime}+\beta^{\prime}$ : For this case we have

$$
\left|\mu^{\prime}\right| \leq|\mu|,\left|\beta^{\prime}\right|-|\alpha| \leq-\tilde{C}\left|\beta^{\prime}\right| \Rightarrow \Delta G \leq 0
$$

- $\alpha+\mu \rightarrow \alpha^{\prime}+\beta^{\prime}$ : We have

$$
\left|\alpha^{\prime}\right|+\left|\beta^{\prime}\right|-|\alpha| \leq-\tilde{C}\left|\beta^{\prime}\right| \Rightarrow \Delta G \leq 0 .
$$

(iii) $\mu+\alpha$, symmetric to $\beta+\nu$ : There are two possible outcomes:

- $\mu+\alpha \rightarrow \mu^{\prime}+\beta^{\prime}$ : For this case

$$
\left|\mu^{\prime}\right| \leq|\mu|,\left|\beta^{\prime}\right|-|\alpha| \leq-\tilde{C}\left|\beta^{\prime}\right| \Rightarrow \Delta G \leq 0
$$

- $\mu+\alpha \rightarrow \alpha^{\prime}+\beta^{\prime}$. In this case, the interaction is replaced by a new one,

$$
\mu+\alpha \stackrel{\Delta G_{1}}{\longrightarrow} \bar{\beta}+\bar{\alpha} \stackrel{\Delta G_{2}}{\longrightarrow} \alpha^{\prime}+\beta^{\prime},
$$

for which we have the estimate

$$
|\bar{\alpha}|+|\bar{\beta}|-|\alpha| \leq-\tilde{C}|\bar{\beta}| \Rightarrow \Delta G_{1} \leq 0 .
$$




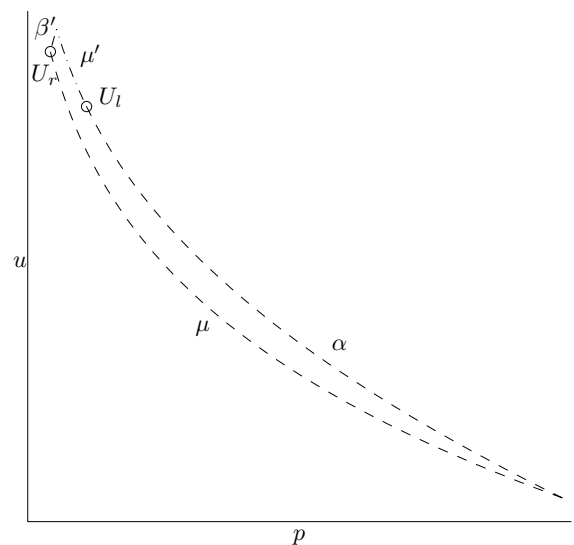

(a) $\alpha+\mu \rightarrow \mu^{\prime}+\beta^{\prime}$.

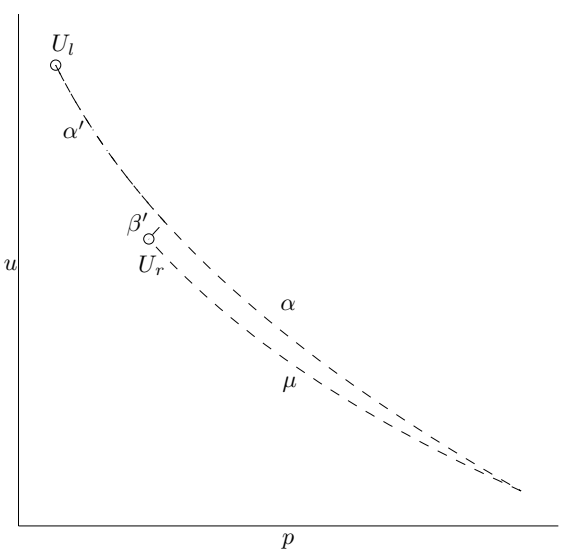

(b) $\alpha+\mu \rightarrow \alpha^{\prime}+\beta^{\prime}$.

FiguRE 9. The interaction $\alpha+\mu$.

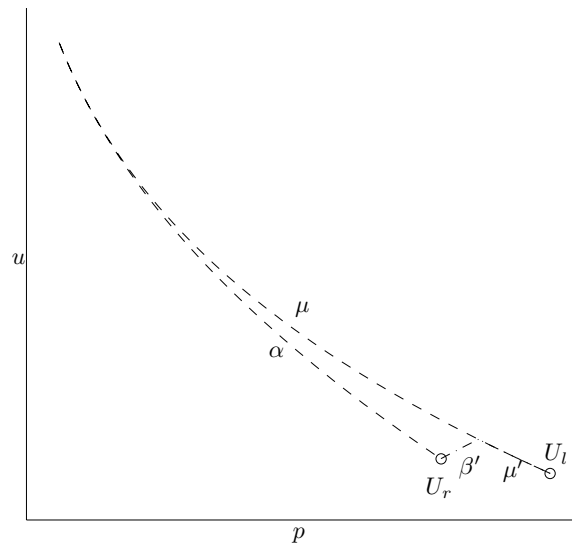

(a) $\mu+\alpha \rightarrow \mu^{\prime}+\beta^{\prime}$.

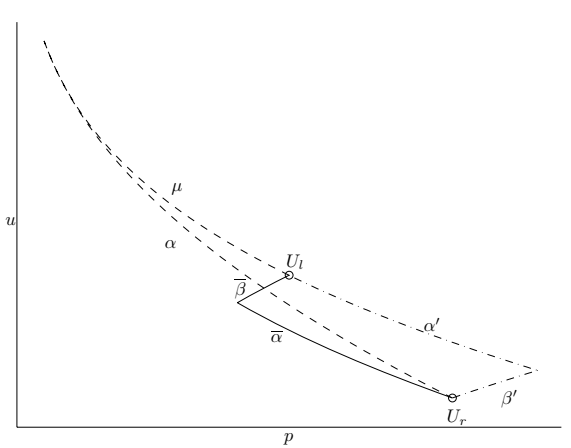

(b) $\mu+\alpha \rightarrow \alpha^{\prime}+\beta^{\prime}$.

FiguRE 10. The interaction $\mu+\alpha$.

Furthermore, we have $\Delta G_{2} \leq 0$ by estimate 3.16 for $\beta+\alpha$ below, cf. Type Bbii. Hence, $\Delta G \leq 0$.

Type Bb: Different families, no contact discontinuity.

(i) $\nu+\mu \rightarrow \nu^{\prime}+\mu^{\prime}$. None of the rarefaction-fronts increase, and we have

$$
\left|\mu^{\prime}\right| \leq|\mu|,\left|\nu^{\prime}\right| \leq|\nu| \Rightarrow \Delta G_{1} \leq 0 \text {. }
$$

(ii) $\beta+\alpha \rightarrow \alpha^{\prime}+\beta^{\prime}$. We have

$$
\left|\alpha^{\prime}\right|-|\alpha| \leq(\bar{\gamma}-1) C_{1}|\alpha||\beta|,\left|\beta^{\prime}\right|-|\beta| \leq(\bar{\gamma}-1) C_{1}|\alpha||\beta|
$$

thus

$$
\Delta G \leq-\frac{1}{9}(\bar{\gamma}-1) C_{1}|\alpha||\beta|
$$

(iii) $\nu+\alpha \rightarrow \alpha^{\prime}+\nu^{\prime}$, symmetric to $\beta+\mu \rightarrow \mu^{\prime}+\beta^{\prime}$. This is an increasing rarefaction-collision where we for $q>0$ have

$$
\left|\alpha^{\prime}\right|-|\alpha|=-q,\left|\nu^{\prime}\right|-|\nu|=q \Rightarrow \Delta G \leq-\frac{2}{3} q .
$$




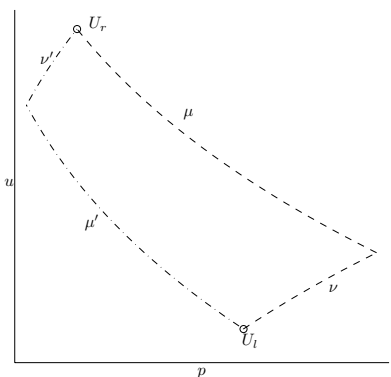

(a) $\nu+\mu \rightarrow \mu^{\prime}+\nu^{\prime}$.

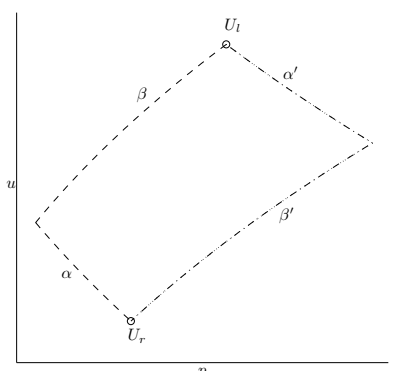

(b) $\beta+\alpha \rightarrow \alpha^{\prime}+\beta^{\prime}$.

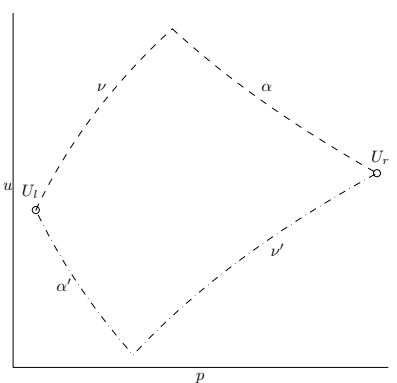

(c) $\nu+\alpha \rightarrow \alpha^{\prime}+\nu^{\prime}$.

Figure 11. The interactions of Type Bb

Type Bc: With a contact discontinuity: These are the four possible $\gamma$-collisions.

(i) $\zeta+\mu$, symmetric to $\nu+\zeta$ : There are two possible outcomes for this $\gamma$-collision, and, in addition, we have the case where the simplified Riemann solver is used, introducing a non-physical front.

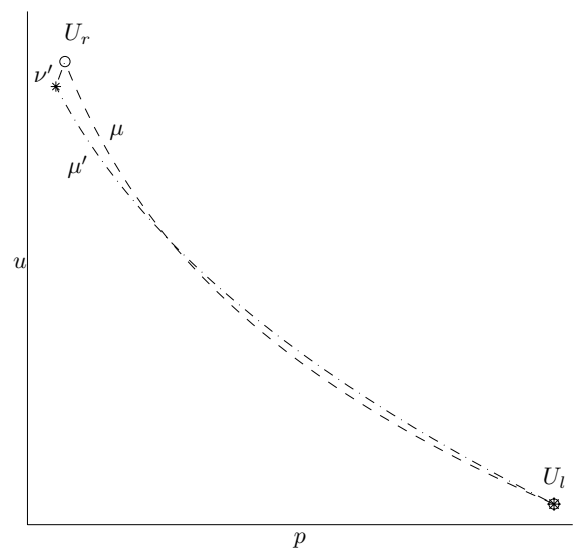

(a) $\zeta+\mu \rightarrow \mu^{\prime}+\zeta+\nu^{\prime}$.

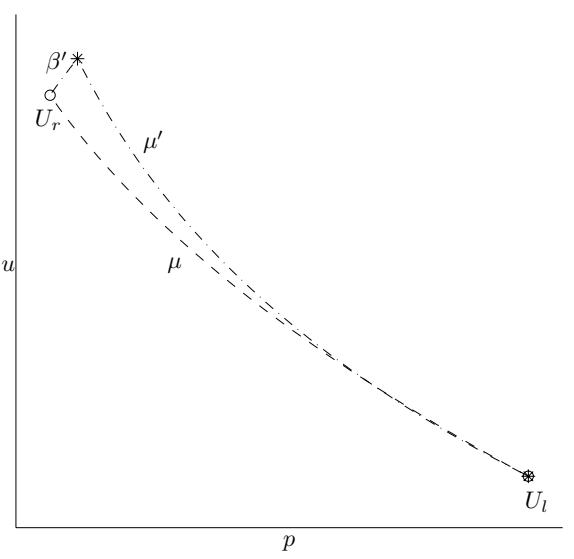

(b) $\zeta+\mu \rightarrow \mu^{\prime}+\zeta+\beta^{\prime}$.

FiguRE 12. The interaction $\zeta+\mu$.

- $\zeta+\mu \rightarrow \mu^{\prime}+\zeta+\nu^{\prime}$ : This interaction is a new rarefaction-collision and an increasing rarefaction-collision with $q=\left|\nu^{\prime}\right|$. We have

$$
\left|\mu^{\prime}\right|-|\mu|=\left|\nu^{\prime}\right| \leq C_{2}|\mu||\zeta|,
$$

from which we find

$$
\Delta G \leq-\frac{8}{3} C_{2}|\mu||\zeta| \leq-\frac{2}{3}\left|\nu^{\prime}\right|-\frac{2}{3} q .
$$

- $\zeta+\mu \rightarrow \mu^{\prime}+\zeta+\beta^{\prime}$ : The rarefaction-front does not increase and $\left|\mu^{\prime}\right|-|\mu| \leq 0,\left|\beta^{\prime}\right| \leq C_{2}|\mu||\zeta| \Rightarrow \Delta G \leq-\frac{10}{9} C_{2}|\mu||\zeta|$.

- $\zeta+\mu \rightarrow \mu^{\prime}+\zeta+\theta^{\text {np }}$ : By construction, $\left|\mu^{\prime}\right|=|\mu|$. Using Lemma 2.2 we find

$$
\left|\theta^{\mathrm{np}}\right| \leq c_{2}|\mu||\zeta|
$$

from which we get

$$
\Delta G \leq-3 C_{2}|\mu||\zeta| \leq-3 k\left|\theta^{\mathrm{np}}\right|,
$$


where $k$ given by 3.5 depends only on $p_{\min }, p_{\max }$ and $\bar{\gamma}$.

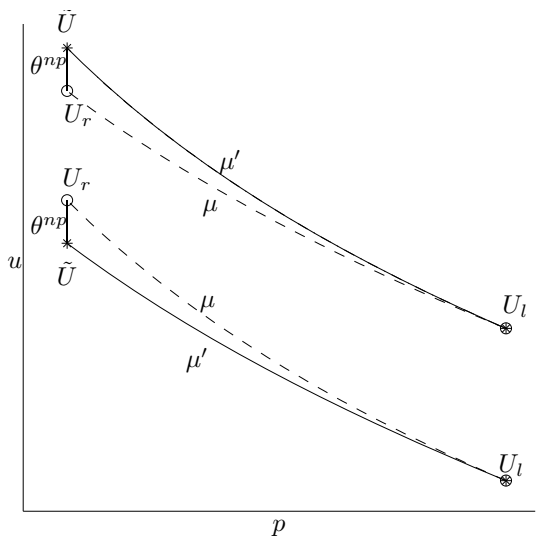

(a) $\zeta+\mu \rightarrow \mu^{\prime}+\zeta+\theta^{\text {np }}$.

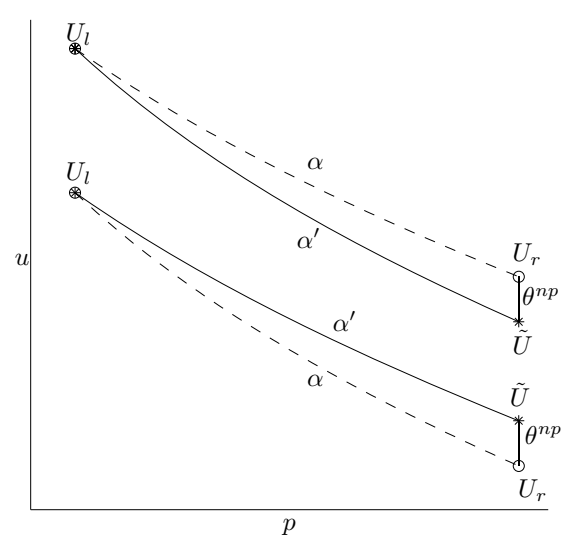

(b) $\zeta+\alpha \rightarrow \alpha^{\prime}+\zeta+\theta^{\text {np }}$.

FIGURE 13. The interaction $\zeta+\epsilon$ solved using the simplified solver.

(ii) $\zeta+\alpha$, symmetric to $\beta+\zeta$. This $\gamma$-collision has two possible outcomes, in addition to the case with a non-physical front.

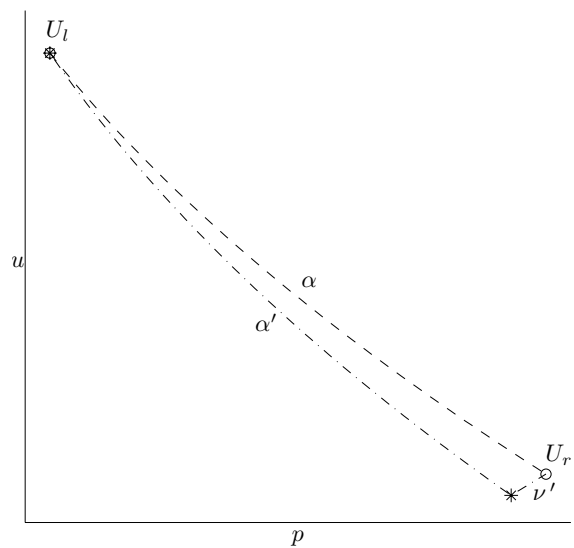

(a) $\zeta+\alpha \rightarrow \alpha^{\prime}+\zeta+\nu^{\prime}$.

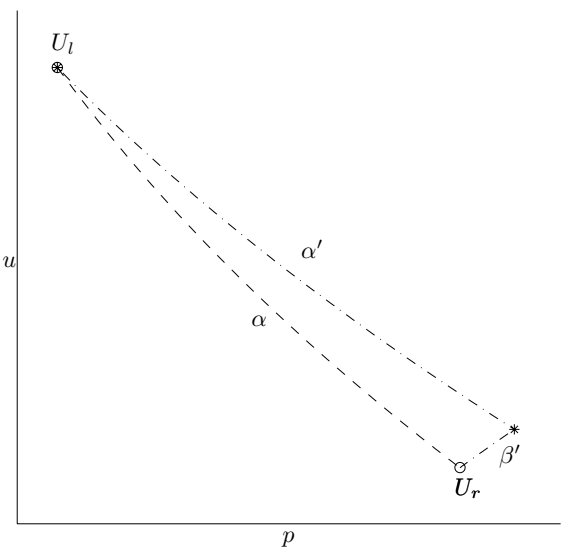

(b) $\zeta+\alpha \rightarrow \alpha^{\prime}+\zeta+\beta^{\prime}$.

FiguRE 14. The interaction $\zeta+\alpha$.

- $\zeta+\alpha \rightarrow \alpha^{\prime}+\zeta+\nu^{\prime}$ : For this new rarefaction-collision we have

$$
\left|\alpha^{\prime}\right|-|\alpha| \leq 0,\left|\nu^{\prime}\right| \leq C_{2}|\alpha||\zeta|,
$$

thus,

$$
\Delta G \leq-\frac{2}{3} C_{2}|\alpha||\zeta| \leq-\frac{2}{3}\left|\nu^{\prime}\right| .
$$

- $\zeta+\alpha \rightarrow \alpha^{\prime}+\zeta+\beta^{\prime}$ : For this case we have

$$
\left|\alpha^{\prime}\right|-|\alpha|=\left|\beta^{\prime}\right| \leq C_{2}|\alpha||\zeta| \Rightarrow \Delta G \leq-\frac{1}{9} C_{2}|\zeta||\beta| .
$$

- $\zeta+\alpha \rightarrow \alpha^{\prime}+\zeta+\theta^{\text {np }}$ : By construction, $\left|\alpha^{\prime}\right|=|\alpha|$ and by Lemma 2.2

$$
\left|\theta^{\mathrm{np}}\right| \leq c_{2}|\mu||\zeta|
$$


Thus,

$$
\Delta G \leq-3 C_{2}|\alpha||\zeta| \leq-3 k\left|\theta^{\text {np }}\right|,
$$

where $k$ only depends on $p_{\min }, p_{\max }$ and $\bar{\gamma}$.

With the basic interactions between two fronts covered, we are able to consider more involved interactions. First interactions between arbitrary many fronts of the same family are studied. Two interactions of this kind are given in Figure 15, see also Example 3.10 below. Note that no interaction of this form can be an increasing rarefaction-collision.

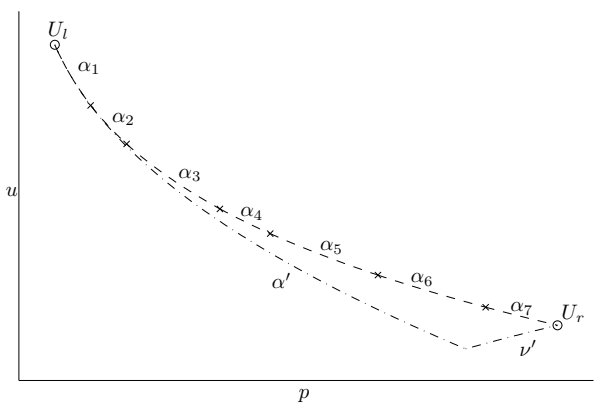

(a) $\sum_{i=1}^{7} \alpha_{i} \rightarrow \alpha^{\prime}+\nu^{\prime}$.

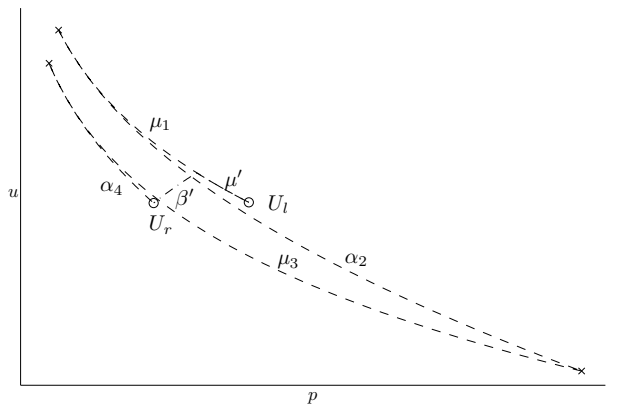

(b) $\mu_{1}+\alpha_{2}+\mu_{3}+\alpha_{4} \rightarrow \mu^{\prime}+\beta^{\prime}$.

Figure 15. Some interactions of the form (3.19).

Lemma 3.9. For all interactions between arbitrary many fronts of the same family where two adjacent fronts cannot both be rarefaction-fronts, we have $\Delta G \leq 0$, and in particular, $\Delta G \leq-\frac{2}{3}\left|\theta^{\prime}\right|$ for new rarefaction-collisions where $\theta^{\prime}$ denotes the new rarefaction wave. Furthermore, there are three possible outcomes for these interactions;

$$
\sum_{i=1}^{n} \epsilon_{i} \rightarrow\left\{\begin{array} { l } 
{ \mu ^ { \prime } + \beta ^ { \prime } , } \\
{ \alpha ^ { \prime } + \nu ^ { \prime } , } \\
{ \alpha ^ { \prime } + \beta ^ { \prime } , }
\end{array} \quad \text { symmetric to } \sum _ { i = 1 } ^ { n } \eta _ { i } \rightarrow \left\{\begin{array}{l}
\alpha^{\prime}+\nu^{\prime}, \\
\mu^{\prime}+\beta^{\prime}, \\
\alpha^{\prime}+\beta^{\prime} .
\end{array}\right.\right.
$$

Proof. We prove the lemma for interactions between three or more 1-fronts, the interactions with $n=2$ are already covered by Lemma 3.8. None of the interactions can have two rarefaction waves as outgoing waves due to property (i) of Lemma 2.1 Recall also that $p$ increases along a 1-shock wave and decreases along a 1-rarefaction wave.

Consider first the case $\alpha^{\prime}+\nu^{\prime}$. Then the interaction is a new rarefaction-collision where $U_{r}$ is to the right of $U_{l}$ and above the 1 -shock wave starting at $U_{l}$. Since only the $\alpha_{i}$-fronts among the incoming fronts bring us to the right, we have

$$
\left|\alpha^{\prime}\right|-\sum_{i=1}^{n}\left|\alpha_{i}\right| \leq-\left|\nu^{\prime}\right| \Rightarrow \Delta G \leq-\frac{2}{3}\left|\nu^{\prime}\right| .
$$

For the case $\mu^{\prime}+\beta^{\prime}, U_{r}$ is to the left of $U_{l}$. The only incoming fronts bringing us to the left are the $\mu_{i}$-fronts, thus

$$
\left|\mu^{\prime}\right| \leq \sum_{i=1}^{n}\left|\mu_{i}\right|
$$


Hence, no interaction between fronts of the same family is an increasing rarefactioncollision.

Therefore we consider the last two cases together, that is, $\epsilon^{\prime}+\beta^{\prime}$ where $\epsilon^{\prime}$ is either a shock or a rarefaction wave. We divide the interaction into several steps where two fronts interact at each step, hence, $\Delta G_{j} \leq 0$ by Lemma 3.8. Recall that two adjacent fronts in the interaction cannot both be rarefaction-fronts. The strategy is as follows: Start with the rightmost front and search for the first place where two adjacent fronts are of different types, i.e., $\alpha_{i}+\mu_{i+1}$ or $\mu_{i}+\alpha_{i+1}$. Let these fronts interact with outcome $\tilde{\epsilon}_{k}+\tilde{\beta}_{k}$. Whenever there is a 1-shock to the right of $\tilde{\beta}_{k}$, we proceed by letting them interact; $\tilde{\beta}_{k}+\alpha \rightarrow \tilde{\alpha}_{k+1}+\tilde{\beta}_{k+1}$, and we repeat this as long as there is a 1 -shock to the right of the 3 -shock. Thus, we end up with a collection of $\beta$-waves as the rightmost waves. Furthermore, whenever this process results in two adjacent rarefaction waves, we recall from property (vii) of Lemma 2.1 that rarefaction waves (and fronts) are additive and we add them up to a new rarefaction wave. We continue this process until all 1-fronts of different types have interacted, and we are left with either $\tilde{\mu}+\sum \tilde{\beta}_{k}$ or $\sum \tilde{\alpha}_{k}+\sum \tilde{\beta}_{k}$. For the first case we have

$$
\sum_{i=1}^{n} \epsilon_{i} \stackrel{\Delta G_{1}}{\longrightarrow} \tilde{\mu}+\sum_{k} \tilde{\beta}_{k} \stackrel{\Delta G_{2}}{\longrightarrow} \mu^{\prime}+\beta^{\prime},
$$

where we already know that $\Delta G_{1} \leq 0$. By property (ix) of Lemma 2.1 it follows that $\sum_{k}\left|\tilde{\beta}_{k}\right|>\left|\beta^{\prime}\right|$, thus, there is a $q>0$ so that

$$
\left|\mu^{\prime}\right|-|\tilde{\mu}|=q,\left|\beta^{\prime}\right|-\sum_{k}\left|\tilde{\beta}_{k}\right|=-q \Rightarrow \Delta G_{2} \leq 0 .
$$

For the latter case we have

$$
\sum_{i=1}^{n} \epsilon_{i} \stackrel{\Delta G_{1}}{\longrightarrow} \sum_{k} \tilde{\alpha}_{k}+\sum_{k} \tilde{\beta}_{k} \stackrel{\Delta G_{2}}{\longrightarrow} \alpha^{\prime}+\beta^{\prime},
$$

where we already know that $\Delta G_{1} \leq 0$. Furthermore, it follows from the properties viii) and (ix) of Lemma 2.1 that

$$
\left|\alpha^{\prime}\right|-\sum_{k}\left|\tilde{\alpha}_{k}\right| \leq 0,\left|\beta^{\prime}\right|-\sum_{k}\left|\tilde{\beta}_{k}\right| \leq 0 \Rightarrow \Delta G_{2} \leq 0 .
$$

This proves the lemma for the interaction $\sum_{i} \epsilon_{i}$, and the results for $\sum_{i} \eta_{i}$ follows by symmetry. However, we include another estimate for the last case discussed above, which will prove useful later. The number of $\tilde{\alpha}_{k}$-fronts is less than or equal to the number of incoming $\alpha_{i}$-fronts. Going carefully through each steps, we find that each $\tilde{\alpha}_{k}$ has a corresponding incoming $\alpha_{i}$ so that

$$
\left|\tilde{\alpha}_{k}\right| \leq \prod_{j \neq i}\left(1+C_{1}(\bar{\gamma}-1)\left|\epsilon_{j}\right|\right)\left|\alpha_{i}\right| \leq \frac{4}{3}\left|\alpha_{i}\right|,
$$

because

$$
\begin{aligned}
\prod_{j \neq i}\left(1+C_{1}(\bar{\gamma}-1)\left|\epsilon_{j}\right|\right) & \leq 1+\frac{3}{2} \sum_{j} C_{1}(\bar{\gamma}-1)\left|\epsilon_{j}\right| \\
& \leq 1+\frac{3}{2} C_{1}(\bar{\gamma}-1) F\left(t_{n-1}\right) \\
& \leq 1+\frac{3}{2} C_{1}(\bar{\gamma}-1) \frac{5}{3} L\left(t_{0}\right) \leq \frac{4}{3}
\end{aligned}
$$


Here we have used that

$$
\prod_{i}\left(1+x_{i}\right) \leq \exp \left(\sum_{i} x_{i}\right) \leq 1+\frac{3}{2} \sum_{i} x_{i}, \quad \text { for } \quad \sum_{i} x_{i} \leq \frac{1}{2} .
$$

Before we continue to the more complicated interactions, we give an example to illustrate how we divide an interaction of the form 3.19 into smaller steps.

Example 3.10. Consider the interaction $\mu_{1}+\alpha_{2}+\mu_{3}+\alpha_{4} \rightarrow \alpha^{\prime}+\beta^{\prime}$ as depicted in Figure 16(a). Dividing this interaction according to the strategy we discussed in the previous proof, see Figure 16(b), we get

$$
\begin{aligned}
\mu_{1}+\alpha_{2}+\left[\mu_{3}+\alpha_{4}\right] \stackrel{\Delta G_{1}}{\longrightarrow}\left[\mu_{1}+\alpha_{2}\right]+\tilde{\alpha}_{1}+\tilde{\beta}_{1} \stackrel{\Delta G_{2}}{\longrightarrow} \tilde{\alpha}_{2}+\left[\tilde{\beta}_{2}+\tilde{\alpha}_{1}\right]+\tilde{\beta}_{1} \\
\stackrel{\Delta G_{3}}{\longrightarrow} \tilde{\alpha}_{2}+\tilde{\alpha}_{3}+\tilde{\beta}_{3}+\tilde{\beta}_{1} \stackrel{\Delta G_{4}}{\longrightarrow} \alpha^{\prime}+\beta^{\prime},
\end{aligned}
$$

where $\Delta G_{i} \leq 0, i=1,2,3$, by Lemma 3.8 . By the properties of shock waves, we have

$$
\left|\alpha^{\prime}\right|-\left|\tilde{\alpha}_{2}\right|-\left|\tilde{\alpha}_{3}\right| \leq 0, \quad\left|\beta^{\prime}\right|-\left|\tilde{\beta}_{1}\right|-\left|\tilde{\beta}_{3}\right| \leq 0, \quad \Rightarrow \quad \Delta G_{4} \leq 0 .
$$

Note that $\mu+\alpha$ as above is the second case of the interaction of Type Baiii discussed in Lemma 3.8, where we introduced an extra step to solve it. This is given in (3.14) where $|\bar{\beta}| \leq|\mu|$ and $|\bar{\alpha}| \leq|\alpha|$. Using this, and the estimates given by (3.16) for $\bar{\beta}+\bar{\alpha}$, we find

$$
\begin{aligned}
\left|\tilde{\alpha}_{2}\right| & \leq\left(1+(\bar{\gamma}-1) C_{1}\left|\bar{\beta}_{2}\right|\right)\left|\bar{\alpha}_{2}\right| \leq\left(1+(\bar{\gamma}-1) C_{1}\left|\mu_{1}\right|\right)\left|\alpha_{2}\right|, \\
\left|\tilde{\alpha}_{3}\right| & \leq\left(1+(\bar{\gamma}-1) C_{1}\left|\tilde{\beta}_{2}\right|\right)\left|\tilde{\alpha}_{1}\right| \\
& \leq\left(1+(\bar{\gamma}-1) C_{1}\left(1+(\bar{\gamma}-1) C_{1}\left|\bar{\alpha}_{2}\right|\right)\left|\bar{\beta}_{2}\right|\right)\left(1+(\bar{\gamma}-1) C_{1}\left|\bar{\beta}_{1}\right|\right)\left|\bar{\alpha}_{2}\right| \\
& \leq\left(1+(\bar{\gamma}-1) C_{1}\left|\mu_{1}\right|\right)\left(1+(\bar{\gamma}-1) C_{1}\left|\alpha_{2}\right|\right)\left(1+(\bar{\gamma}-1) C_{1}\left|\mu_{3}\right|\right)\left|\alpha_{4}\right|,
\end{aligned}
$$

showing that estimate 3.21 holds for $\tilde{\alpha}_{2}$ and $\tilde{\alpha}_{3}$.

Using Lemma 3.9 we are now able to divide the more involved interactions into smaller steps and through this show that $G$ decreases. We start by adding a contact discontinuity to the interactions.

Lemma 3.11. The functional $G$ decreases for all interactions of the form

$$
\zeta+\sum_{i=1}^{n} \epsilon_{i} \text { and the symmetric form } \sum_{i=1}^{n} \eta_{i}+\zeta .
$$

Furthermore, $\Delta G \leq-\frac{2}{3}\left|\theta^{\prime}\right|$ for new rarefaction-collisions where $\theta^{\prime}$ is the new wave, $\Delta G \leq-\frac{2}{3} q$ for increasing rarefaction-collisions where the strength of the rarefaction wave increases by $q>0$, and $\Delta G \leq-3 k\left|\theta^{n p}\right|$ for interactions generating a nonphysical front.

Proof. Let us first consider when $|\zeta| \sum_{i}|\epsilon|>\rho$, so that the approximate solver is used. We then divide interaction $\zeta+\sum_{i=1}^{n} \epsilon_{i}$ into two steps where we let $\sum_{i=1}^{n} \epsilon_{i}$ interact at the first step. By Lemma 3.9 we know that $G$ is decreasing for this interaction and that there are three possible cases. We write this

$$
\zeta+\sum_{i=1}^{n} \epsilon_{i} \stackrel{\Delta G_{1}}{\longrightarrow} \zeta+\left\{\begin{array}{l}
\mu+\beta \\
\alpha+\nu \\
\alpha+\beta
\end{array} \quad \stackrel{\Delta G_{2}}{\longrightarrow} \epsilon^{\prime}+\zeta+\eta^{\prime},\right.
$$

where $\Delta G_{1} \leq 0$. At the second step there are three possible interactions of the form $\zeta+\epsilon+\eta \rightarrow \epsilon^{\prime}+\zeta+\eta^{\prime}$. The outgoing 1-wave is of the same type as the incoming 


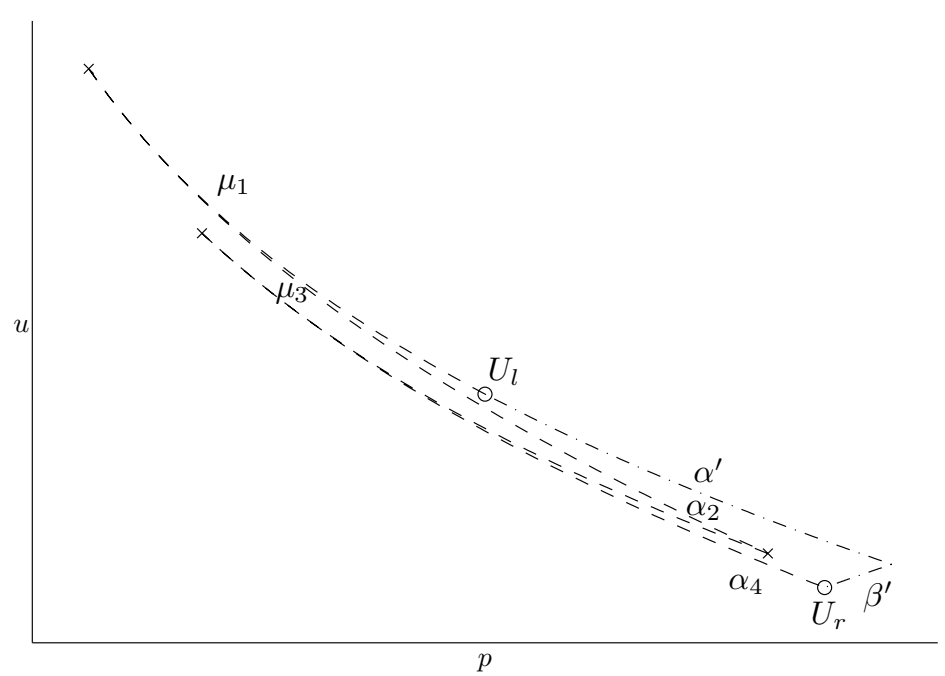

(a) The original interaction

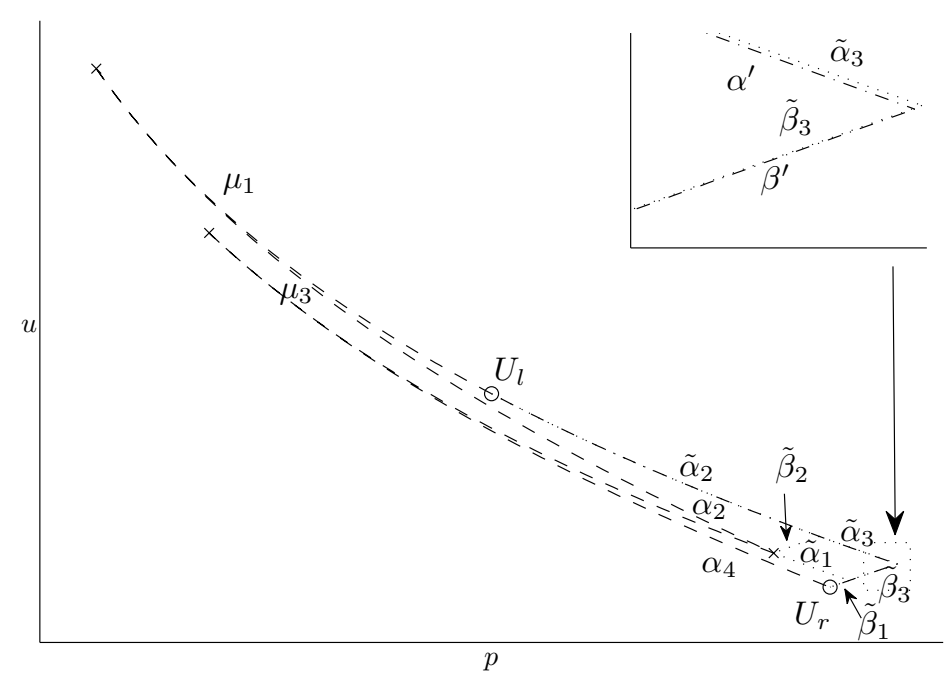

(b) Divided into smaller steps

FiguRE 16. The interaction $\mu_{1}+\alpha_{2}+\mu_{3}+\alpha_{4} \rightarrow \alpha^{\prime}+\beta^{\prime}$ of Example 3.10

1-wave for all of these interactions, while the type of the 3 -wave depends on the different $\gamma$-values, giving two possible cases for each interaction.

Consider first the case where the incoming and outgoing 3 -waves are of the same type. If the intersection between $\epsilon^{\prime}$ and $\eta^{\prime}$ is below the intersection between $\epsilon$ and $\eta$ when viewed in the $(p, u)$-plane, we have

$$
\begin{array}{cl}
\left|\mu^{\prime}\right|-|\mu|=q,\left|\beta^{\prime}\right|-|\beta|=-q & \Rightarrow \Delta G_{2} \leq-\frac{2}{3}\left|\mu^{\prime}\right|, \\
\left|\alpha^{\prime}\right|-|\alpha|=-q,\left|\nu^{\prime}\right|-|\nu|=q & \Rightarrow \Delta G_{2} \leq-\frac{2}{3}\left|\nu^{\prime}\right|, \\
\left|\alpha^{\prime}\right|-|\alpha| \leq 0,\left|\beta^{\prime}\right|-|\beta| \leq 0 & \Rightarrow \Delta G_{2} \leq 0,
\end{array}
$$

for the three interactions, respectively. The two first are increasing rarefactioncollisions, however, the overall interactions are not increasing rarefaction-collisions because property (ii) of Lemma 2.1 yields that $\left|\epsilon^{\prime}\right|$ is less than the sum of the 
strengths of the incoming 1-fronts of the same type. If the intersection in the $(p, u)$-plane between $\epsilon^{\prime}$ and $\eta^{\prime}$ is above the intersection between $\epsilon$ and $\eta$, we apply Lemma 2.2 to $\epsilon$ and $\epsilon^{\prime}$ and find that

$$
\begin{array}{r}
0 \leq\left|\beta^{\prime}\right|-|\beta| \leq|\mu|-\left|\mu^{\prime}\right| \leq C_{2}\left|\mu^{\prime}\right||\zeta| \leq C_{2}|\mu||\zeta| \Rightarrow \Delta G_{2} \leq 0 \\
\left|\alpha^{\prime}\right|-|\alpha| \leq C_{2}|\alpha||\zeta|, \quad\left|\nu^{\prime}\right|-|\nu| \leq 0 \Rightarrow \Delta G_{2} \leq 0 \\
\left|\beta^{\prime}\right|-|\beta|=\left|\alpha^{\prime}\right|-|\alpha| \leq C_{2}|\alpha||\zeta| \Rightarrow \Delta G_{2} \leq 0
\end{array}
$$

for the three interactions, respectively.

Consider now the case where the 3 -waves are of different types. The interactions with $\eta^{\prime}=\nu^{\prime}$ are new rarefaction-collisions, and the interaction with $\epsilon^{\prime}=\mu^{\prime}$ is also an increasing rarefaction-collision with $\left|\nu^{\prime}\right| \leq\left|\mu^{\prime}\right|-|\mu|=q$. We obtain the following estimates;

$$
\begin{gathered}
\left|\nu^{\prime}\right| \leq q=\left|\mu^{\prime}\right|-|\mu| \leq C_{2}|\mu||\zeta| \Rightarrow \Delta G_{2} \leq-\frac{2}{3}\left|\nu^{\prime}\right|-\frac{2}{3} q, \\
\left|\beta^{\prime}\right|-|\beta|=\left|\alpha^{\prime}\right|-|\alpha| \leq C_{2}|\alpha||\zeta| \Rightarrow \Delta G_{2} \leq 0, \\
\left|\alpha^{\prime}\right|-|\alpha|=-\left|\nu^{\prime}\right|-|\beta| \leq-\left|\nu^{\prime}\right| \Rightarrow \Delta G_{2} \leq-\frac{2}{3}\left|\nu^{\prime}\right|,
\end{gathered}
$$

where we used Lemma 2.2 on $\epsilon$ and $\epsilon^{\prime}$ for the two first interactions.

Next, we consider the case where the simplified solver is used to solve $\zeta+\sum_{j=1}^{n} \epsilon_{j}$ with $n \geq 2$. The construction of the solution, as described in Section 3.1. can be viewed as dividing the interaction into three steps. First we let $\sum_{i} \epsilon_{i}$ interact, resulting in $\bar{\epsilon}+\bar{\eta}$, then we solve the interaction between $\zeta+\bar{\epsilon}$ using the simplified solver. Finally, the non-physical front interact with $\bar{\eta}$. Since the non-physical front just passes through without changing its strength, $\eta^{\prime}$ is just $\bar{\eta}$ shifted in the $u$-direction. We write this

$$
\zeta+\sum_{j=1}^{n} \epsilon_{j} \stackrel{\Delta G_{1}}{\longrightarrow}\left\{\begin{array}{l}
{[\zeta+\bar{\mu}]+\bar{\beta} \stackrel{\Delta G_{2}}{\longrightarrow} \mu^{\prime}+\zeta+\left[\theta^{\mathrm{np}}+\bar{\beta}\right] \stackrel{\Delta G_{3}}{\longrightarrow} \mu^{\prime}+\zeta+\beta^{\prime}+\theta^{\mathrm{np}},} \\
{[\zeta+\bar{\alpha}]+\bar{\nu} \stackrel{\Delta G_{2}}{\longrightarrow} \alpha^{\prime}+\zeta+\left[\theta^{\mathrm{np}}+\bar{\nu}\right] \stackrel{\Delta G_{3}}{\longrightarrow} \alpha^{\prime}+\zeta+\nu^{\prime}+\theta^{\mathrm{np}},} \\
{[\zeta+\bar{\alpha}]+\bar{\beta} \stackrel{\Delta G_{2}}{\longrightarrow} \alpha^{\prime}+\zeta+\left[\theta^{\mathrm{np}}+\bar{\beta}\right] \stackrel{\Delta G_{3}}{\longrightarrow} \alpha^{\prime}+\zeta+\beta^{\prime}+\theta^{\mathrm{np}} .}
\end{array}\right.
$$

Figure 6 shows the intermediate fronts for the second interaction, in addition to the solution using the approximate solver. For the first step we have $\Delta G_{1} \leq 0$ by Lemma 3.9 and, from the proof of the lemma, we have

$$
|\bar{\mu}| \leq \sum_{i}\left|\mu_{i}\right|,|\bar{\alpha}| \leq \sum_{i}\left|\alpha_{i}\right|,|\bar{\alpha}| \leq \sum_{k}\left|\tilde{\alpha}_{k}\right| \leq \frac{4}{3} \sum_{i}\left|\alpha_{i}\right|,
$$

respectively, where the last inequality follows from estimate (3.21). For the second step, we have from the proof of Lemma 3.8 and the above estimate that

$$
\left|\theta^{\mathrm{np}}\right| \leq c_{2}|\bar{\epsilon}||\zeta| \leq \frac{4}{3} c_{2}|\zeta| \sum_{i}\left|\epsilon_{i}\right| \text {, and } \Delta G_{2} \leq-3 k\left|\theta^{\mathrm{np}}\right| .
$$

Finally, since $|\bar{\eta}|=\left|\eta^{\prime}\right|$, it follows that $\Delta G_{3}=0$. Hence, $\Delta G \leq \Delta G_{1}+\Delta G_{2}+$ $\Delta G_{3} \leq-3 k\left|\theta^{\text {np }}\right|$, and we have covered the case where non-physical fronts are generated.

Thus, $G$ decreases for all interactions of the form $\zeta+\sum_{j=1}^{n} \epsilon_{j}$ where the case $n=1$ is covered by Lemma 3.8 . The result for $\sum_{i=1}^{n} \eta_{i}+\zeta$ follows by symmetry.

Next, we consider interactions between arbitrary many fronts of the first and third family. 
Lemma 3.12. The functional $G$ is decreasing for all interactions of the form

$$
\sum_{i=1}^{n} \eta_{i}+\sum_{j=1}^{m} \epsilon_{j} .
$$

Furthermore, $\Delta G \leq-\frac{2}{3} q$ for increasing rarefaction-collisions where $q>0$ is the increase in the strength of the rarefaction wave, and $\Delta G \leq-\frac{2}{3}\left|\theta^{\prime}\right|$ for new rarefaction-collisions where $\theta^{\prime}$ is the new rarefaction wave.

Proof. If $n>1$ and $m>1$, we divide these interactions into steps as follows;

$$
\sum_{i=1}^{n} \eta_{i}+\sum_{j=1}^{m} \epsilon_{j} \stackrel{\Delta G_{1}}{\longrightarrow}\left\{\begin{array}{l}
\mu_{1}+\beta_{1} \\
\alpha_{1}+\nu_{1} \\
\alpha_{1}+\beta_{1}
\end{array}+\left\{\begin{array}{l}
\mu_{2}+\beta_{2} \\
\alpha_{2}+\nu_{2} \\
\alpha_{2}+\beta_{2}
\end{array} \quad \stackrel{\Delta G_{n}}{\longrightarrow} \epsilon^{\prime}+\eta^{\prime}\right.\right.
$$

where $\Delta G_{1} \leq 0$ by Lemma 3.9 . There are nine possible interactions at the third step, but three of these are symmetric to one of the other, leaving us with six interactions to consider. If $n=1$ and $m>1$ (symmetric to $n>1$ and $m=1$ ), the interactions are divided as follows;

$$
\eta+\sum_{j=1}^{n} \epsilon_{j} \stackrel{\Delta G_{1}}{\longrightarrow}\left\{\begin{array}{l}
\beta_{1} \\
\nu_{1}
\end{array}+\left\{\begin{array}{l}
\mu_{2}+\beta_{2} \\
\alpha_{2}+\nu_{2} \\
\alpha_{2}+\beta_{2}
\end{array} \quad \stackrel{\Delta G_{n}}{\longrightarrow} \epsilon^{\prime}+\eta^{\prime},\right.\right.
$$

where $\Delta G_{1} \leq 0$ by Lemma 3.9 . This gives us six interactions to consider for the second step. The interactions with $n=m=1$ are already covered by Lemma 3.8.

We consider first the interactions with only one combination of outgoing waves, that is,

$$
\alpha_{1}+\beta_{1}+\alpha_{2}+\beta_{2} \rightarrow \alpha^{\prime}+\beta^{\prime} \text { and } \mu_{1}+\beta_{1}+\mu_{2}+\beta_{2} \rightarrow \mu^{\prime}+\beta^{\prime},
$$

where the last one is symmetric to $\alpha_{1}+\nu_{1}+\alpha_{2}+\nu_{2} \rightarrow \alpha^{\prime}+\nu^{\prime}$. We divide these into one extra step;

$$
\epsilon_{1}+\left[\beta_{1}+\epsilon_{2}\right]+\beta_{2} \stackrel{\Delta G_{2}}{\longrightarrow} \epsilon_{1}+\bar{\epsilon}+\bar{\beta}+\beta_{2} \stackrel{\Delta G_{3}}{\longrightarrow} \epsilon^{\prime}+\beta^{\prime},
$$

where all $\epsilon$-fronts are of the same type. From Lemma 3.8 we have the necessary estimate on $\Delta G_{2}$. For the interactions at the last step, the latter one being an increasing rarefaction-collision, we obtain from the properties of the shock waves that

$$
\begin{gathered}
\left|\alpha^{\prime}\right|-\left|\alpha_{1}\right|-|\bar{\alpha}| \leq 0,\left|\beta^{\prime}\right|-\left|\beta_{2}\right|-|\bar{\beta}| \leq 0 \Rightarrow \Delta G_{3} \leq 0, \\
\left|\mu^{\prime}\right|-\left|\mu_{1}\right|-|\bar{\mu}|=q_{3},\left|\beta^{\prime}\right|-\left|\beta_{2}\right|-|\bar{\beta}|=-q_{3} \Rightarrow \Delta G_{3} \leq-\frac{2}{3} q_{3},
\end{gathered}
$$

for a $q_{3} \geq 0$. This also applies to the interactions $\beta_{1}+\alpha_{2}+\beta_{2}, \beta_{1}+\mu_{2}+\beta_{2}$ and $\nu_{1}+\alpha_{2}+\nu_{2}$ which all have only one combination of outgoing waves and where the last two are increasing rarefaction-collisions.

We now turn to the interactions

$$
\begin{array}{ccc}
\alpha_{1}+\beta+\alpha_{2}+\nu, & \text { which is symmetric to } & \mu+\beta_{1}+\alpha+\beta_{2}, \\
\alpha_{1}+\nu+\alpha_{2}+\beta, & \text { which is symmetric to } & \alpha+\beta_{1}+\mu+\beta_{2} .
\end{array}
$$

These have two combination of outgoing waves, $\alpha^{\prime}+\nu^{\prime}$ and $\alpha^{\prime}+\beta^{\prime}$, and are divided into smaller steps;

$$
\begin{aligned}
\alpha_{1}+\left[\eta_{1}+\alpha_{2}\right]+\eta_{2} & \stackrel{\Delta G_{2}}{\longrightarrow} \alpha_{1}+\bar{\alpha}+\left[\bar{\eta}_{1}+\eta_{2}\right] \\
\stackrel{\Delta G_{3}}{\longrightarrow} & \alpha_{1}+\bar{\alpha}+\tilde{\alpha}+\tilde{\eta} \stackrel{\Delta G_{4}}{\longrightarrow} \alpha^{\prime}+\eta^{\prime},
\end{aligned}
$$


where $\eta_{1}$ and $\bar{\eta}_{1}$ are of the same type, whereas $\eta_{2}$ is not, and where $\tilde{\eta}$ and $\eta^{\prime}$ are of the same type. From Lemma 3.8 we have the needed estimates on $\Delta G_{2}$ and $\Delta G_{3}$. Due to property viii) of Lemma 2.1 we find for $q_{4}>0$ that

$$
\begin{array}{cl}
\left|\alpha^{\prime}\right|-|\tilde{\alpha}|-|\bar{\alpha}|-\left|\alpha_{1}\right|=-q_{4}, \quad\left|\nu^{\prime}\right|-|\tilde{\nu}| \leq q_{4} & \Rightarrow \quad \Delta G_{4} \leq-\frac{2}{3} q_{4}, \\
\left|\alpha^{\prime}\right|-|\tilde{\alpha}|-|\bar{\alpha}|-\left|\alpha_{1}\right| \leq 0, \quad\left|\beta^{\prime}\right|-|\tilde{\beta}| \leq 0 \quad \Rightarrow \quad \Delta G_{4} \leq 0 .
\end{array}
$$

This also covers the special cases $\beta_{1}+\alpha_{2}+\nu_{2}$ and $\nu_{1}+\alpha_{2}+\beta_{2}$. Note that if $\eta_{1}=\beta_{1}$, then $\left|\nu^{\prime}\right| \leq|\nu|$ by construction, so these interactions are actually not increasing rarefaction-collisions.

Then we are left with two interactions, each having four subcases,

$$
\mu+\beta+\alpha+\nu, \quad \text { and } \alpha+\mu+\nu+\beta .
$$

In the case with $\mu^{\prime}+\nu^{\prime}$ we divide the first interaction into smaller steps

$$
\alpha+[\nu+\mu]+\beta \stackrel{\Delta G_{2}}{\longrightarrow} \alpha+\bar{\mu}+\bar{\nu}+\beta \stackrel{\Delta G_{3}}{\longrightarrow} \mu^{\prime}+\nu^{\prime},
$$

where $\Delta G_{2} \leq 0$ by Lemma 3.8 and

$$
\left|\mu^{\prime}\right| \leq|\bar{\mu}| \leq|\mu|, \quad\left|\nu^{\prime}\right| \leq|\bar{\nu}| \leq|\nu| \quad \Rightarrow \quad \Delta G_{3} \leq 0
$$

follows from the properties of wave curves and estimate 3.15 . For the second interaction it follows by construction that

$$
\left|\mu^{\prime}\right|-|\mu| \leq 0, \quad\left|\nu^{\prime}\right|-|\nu| \leq 0, \quad \Rightarrow \quad \Delta G \leq 0 .
$$

In the case with $\alpha^{\prime}+\nu^{\prime}$, it follows by construction that the first interaction is an increasing rarefaction-collision where

$$
\left|\alpha^{\prime}\right|-|\alpha|=-q,\left|\nu^{\prime}\right|-|\nu| \leq q \Rightarrow \Delta G \leq-\frac{2}{3} q .
$$

For the second interaction we have $\left|\nu^{\prime}\right| \leq|\nu|$. We divide the interaction into smaller steps,

$$
\begin{gathered}
\mu+[\beta+\alpha]+\nu \stackrel{\Delta G_{2}}{\longrightarrow}[\mu+\bar{\alpha}]+\bar{\beta}+\nu \stackrel{\Delta G_{3}}{\longrightarrow} \tilde{\alpha}+[\tilde{\beta}+\bar{\beta}+\nu] \\
\stackrel{\Delta G_{4}}{\longrightarrow} \tilde{\alpha}+\hat{\alpha}+\hat{\nu} \stackrel{\Delta G_{5}}{\longrightarrow} \alpha^{\prime}+\nu^{\prime}
\end{gathered}
$$

where we have the necessary estimates on $\Delta G_{2}$ and $\Delta G_{3}$ from Lemma 3.8 and for $\Delta G_{4}$ by Lemma 3.9. By property viii) of Lemma 2.1 we find for a $q>0$ that

$$
\left|\alpha^{\prime}\right|-|\tilde{\alpha}|-|\hat{\alpha}|=-q,\left|\nu^{\prime}\right|-|\hat{\nu}|=q \Rightarrow \Delta G_{5} \leq 0 .
$$

The last two cases can be considered together. Note that for either interaction we obtain $\left|\nu^{\prime}\right| \leq|\nu|$ so that none of them are increasing rarefaction-collisions. We divide the interactions as follows;

$$
\begin{gathered}
\epsilon_{1}+\left[\eta_{1}+\epsilon_{2}\right]+\eta_{2} \stackrel{\Delta G_{2}}{\longrightarrow} \epsilon_{1}+\bar{\epsilon}+\left[\bar{\eta}+\eta_{2}\right] \stackrel{\Delta G_{3}}{\longrightarrow}\left[\epsilon_{1}+\bar{\epsilon}+\tilde{\alpha}\right]+\tilde{\beta} \\
\stackrel{\Delta G_{4}}{\longrightarrow} \hat{\epsilon}+\hat{\beta}+\tilde{\beta} \stackrel{\Delta G_{5}}{\longrightarrow} \epsilon^{\prime}+\beta^{\prime},
\end{gathered}
$$

where $\bar{\epsilon}$ is of the same type as $\epsilon_{2}, \bar{\eta}$ of same type as $\eta_{1}$, and $\hat{\epsilon}$ of the same type as $\epsilon^{\prime}$. From Lemma 3.8 and Lemma 3.9 we have estimates on $\Delta G_{2}, \Delta G_{3}$ and $\Delta G_{4}$, and by properties (viii) and (ix) of Lemma 2.1 we find

$$
\begin{gathered}
\left|\mu^{\prime}\right|-|\hat{\mu}|=q,\left|\beta^{\prime}\right|-|\hat{\beta}|-|\tilde{\beta}|=-q \Rightarrow \Delta G \leq 0, \\
\left|\alpha^{\prime}\right|-|\hat{\alpha}| \leq 0,\left|\beta^{\prime}\right|-|\hat{\beta}|-|\tilde{\beta}| \leq 0 \Rightarrow \Delta G \leq 0 .
\end{gathered}
$$


The interaction $\nu_{1}+\alpha_{2}+\beta_{2}$ has also four cases, and for all but one case, the above analysis apply. In the case $\alpha^{\prime}+\nu^{\prime}, \beta_{2}$ must cross $\nu$, and therefore there exist a $\hat{\nu}$ and a $\hat{\beta}$ so that $\hat{\nu}+\hat{\beta}$ connects $U_{l}$ to $U_{r}$ and

$$
|\hat{\nu}| \leq|\nu|, \quad|\hat{\beta}| \leq|\beta| \text {. }
$$

Then the interaction can be divided into the following steps,

$$
\nu_{1}+\mu_{2}+\beta_{2} \stackrel{\Delta G_{2}}{\longrightarrow}[\hat{\nu}+\hat{\beta}] \stackrel{\Delta G_{3}}{\longrightarrow} \alpha^{\prime}+\nu^{\prime},
$$

where $\Delta G_{2} \leq 0$ follows from 3.25 and $\Delta G_{3} \leq 0$ from Lemma 3.8. Note that $\left|\nu^{\prime}\right| \leq|\nu|$ by construction, so this is not an increasing rarefaction-collision.

This completes the discussion of all possible interactions at the second step, and thereby completes the proof.

Lemma 3.13. The functional $G$ decreases for all interactions of the form

$$
\sum_{i=1}^{n} \eta_{i}+\zeta+\sum_{i=1}^{m} \epsilon_{i} .
$$

Furthermore, $\Delta G \leq-\frac{2}{3} q$ for all increasing rarefaction-collisions where the strength of the outgoing rarefaction wave increases by $q>0$, and $\Delta G \leq-\frac{2}{3}\left|\theta^{\prime}\right|$ for new rarefaction-collisions where $\theta^{\prime}$ denotes the new outgoing rarefaction wave.

Proof. This is the general form for interactions possible in front tracking. All interactions without $\zeta$ are already covered by Lemma 3.12. Furthermore, Lemma 3.11 covers the interaction where $m=0$ (or $n=0$ ).

If $n>1$ and $m>1$, then we divide the interactions into smaller steps as follows

$$
\sum_{i=1}^{n} \eta_{i}+\zeta+\sum_{j=1}^{m} \epsilon_{j} \stackrel{\Delta G_{1}}{\longrightarrow}\left\{\begin{array}{l}
\mu_{1}+\beta_{1} \\
\alpha_{1}+\nu_{1} \\
\alpha_{1}+\beta_{1}
\end{array} \quad+\zeta+\left\{\begin{array}{l}
\mu_{2}+\beta_{2} \\
\alpha_{2}+\nu_{2} \\
\alpha_{2}+\beta_{2}
\end{array} \quad \stackrel{\Delta G_{n}}{\longrightarrow} \epsilon^{\prime}+\zeta+\eta^{\prime}\right.\right.
$$

where three of the nine possible combinations at the last step are symmetric to one of the other, thus, we have six different interactions to consider. If $n=1$ and $m>1$ (symmetric to $n>1$ and $m=1$ ), we get

$$
\eta_{1}+\zeta+\sum_{j=1}^{m} \epsilon_{j} \stackrel{\Delta G_{1}}{\longrightarrow} \eta_{1}+\zeta+\left\{\begin{array}{l}
\mu_{2}+\beta_{2} \\
\alpha_{2}+\nu_{2} \\
\alpha_{2}+\beta_{2}
\end{array} \quad \stackrel{\Delta G_{n}}{\longrightarrow} \epsilon^{\prime}+\zeta+\eta^{\prime},\right.
$$

with six different interactions at the second step. In addition, we have the four interactions where $m=n=1$,

$$
\eta_{1}+\zeta+\epsilon_{2} \stackrel{\Delta G_{n}}{\longrightarrow} \epsilon^{\prime}+\zeta+\eta^{\prime}
$$

where one is symmetric to one of the others.

An interaction that is symmetric to itself is referred to as a self-symmetric interaction. Three of the interactions given by (3.27) are self-symmetric. The other interactions have a symmetric interaction, and we choose to discuss the interactions starting with $\alpha_{1}+\nu_{1}$ over the ones starting with $\epsilon_{1}+\beta_{1}$, and the interaction starting with $\alpha_{1}+\beta_{1}$ over the one starting with $\mu_{1}+\beta_{1}$. None of the interactions given by 3.28 are symmetric to itself or to one of the other interactions. For the two interactions of the form $\epsilon+\zeta+\mu_{2}+\beta_{2}$, we will throughout this proof consider their symmetric interactions, $\alpha_{1}+\nu_{1}+\zeta+\eta_{2}$ instead. There are two self-symmetric interactions given by 3.29 . The remaining two interactions are symmetric, and we choose to discuss the one with $\eta_{1}=\nu_{1}$. 
We write the six interactions given by 3.27 of the general form

$$
\epsilon_{1}+\eta_{1}+\zeta+\epsilon_{2}+\eta_{2} \stackrel{\Delta G_{n}}{\longrightarrow} \epsilon^{\prime}+\zeta+\eta^{\prime} .
$$

If $\epsilon_{1}$ is of the same type as $\epsilon_{2}$ and $\eta_{1}$ is of the same type as $\eta_{2}$, then the interaction has only three possible combinations of outgoing fronts. Thus, two of the interactions given by 3.30 have three possible combination of outgoing fronts, while the remaining four interactions have four possible combination of outgoing waves

First, we consider the case where the outgoing fronts are $\mu^{\prime}+\zeta+\nu^{\prime}$. This is not a case for the interaction where all incoming fronts are shock-fronts. For the two interactions where $\epsilon_{1}+\eta_{1}$ is not equal to $\alpha_{1}+\nu_{1}$, one of the following estimates holds;

$$
\begin{aligned}
\left|\mu^{\prime}\right|-|\mu| \leq 0, \quad\left|\nu^{\prime}\right|-|\nu| \leq 0 & \Rightarrow \Delta G \leq 0, \\
\left|\mu^{\prime}\right|-|\mu| \leq|\beta|=q, \quad\left|\nu^{\prime}\right|-|\nu| \leq 0 & \Rightarrow \Delta G \leq-\frac{2}{3} q, \\
\left|\mu^{\prime}\right|-|\mu| \leq 0, \quad\left|\nu^{\prime}\right|-|\nu| \leq|\alpha|=q & \Rightarrow \Delta G \leq-\frac{2}{3} q .
\end{aligned}
$$

For the three interactions where $\epsilon_{1}+\eta_{1}=\alpha_{1}+\nu_{1}$, we need to divide the interactions into smaller steps;

$$
\begin{aligned}
+\epsilon_{2}+\eta_{2} & \stackrel{\Delta G_{2}}{\longrightarrow} \bar{\epsilon}+\zeta+\left[\bar{\nu}+\epsilon_{2}\right]+\eta_{2} \stackrel{\Delta G_{3}}{\longrightarrow} \bar{\epsilon}+[\zeta+\tilde{\epsilon}+\tilde{\nu}]+\eta_{2} \\
& \stackrel{\Delta G_{4}}{\longrightarrow} \bar{\epsilon}+\hat{\epsilon}+\zeta+\hat{\nu}+\eta_{2} \stackrel{\Delta G_{5}}{\longrightarrow} \mu^{\prime}+\zeta+\nu^{\prime}
\end{aligned}
$$

where $\tilde{\epsilon}$ and $\hat{\epsilon}$ are of the same type as $\epsilon_{2}$. If $\epsilon_{2}$ is a shock-front, then $\bar{\epsilon}$ is a rarefactionfront, otherwise, $\bar{\epsilon}$ can be of either type. From Lemma 3.8 and Lemma 3.9 we have estimates for $\Delta G_{i}$ for $i=2,3,4$. Furthermore, we have

$$
\left|\mu^{\prime}\right|-|\bar{\mu}|-|\hat{\mu}| \leq 0, \quad\left|\nu^{\prime}\right|-|\hat{\nu}| \leq 0 \Rightarrow \Delta G_{5} \leq 0,
$$

where $|\bar{\mu}|$ is only included if $\bar{\epsilon}$ is a rarefaction-front, and $|\hat{\nu}|$ only if $\hat{\epsilon}$ is a rarefactionfront.

Next, we consider interactions given by 3.28 and 3.29 . If all incoming fronts are shock-fronts, then $\mu^{\prime}+\zeta+\nu^{\prime}$ is not a possible combination of outgoing fronts. All interactions with $\eta_{1}=\nu_{1}$ can be divided into smaller steps similar to 3.32 , giving us the the same estimates for the last step. For the interactions with $\eta_{1}=\beta_{1}$, one of the estimates given by $(3.31)$ holds. Hence, the case $\mu^{\prime}+\zeta+\nu^{\prime}$ is covered for all interactions given by 3.28$)$ and $(3.29)$.

Let us now consider the case where the outgoing waves are $\mu^{\prime}+\zeta+\beta^{\prime}$. This combination is not possible for the interaction where the incoming 1-fronts are shock-fronts and the 3 -fronts are rarefaction-fronts. The other three interactions where the incoming 1 -fronts are shock-fronts, have at least one incoming 3 -shock and we have

$$
\left|\beta^{\prime}\right|-\sum_{\eta=\beta}\left|\eta_{i}\right| \leq-\left|\mu^{\prime}\right| \Rightarrow \Delta G \leq 0,
$$

where we sum over the strength of the incoming 3 -shocks. Thus, we are left with the interactions $\alpha+\nu+\zeta+\mu+\beta$ and $\mu+\beta+\zeta+\alpha+\nu$. First, note that if neither $\alpha$ nor $\mu^{\prime}$ intersects $\mu$ for the first interaction, or if $\nu$ or $\beta^{\prime}$ does intersect $\beta$ for the second, we have for a $q>0$ that

$$
\left|\mu^{\prime}\right|-|\mu| \leq q,\left|\beta^{\prime}\right|-|\beta|=-q \quad \Rightarrow \quad \Delta G \leq-\frac{2}{3} q .
$$

If $\alpha$ intersects $\mu$ for the first interaction, we can replace the interaction with a new one, still connecting the left state to the right state, as follows;

$$
\alpha+\nu+\zeta+\mu+\beta \stackrel{\Delta G_{2}}{\longrightarrow} \hat{\alpha}+[\zeta+\hat{\mu}+\beta] \stackrel{\Delta G_{3}}{\longrightarrow} \hat{\alpha}+\bar{\mu}+\zeta+\bar{\beta}
$$




$$
\stackrel{\Delta G_{4}}{\longrightarrow} \tilde{\mu}+\zeta+\tilde{\beta}+\bar{\beta} \stackrel{\Delta G_{5}}{\longrightarrow} \mu^{\prime}+\zeta+\beta^{\prime} .
$$

From Lemma 3.9 we have estimates for $\Delta G_{3}$ and $\Delta G_{4}$. Moreover, we have for a $q_{5}>0$ that

$$
\begin{aligned}
|\hat{\alpha}|-|\alpha| \leq 0,|\hat{\mu}|-|\mu| \leq 0 \quad & \Rightarrow \quad \Delta G_{2} \leq 0 \\
\left|\mu^{\prime}\right|-|\tilde{\mu}| \leq q_{5}, \quad\left|\beta^{\prime}\right|-|\tilde{\beta}|-|\bar{\beta}|=-q_{5} & \Rightarrow \quad \Delta G_{5} \leq-\frac{2}{3} q_{5} .
\end{aligned}
$$

If $\mu^{\prime}$ intersects $\mu$, we use a similar approach and replace the interaction with a new one,

$$
\begin{aligned}
\alpha+\nu+\zeta+\mu+\beta & \stackrel{\Delta G_{2}}{\longrightarrow} \hat{\mu}_{1}+\left[\zeta+\hat{\mu}_{2}+\beta\right] \\
& \stackrel{\Delta G_{3}}{\longrightarrow} \hat{\mu}_{1}+\bar{\mu}+\zeta+\bar{\beta} \stackrel{\Delta G_{4}}{\longrightarrow} \mu^{\prime}+\zeta+\beta^{\prime},
\end{aligned}
$$

where we have estimate for $\Delta G_{3}$ due to Lemma 3.9. Moreover,

$$
\begin{aligned}
\left|\hat{\mu}_{1}\right|+\left|\hat{\mu}_{2}\right|-|\mu| \leq 0 & \Rightarrow \quad \Delta G_{2} \leq 0, \\
\left|\mu^{\prime}\right|-|\bar{\mu}|-\left|\hat{\mu}_{1}\right|=0,\left|\beta^{\prime}\right|-|\bar{\beta}|=0 & \Rightarrow \quad \Delta G_{5} \leq 0 .
\end{aligned}
$$

If no fronts intersect for the second interaction, we cannot apply a clever replacement. Thus, we divide the interaction into several smaller steps,

$$
\begin{aligned}
{[\mu+\beta+\zeta]+\alpha+\nu } & \stackrel{\Delta G_{2}}{\longrightarrow} \bar{\mu}+\zeta+[\bar{\beta}+\alpha]+\nu \stackrel{\Delta G_{3}}{\longrightarrow} \bar{\mu}+[\zeta+\tilde{\alpha}+\tilde{\beta}]+\nu \\
& \stackrel{\Delta G_{4}}{\longrightarrow} \bar{\mu}+\hat{\alpha}+[\zeta+\hat{\beta}+\nu] \stackrel{\Delta G_{5}}{\longrightarrow}[\bar{\mu}+\hat{\alpha}+\overline{\bar{\alpha}}+\zeta]+\overline{\bar{\beta}} \\
& \stackrel{\Delta G_{6}}{\longrightarrow} \check{\mu}+\zeta+\check{\beta}+\overline{\bar{\beta}} \stackrel{\Delta G_{7}}{\longrightarrow} \mu^{\prime}+\zeta+\beta^{\prime},
\end{aligned}
$$

where we have estimates for $\Delta G_{i}, i=2, \ldots, 6$, from Lemma 3.8 and Lemma 3.9 Furthermore, we have for a $q_{7}>0$ that

$$
\left|\mu^{\prime}\right|-|\check{\mu}| \leq q_{7},\left|\beta^{\prime}\right|-|\check{\beta}|-|\overline{\bar{\beta}}|=q_{7} \quad \Rightarrow \quad \Delta G_{7} \leq-\frac{2}{3} q_{7} .
$$

Similarly, $\mu^{\prime}+\zeta^{\prime}+\beta^{\prime}$ is not a case for interactions given by $(3.28$ and 3.29 where the incoming 1 -fronts are shock-fronts and the incoming 3 -fronts are rarefactionfronts. Moreover, all interactions where $\epsilon_{2}=\alpha_{2}$ have estimate given by (3.33). The remaining two interactions are special cases of $\alpha_{1}+\nu_{1}+\zeta+\mu_{2}+\beta_{2}$ where either $\alpha_{1}$ or $\beta_{2}$ is missing, and they are covered by the above discussion.

Observe that for the self-symmetric interactions the case where the outgoing waves are $\mu^{\prime}+\zeta+\beta^{\prime}$ is symmetric to the case where the outgoing waves are $\alpha^{\prime}+\zeta+\nu^{\prime}$. Thus, only one case is left for these three interactions, the case where we have $\alpha^{\prime}+\zeta+\beta^{\prime}$. Some configurations of fronts gives us

$$
\left|\alpha^{\prime}\right|-|\alpha| \leq 0,\left|\beta^{\prime}\right|-|\beta| \leq 0 \quad \Rightarrow \quad \Delta G \leq 0,
$$

directly. Otherwise, we have to divide the interactions into smaller steps as follows

$$
\begin{aligned}
\epsilon_{1}+\eta_{1}+\left[\zeta+\epsilon_{2}+\eta_{2}\right] & \stackrel{\Delta G_{2}}{\longrightarrow} \epsilon_{1}+\left[\eta_{1}+\bar{\epsilon}\right]+\zeta+\bar{\eta} \stackrel{\Delta G_{3}}{\longrightarrow} \epsilon_{1}+[\tilde{\epsilon}+\tilde{\eta}+\zeta]+\bar{\eta} \\
& \stackrel{\Delta G_{4}}{\longrightarrow}\left[\epsilon_{1}+\hat{\epsilon}+\zeta\right]+\hat{\eta}+\bar{\eta} \stackrel{\Delta G_{5}}{\longrightarrow} \overline{\bar{\alpha}}+[\zeta+\overline{\bar{\eta}}+\hat{\eta}+\bar{\eta}] \\
& \stackrel{\Delta G_{6}}{\longrightarrow} \overline{\bar{\alpha}}+\check{\epsilon}+\zeta+\check{\beta} \stackrel{\Delta G_{7}}{\longrightarrow} \alpha^{\prime}+\zeta+\beta^{\prime},
\end{aligned}
$$

where $\bar{\epsilon}$ and $\tilde{\epsilon}$ are of the same type as $\epsilon_{2}$, and $\tilde{\eta}$ is of the same type as $\eta_{1}$. If $\eta_{1}$ is a rarefaction-front, then $\bar{\eta}$ is a shock-front, $\hat{\epsilon}$ can be of either type, and $\overline{\bar{\eta}}$ will be of the opposite type of $\hat{\epsilon}$. If $\eta_{1}$ is a shock-front, then $\bar{\eta}$ can be of either type, $\hat{\epsilon}=\hat{\alpha}$, and $\overline{\bar{\eta}}$ is of the opposite type as $\epsilon_{1}$. Moreover, if $\overline{\bar{\eta}}$ and $\hat{\eta}$ are both rarefaction-fronts, we add them together to one 3-rarefaction wave according to property (i) of Lemma 2.1. If all 3 -fronts at step six are shock-fronts, then $\check{\epsilon}=\check{\mu}$ and does not take part in the 
estimate below, otherwise $\check{\epsilon}=\check{\alpha}$. We have estimates for $\Delta G_{i}, i=2, \ldots, 6$, due to Lemma 3.8 and 3.9 . Moreover,

$$
\left|\alpha^{\prime}\right|-|\overline{\bar{\alpha}}|-|\check{\alpha}| \leq 0,\left|\beta^{\prime}\right|-|\check{\beta}| \leq 0 \quad \Rightarrow \quad \Delta G_{7} \leq 0 .
$$

None of the interactions given by (3.28) are self-symmetric. For interactions given by $(3.29)$, one of the self-symmetric interactions involve only rarefaction-fronts and $\alpha^{\prime}+\zeta+\beta^{\prime}$ is therefore not a possible case. The other self-symmetric interaction, $\beta+\zeta+\alpha$, can be divided into smaller steps as above and is thus covered by the estimates just given. Here, step four is redundant.

Finally, we consider the last two cases, when the outgoing waves are $\alpha^{\prime}+\zeta+\nu^{\prime}$ or $\alpha^{\prime}+\zeta+\beta^{\prime}$, for the three interactions that are not self-symmetric. These interactions all have two incoming shock-fronts of the first family, and we observe that

$$
\text { if }\left|\alpha^{\prime}\right|-\left|\alpha_{1}\right|-\left|\alpha_{2}\right|=-q \leq 0 \text {, then } \begin{cases}\left|\nu^{\prime}\right|-|\nu| \leq q & \Rightarrow \Delta G \leq-\frac{2}{3} q, \\ \left|\beta^{\prime}\right|-|\beta| \leq 0 & \Rightarrow \Delta G \leq 0 .\end{cases}
$$

If this condition of the 1-shocks does not hold, we divide the interactions into smaller steps as follows;

$$
\begin{aligned}
\alpha_{1}+\eta_{1}+\left[\zeta+\alpha_{2}+\eta_{2}\right] & \stackrel{\Delta G_{2}}{\longrightarrow} \alpha_{1}+\left[\eta_{1}+\bar{\alpha}\right]+\zeta+\bar{\eta} \stackrel{\Delta G_{3}}{\longrightarrow} \alpha_{1}+[\tilde{\alpha}+\tilde{\eta}+\zeta]+\bar{\eta} \\
& \stackrel{\Delta G_{4}}{\longrightarrow}\left[\alpha_{1}+\hat{\epsilon}+\zeta\right]+\hat{\eta}+\bar{\eta} \stackrel{\Delta G_{5}}{\longrightarrow} \overline{\bar{\alpha}}+[\zeta+\overline{\bar{\eta}}+\hat{\eta}+\bar{\eta}] \\
& \stackrel{\Delta G_{6}}{\longrightarrow} \overline{\bar{\alpha}}+\check{\alpha}+\zeta+\check{\eta} \stackrel{\Delta G_{7}}{\longrightarrow} \alpha^{\prime}+\zeta+\eta^{\prime},
\end{aligned}
$$

where $\tilde{\eta}$ and $\hat{\eta}$ are of the same type as $\eta_{1}$, and $\check{\eta}$ is of the same type as $\eta^{\prime}$. Furthermore, if $\eta_{1}$ and $\eta_{2}$ are rarefaction-fronts and $\eta^{\prime}$ is a shock-front, then $\bar{\eta}$ is also a shock-front. If $\eta_{2}$ and $\eta^{\prime}$ are of the same type, while $\eta_{1}$ is not, then $\bar{\eta}$ is of the same type as $\eta_{2}$. Otherwise, $\bar{\eta}$ can be of either type. Likewise, $\hat{\epsilon}$ can be of either type, and we therefore have to include step five, where $\overline{\bar{\eta}}$ is not of the same type as $\hat{\epsilon}$. Moreover, if at some point, two adjacent 3-fronts are both rarefaction-fronts, then we add them together to one 3 -rarefaction wave according to property (i) of Lemma 2.1. Finally, if all 3 -fronts at step six, that is, $\overline{\bar{\eta}}, \hat{\eta}$ and $\bar{\eta}$, are all of the same type as $\eta^{\prime}$, then we skip step six and replace the strength of $\check{\eta}$ in the estimates below by the sum of the strength of these 3-fronts. From Lemma 3.8 and 3.9 we have estimates for $\Delta G_{i}, i=2, \ldots, 6$. Finally, we have

$$
\begin{array}{cll}
\left|\alpha^{\prime}\right|-|\overline{\bar{\alpha}}|-|\check{\alpha}|=q_{7},\left|\nu^{\prime}\right|-|\check{\nu}| \leq q_{7} & \Rightarrow & \Delta G_{7} \leq-\frac{2}{3} q_{7}, \\
\left|\alpha^{\prime}\right|-|\overline{\bar{\alpha}}|-|\check{\alpha}| \leq 0,\left|\beta^{\prime}\right|-|\check{\beta}| \leq 0 & \Rightarrow & \Delta G_{7} \leq 0,
\end{array}
$$

for the two cases, respectively.

The interactions given by $(3.28)$ and $(3.29)$ can be divided into smaller steps in the same way. For interactions with no $\epsilon_{1}$, we in general only need six steps because step four is not needed. However, if $\hat{\epsilon}=\hat{\mu}$, we may have to interchange step four and five, so that we still have seven steps. Moreover, if

$$
\left|\alpha^{\prime}\right|-|\alpha| \leq-\left|\nu^{\prime}\right|, \text { then } \Delta G \leq 0,
$$

without dividing the interaction into smaller steps.

All cases for all interactions are now considered, thus, we have proved the lemma.

This concludes the discussion of all interactions between physical fronts. For interactions generating non-physical fronts and interactions where one of the incoming fronts is non-physical, we have: 
Lemma 3.14. For an interaction where a non-physical front is generated, we have

$$
\Delta G \leq-3 k\left|\theta^{n p}\right|, \text { and }\left|\theta^{n p}\right| \leq \frac{4}{3} c_{2} \rho
$$

where the positive constants $k$ and $c_{2}$ depend only on $p_{\min }, p_{\max }$, and $\bar{\gamma}$. Moreover, $\Delta G \leq 0$ for all interactions with incoming non-physical fronts, and the strength of a non-physical front does not change in interactions.

Proof. The simplified Riemann solver is used for interactions of the type $\zeta+$ $\sum_{i=1}^{n} \epsilon_{i}$, and the symmetric interactions, where condition 3.1 holds, that is, where $|\zeta| \sum_{i}\left|\epsilon_{i}\right| \leq \rho$. Since non-physical fronts cannot be generated in any other interactions, it follows from Lemma 3.8 and Lemma 3.11 that $\Delta G \leq-3 k\left|\theta^{\text {np }}\right|$. By estimates 3.17), 3.18, and 3.23) established in the proofs of these lemmas, we have

$$
\left|\theta^{\mathrm{np}}\right| \leq \frac{4}{3} c_{2}|\zeta| \sum_{i}\left|\epsilon_{i}\right| \leq \frac{4}{3} c_{2} \rho .
$$

Whenever a non-physical front is involved in an interaction, we let the nonphysical front pass through the interaction without changing its strength. Then we solve the remaining interaction. The non-physical front only introduces a shift in the $u$-variable, and since all wave curves are invariant under a transformation in $u$, the interaction and all its estimates are the same as if it was not shifted. Hence, by Lemma 3.8 through Lemma 3.13 , and the fact that the non-physical front plays no role in $G$, we have $\Delta G \leq 0$ for all interactions with an incoming non-physical front. This also applies to interactions having two incoming non-physical fronts, one with negative and one with positive speed. In particular we have $\Delta G=0$ when a non-physical front collides with one other front, physical or non-physical; they just pass through each other, continuing with the same strength.

We have now established that $\Delta G \leq 0$ for all possible interactions and can finally prove that $G$ is decreasing in time.

Proof of Proposition 3.7. By Lemma 3.8 through Lemma 3.14 it follows that $G$ decreases for all possible interactions, and, in particular, that $\Delta G \leq-\frac{2}{3} q$ for all increasing rarefaction-collisions where the strength of the rarefaction wave increases by $q, \Delta G \leq-\frac{2}{3}\left|\theta^{\prime}\right|$ for all new rarefaction-collisions where $\theta^{\prime}$ denotes the new rarefaction wave, and $\Delta G \leq-3 k\left|\theta^{\text {np }}\right|$ for all interactions generating a non-physical front. Finally, since $G$ decreases, it follows from 3.13 that $F\left(t_{n}\right) \leq \frac{5}{3} L\left(t_{0}\right)$.

3.3. Finite number of interactions. The next step is to show that the fronttracking algorithm generates an approximate solution in a finite number of steps. We do this by proving that there is a finite number of physical and non-physical fronts, and, hence, a finite number of interactions.

As discussed in Subsection 3.1, the number of fronts increases when we have a $\gamma$-collision solved by the approximate solver, or when a rarefaction wave splits. Moreover, splitting of rarefaction waves can only be caused by new rarefactioncollisions or increasing rarefaction-collisions, and we now show that the number of such interactions is finite.

Lemma 3.15. For a fixed $\delta$, there is only a finite number of new rarefactioncollisions where the new rarefaction wave splits into two or more fronts.

Proof. From Proposition 3.7 we have $\Delta G \leq-\frac{2}{3}|\theta|$ for all new rarefaction-collisions, where $\theta$ is the new outgoing rarefaction wave. This was proved in Subsection 3.2 where all interactions of this type were identified. The new rarefaction wave splits into two or more fronts only if its strength, $|\theta|$, is larger than $\delta$. Hence, $\Delta G \leq-\frac{2}{3} \delta$ across a new rarefaction-collision where the new rarefaction wave splits. Since 
$G$ is a decreasing, non-negative functional, there can only be a finite number of interactions where $G$ decreases by at least $\frac{2}{3} \delta$. This proves the lemma.

We next consider the increasing rarefaction-collisions and look at the change in $G$ from a split rarefaction-front appears until it has gained enough strength to split again. That is, consider an increasing rarefaction-collision at $t=\tau_{1}$ where the outgoing rarefaction wave splits into several fronts. Let $\tau_{n}$ be the collision time when the first of the split rarefaction-fronts splits again after gaining strength through increasing rarefaction-collisions. Fix two time lines, $t_{i}$ and $t_{j}$, so that $t_{i}<\tau_{1}<\tau_{n}<t_{j}$. Assume that the only rarefaction-front crossing $t=t_{i}$ that results in a split rarefaction wave (through increasing rarefaction-collisions) before $t=t_{j}$, is the rarefaction-front colliding at $t=\tau_{1}$. Define $\Delta G_{\text {split }}:=G\left(t_{j}\right)-G\left(t_{i}\right)$.

Lemma 3.16. Let $t_{i}<\tau_{1}<\tau_{n}<t_{j}$ and $\Delta G_{\text {split }}$ be as defined above. Then, for a fixed $\delta$,

$$
\Delta G_{\text {split }} \leq-\frac{1}{3} \delta
$$

Furthermore, there is only a finite number of increasing rarefaction-collisions where the increasing rarefaction wave splits into two or more fronts.

Proof. From Proposition 3.7 we have $\Delta G \leq-\frac{2}{3} q$ for all increasing rarefactioncollisions where $q>0$ bounds the increase of the strength of the rarefaction wave, that is, the strength of the outgoing rarefaction wave is less than or equal to $q$ plus (the sum of) the strength(s) of the incoming rarefaction-front(s) of the same family.

Let $\theta^{\prime}$ be the outgoing rarefaction wave of the increasing rarefaction collision at $t=\tau_{1}$, thus, $\theta^{\prime}$ splits, and let furthermore $\theta_{0}$ be the incoming rarefaction-front of the same family. By the assumptions, $\left|\theta_{0}\right|=a \delta$ for $0<a \leq 1$, and

$$
\delta<\left|\theta^{\prime}\right| \leq\left|\theta_{0}\right|+q_{1} .
$$

Furthermore, $\Delta G_{1} \leq-\frac{2}{3} q_{1}$ across this interaction. Let $m$ be the number of fronts $\theta^{\prime}$ splits into and let furthermore $\theta_{1}$ denote the first of these split fronts that gain enough strength to split again. Thus,

$$
\left|\theta_{1}\right|=\frac{1}{m}\left|\theta^{\prime}\right| \leq \frac{\left|\theta_{0}\right|+q_{1}}{m}=\frac{a \delta+q_{1}}{m} .
$$

We follow this rarefaction-front until it splits again after an interaction at $t=\tau_{n}$,

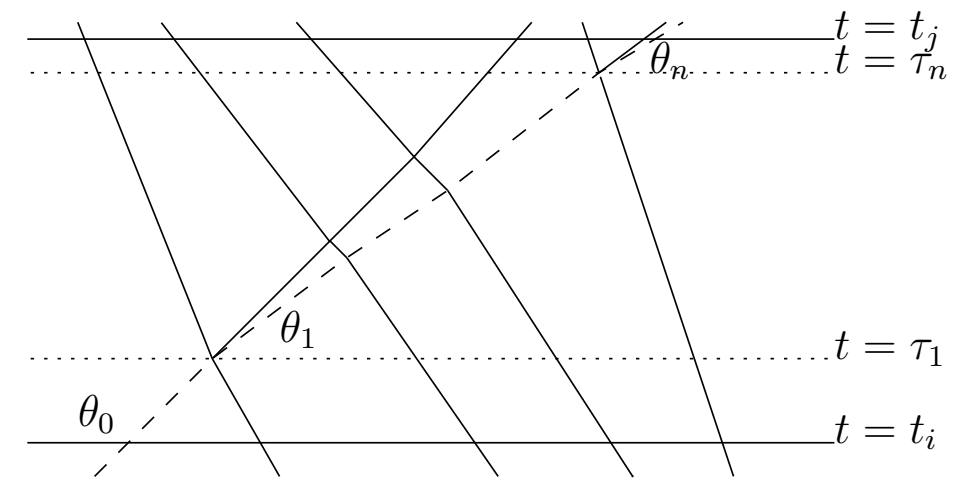

FIGURE 17. An illustration of several increasing rarefactioncollision where a rarefaction wave splits at $t=\tau_{1}$ and $t=\tau_{n}$.

and in Figure 17, where $m=2$, this front is drawn by dashed lines. The only way for 
the rarefaction-front to gain strength is through increasing rarefaction-collisions, all other interactions only weaken the rarefaction-front. We can therefore assume that the rarefaction-front we follow is only involved in increasing rarefaction-collisions up to $t=t_{j}$. For each interaction the strength of the rarefaction-front increases by at most $q_{i}$, thus,

$$
\left|\theta_{n}\right| \leq\left|\theta_{n-1}\right|+q_{n} \leq\left|\theta_{1}\right|+\sum_{2}^{n} q_{k} \leq \frac{a \delta+q_{1}}{m}+\sum_{2}^{n} q_{k} \leq \frac{a}{m} \delta+\sum_{1}^{n} q_{k},
$$

and $\Delta G_{k} \leq-\frac{2}{3} q_{k}$ for $k=1, \ldots, n$. By definition, $\tau_{n}$ is the first collision time after $\tau_{1}$ where a rarefaction wave splits, thus,

$$
\left|\theta_{n-1}\right| \leq \delta<\left|\theta_{n}\right|
$$

All interactions taking place between $t_{i}$ and $t_{j}$ have $\Delta G \leq 0$ by Proposition 3.7 . Combining (3.36) and (3.37) we get

$$
\begin{aligned}
\Delta G_{\text {split }} & =\sum_{\tau_{k}} \Delta G\left(\tau_{k}\right)+\sum_{\tau \neq \tau_{k}} \Delta G(\tau) \leq \sum_{\tau_{k}} \Delta G\left(\tau_{k}\right) \\
& \leq \sum_{i=1}^{n}-\frac{2}{3} q_{k} \leq-\frac{2}{3}\left(1-\frac{a}{m}\right) \delta \leq-\frac{1}{3} \delta
\end{aligned}
$$

where we have used that $0<a \leq 1$ and $m \geq 2$. This proves (3.35).

Furthermore, $G$ is non-negative and decreases by at least $\frac{1}{3} \delta$ from the time when a rarefaction wave splits due to an increasing rarefaction-collision until the time when the first of the split fronts has gained enough strength to split again. Hence, this can only happen a finite number of times, and therefore, there can only be a finite number of increasing rarefaction-collisions where the rarefaction wave splits.

Thus, by Lemma 3.15 and Lemma 3.16, there is a finite number of interactions resulting in split rarefaction waves. That is, there is only a finite number of interactions with more than one outgoing front of each family.

The only other interactions with more outgoing physical fronts than incoming fronts, are $\gamma$-collisions solved by the approximate solver. These interaction are solved by the approximate solver if $|\theta||\zeta|>\rho$, where $\theta$ is the incoming 1- or 3front. By Proposition 3.7, $G$ decreases and, and in particular, $\Delta G \leq 0$ for all interactions. Therefore,

$$
0<G\left(t_{n}\right) \leq G\left(t_{0}\right)+\sum_{\tau<t_{n}} \Delta G(\tau) \leq G\left(t_{0}\right)+\sum_{\tau_{\gamma}<t_{n}} \Delta G\left(\tau_{\gamma}\right),
$$

where $\tau$ is any collision time and $\tau_{\gamma}$ is the collision time of a $\gamma$-collision solved by the approximate solver. Furthermore, Lemma 3.8 states that $\Delta G \leq-\frac{1}{9}|\theta||\zeta|$ for all $\gamma$-collisions, thus,

$$
\sum_{\tau_{\gamma}<t_{n}}|\theta||\zeta| \leq-9 \sum_{\tau_{\gamma}<t_{n}} \Delta G\left(\tau_{\gamma}\right) \leq 9 G\left(t_{0}\right) .
$$

This estimate is true for all $t_{n}<\infty$, hence, there is at most $9 G\left(t_{0}\right) / \rho$ number of $\gamma$ collisions where $|\theta||\zeta|>\rho$, that is, where a $\gamma$-collision is solved by the approximate solver. These are the only interactions creating more physical fronts in addition to the finite number of split rarefaction waves. Thus, the number of physical fronts remains finite for all times. Moreover, non-physical fronts are only generated when physical fronts interact with a contact discontinuity. Each physical front can only interact once with a given contact discontinuity, and there is a finite number of contact discontinuities, hence, there is a finite number of interactions generating non-physical fronts. 
In other words, there is a finite number of physical and non-physical fronts for any given time, and these fronts can only interact a finite number of times. Thus, front tracking gives us an approximate solution in a finite number of steps for any $t \in(0, \infty)$.

3.4. The total amount of non-physical fronts. In order to prove that the sequence of approximate solutions converges to a weak solution, we need to estimate the total amount of non-physical fronts introduced. First of all, we assign a generation to all fronts except the contact discontinuities.

All initial 1- and 3-fronts are of generation one. The outgoing front of an interaction has the lowest generation of the incoming fronts of the same family. If there is no incoming front of the same family, the outgoing front has a generation one higher than the highest generation of the incoming fronts. If there is a non-physical front generated in an interaction, its generation is also one higher than the highest generation of the incoming fronts. If an interaction has an incoming non-physical front, the outgoing non-physical front has the same generation as the incoming, thus, the generation of a non-physical front never changes once it is created. Furthermore, all fronts part of a split rarefaction wave have the same generation as the rarefaction wave.

From the results in Proposition 3.7 we have

$$
0<G\left(t_{n}\right)=G\left(t_{0}\right)+\sum_{\tau \leq t_{n}} \Delta G(\tau) \leq G\left(t_{0}\right)+\sum_{\tau_{\mathrm{np}} \leq t_{n}} \Delta G\left(\tau_{\mathrm{np}}\right),
$$

where $\tau_{\mathrm{np}}$ is a collision time when a non-physical front is generated. Moreover, by Lemma 3.14 we have $\Delta G \leq-3 k\left|\theta^{\text {np }}\right|$ for all interactions where a non-physical front is generated. Let $\theta_{i}^{\mathrm{np}}$ denote a non-physical front of generation $i$. Since neither the strength of a non-physical front, nor its generation, changes due to interactions, we get

$$
\sum_{i} \sum_{t=t_{n}}\left|\theta_{i}^{\mathrm{np}}\right|=\sum_{\tau_{\mathrm{np}} \leq t_{n}}\left|\theta^{\mathrm{np}}\right| \leq-\frac{1}{3 k} \sum_{\tau_{\mathrm{np}} \leq t_{n}} \Delta G\left(\tau_{\mathrm{np}}\right) \leq \frac{G\left(t_{0}\right)}{3 k} .
$$

Thus, there exists a $j$ so that

$$
\sum_{i>j} \sum_{t=t_{n}}\left|\theta_{i}^{\mathrm{np}}\right|=\mathcal{O}(1) \delta_{\text {init }}
$$

Let $N_{j}$ be the number of fronts of generation less than or equal to $j$. From the previous section we know that $N_{j}$ is finite. and according to [4, Ch. 7.3],

$$
N_{j} \leq P_{j}\left(N_{0}, \delta^{-1}\right),
$$

where $P_{j}$ is a polynomial function of $\delta^{-1}$ and the number of initial fronts, $N_{0}$. Using this, we can now prove the following:

Lemma 3.17. For any given $\delta_{\text {init }}>0$ there exists a $\rho>0$ so that

$$
\sum_{t=t_{n}}\left|\theta^{n p}\right|=\mathcal{O}\left(\delta_{\text {init }}\right) \text {. }
$$

Proof. Fix a $j=j_{0}$ so that 3.39 holds. The number of non-physical fronts of generation less than or equal to $j_{0}$ is less than $N_{j_{0}}$, which again is bounded by $P_{j_{0}}\left(N_{0}, \delta^{-1}\right)$. We therefore get

$$
\begin{aligned}
\sum_{t=t_{n}}\left|\theta^{\mathrm{np}}\right| & \leq \sum_{i \leq j_{0}} \sum_{t=t_{n}}\left|\theta_{i}^{\mathrm{np}}\right|+\sum_{i>j_{0}} \sum_{t=t_{n}}\left|\theta_{i}^{\mathrm{np}}\right| \\
& \leq \frac{4}{3} c_{2} \rho P_{j_{0}}\left(N_{0}, \delta^{-1}\right)+\mathcal{O}(1) \delta_{\text {init }}=\mathcal{O}\left(\delta_{\text {init }}\right)
\end{aligned}
$$

by choosing $\rho$ so that $\frac{4}{3} c_{2} \rho P_{j_{0}}\left(N_{0}, \delta^{-1}\right)=\mathcal{O}\left(\delta_{\text {init }}\right) t$. 
Recall that $\delta_{\text {init }}=\mathcal{O}(\delta)$, so that $\delta_{\text {init }} \rightarrow 0$ when $\delta \rightarrow 0$.

3.5. Bounded total variation. We have established that if condition $(3.12)$ is satisfied, then $G$ is decreasing and $U^{\delta}$ can be defined up to any time. The next step is to bound the total variation of $U^{\delta}$.

From Section 3.2 we recall that $C_{1}$ is the constant appearing in estimate 3.16 , $C_{2}$ is given by (3.4), $k$ by (3.5), and $C$ by (3.11). Define the constant

$$
\kappa:=1+\frac{10}{9}\left(3 s_{\max }^{\prime} k+1\right)
$$

where $s_{\max }^{\prime}$ is the upper bound of $\partial s / \partial p$, cf. property $\mathrm{v}$ of Lemma 2.1. Given these constants, that only depend on $p_{\min }, p_{\max }$ and $\bar{\gamma}$, we can state the following result.

Lemma 3.18. If the initial data satisfy

$$
(\bar{\gamma}-1) \mathrm{T} . \mathrm{V} \cdot\left(p_{0}, u_{0}\right) \leq \frac{C}{9 k C_{1}} \text { and T.V. }\left(\gamma_{0}\right) \leq \frac{C}{9 C_{2}}
$$

and the approximate solution $U^{\delta}(x, t)=\left(p^{\delta}(x, t), u^{\delta}(x, t), \gamma^{\delta}(x, t)\right)$ obtained using front tracking is bounded away from vacuum, then

$$
\begin{gathered}
\mathrm{T} . \mathrm{V} \cdot\left(p^{\delta}(\cdot, t), u^{\delta}(\cdot, t)\right) \leq 2 \kappa k \mathrm{~T} . \mathrm{V} \cdot\left(p_{0}, u_{0}\right), \\
\mathrm{T} . \mathrm{V} \cdot\left(\gamma^{\delta}(\cdot, t)\right) \leq \mathrm{T} . \mathrm{V} \cdot\left(\gamma_{0}\right) .
\end{gathered}
$$

Proof. First, 3.42 is obvious since $\gamma$ only changes along contact discontinuities, thus,

$$
\mathrm{T} . \mathrm{V} \cdot\left(\gamma^{\delta}\left(\cdot, t_{n}\right)\right)=F_{\gamma}=\mathrm{T} . \mathrm{V} \cdot\left(\gamma^{\delta}(\cdot, 0)\right) \leq \mathrm{T} \cdot \mathrm{V} \cdot\left(\gamma_{0}\right),
$$

for any time line $t=t_{n}$. Furthermore,

$$
L\left(t_{0}\right) \leq \mathrm{T} . \mathrm{V} \cdot\left(p^{\delta}(\cdot, 0)\right)+k \mathrm{~T} . \mathrm{V} \cdot\left(u^{\delta}(\cdot, 0)\right) \leq k \mathrm{~T} . \mathrm{V} \cdot\left(p^{\delta}(\cdot, 0), u^{\delta}(\cdot, 0)\right),
$$

for $t_{0}=0+$. Whenever 3.40 is satisfied, we therefore have

$$
\begin{gathered}
L\left(t_{0}\right) \leq k \mathrm{~T} \cdot \mathrm{V} \cdot\left(p^{\delta}(\cdot, 0), u^{\delta}(\cdot, 0)\right) \leq k \mathrm{~T} \cdot \mathrm{V} \cdot\left(p_{0}, u_{0}\right) \leq \frac{C}{9 C_{1}(\bar{\gamma}-1)}, \\
F_{\gamma}=\mathrm{T} . \mathrm{V} \cdot\left(\gamma^{\delta}(\cdot, 0)\right) \leq \mathrm{T} \cdot \mathrm{V} \cdot\left(\gamma_{0}\right) \leq \frac{C}{9 C_{2}},
\end{gathered}
$$

thus, by Proposition 3.7 the Glimm functional is decreasing and $F\left(t_{n}\right) \leq \frac{5}{3} L\left(t_{0}\right)$. We use this to bound T.V. $\left(u^{\delta}\left(\cdot, t_{n}\right)\right)$. If there were no non-physical fronts, we would have

$$
\sum_{\mathrm{rf}} \llbracket u \rrbracket=\sum_{\text {shock }} \llbracket u \rrbracket+u(\infty, \cdot)-u(-\infty, \cdot),
$$

because $u$ is increasing along all rarefaction waves and decreasing along all shock waves. Here $\llbracket u \rrbracket:=\left|u_{r}-u_{l}\right|$ for a wave connecting $U_{l}$ to $U_{r}$, and $\mathrm{rf}$ is short for rarefaction wave. Let $u_{ \pm}=u_{0}( \pm \infty)$ and define

$$
c_{0}:=|u(\infty, \cdot)-u(-\infty, \cdot)|=\left|u_{+}-u_{-}\right|,
$$

since $u( \pm \infty, \cdot)=u_{0}( \pm \infty)$. Including the non-physical fronts, we have

$$
\sum_{\mathrm{rf}} \llbracket u \rrbracket \leq \sum_{\text {shock }} \llbracket u \rrbracket+\sum_{\mathrm{np}} \llbracket u \rrbracket+c_{0},
$$

where "np" is short for non-physical front. Thus,

$$
\begin{aligned}
\mathrm{T} . \mathrm{V} \cdot\left(u^{\delta}\left(\cdot, t_{n}\right)\right) & =\sum_{\mathrm{rf}} \llbracket u \rrbracket+\sum_{\text {shock }} \llbracket u \rrbracket+\sum_{\mathrm{np}} \llbracket u \rrbracket \leq 2 \sum_{\text {shock }} \llbracket u \rrbracket+2 \sum_{\mathrm{np}} \llbracket u \rrbracket+c_{0} \\
& \leq 2 \sum_{\text {shock }}\left|s^{\prime}\left(\tilde{p}, p_{l}, \gamma_{l}\right)\right| \llbracket p \rrbracket+\frac{2}{3 k} G\left(t_{0}\right)+c_{0}
\end{aligned}
$$




$$
\begin{aligned}
& \leq 2 s_{\text {max }}^{\prime} \sum_{\text {shock }} \llbracket p \rrbracket+\frac{10}{9 k} L\left(t_{0}\right)+c_{0}=2 s_{\max }^{\prime} F\left(t_{n}\right)+\frac{10}{9 k} L\left(t_{0}\right)+c_{0} \\
& \leq 2 s_{\max }^{\prime} \frac{5}{3} L\left(t_{0}\right)+\frac{10}{9 k} L\left(t_{0}\right)+c_{0} \leq \frac{10}{9}\left(3 s_{\max }^{\prime} k+1\right) \mathrm{T} \cdot \mathrm{V} \cdot\left(p_{0}, u_{0}\right)+c_{0} \\
& \leq \kappa \mathrm{T} \cdot \mathrm{V} \cdot\left(p_{0}, u_{0}\right),
\end{aligned}
$$

where we have used that $c_{0} \leq$ T.V. $\left(u_{0}\right)$ and $\sum_{\mathrm{np}} \llbracket u \rrbracket=\sum\left|\theta^{\mathrm{np}}\right| \leq G\left(t_{0}\right) / 3 k$, cf. (3.38). For T.V. $\left(p^{\delta}\left(\cdot, t_{n}\right)\right)$ we find

$$
\begin{aligned}
\mathrm{T} . \mathrm{V} \cdot\left(p^{\delta}\left(\cdot, t_{n}\right)\right) & =\sum_{\mathrm{rf}} \llbracket p \rrbracket+\sum_{\text {shock }} \llbracket p \rrbracket \leq k\left(\sum_{\mathrm{rf}} \llbracket u \rrbracket+\sum_{\text {shock }} \llbracket u \rrbracket\right) \\
& \leq k \mathrm{~T} \cdot \mathrm{V} \cdot\left(u^{\delta}\left(\cdot, t_{n}\right)\right) \leq \kappa k \mathrm{~T} \cdot \mathrm{V} \cdot\left(p_{0}, u_{0}\right) .
\end{aligned}
$$

This proves (3.41) because

$$
\begin{aligned}
\mathrm{T} . \mathrm{V} \cdot\left(p^{\delta}\left(\cdot, t_{n}\right), u^{\delta}\left(\cdot, t_{n}\right)\right) & =\mathrm{T} \cdot \mathrm{V} \cdot\left(p^{\delta}\left(\cdot, t_{n}\right)\right)+\mathrm{T} \cdot \mathrm{V} \cdot\left(u^{\delta}\left(\cdot, t_{n}\right)\right) \\
& \leq 2 \kappa k \mathrm{~T} \cdot \mathrm{V} \cdot\left(p_{0}, u_{0}\right) .
\end{aligned}
$$

We also have to bound the approximate solution away from vacuum. From

$$
\sup (y) \leq|y(\infty)|+|y(-\infty)|+\text { T.V. }(y),
$$

and the fact that $p^{\delta}( \pm \infty, \cdot)=p_{0}( \pm \infty):=p_{ \pm}$, it follows that

$$
\begin{aligned}
\sup \left(p^{\delta}-p_{+}\right) & \left.\leq\left|p^{\delta}(\infty)-p_{+}\right|+\mid p^{\delta}(-\infty)-p_{+}\right) \mid+\mathrm{T} . \mathrm{V} \cdot\left(p^{\delta}\right) \\
& \left.=\mid p_{+}-p_{-}\right) \mid+\mathrm{T} . \mathrm{V} \cdot\left(p^{\delta}\right) \\
& \leq 2 \mathrm{~T} . \mathrm{V} \cdot\left(p^{\delta}\right) \leq 2 \kappa k \mathrm{~T} . \mathrm{V} \cdot\left(p_{0}, u_{0}\right) .
\end{aligned}
$$

Similarly, we obtain

$$
\begin{aligned}
& \sup \left(p^{\delta}-p_{-}\right) \leq 2 \kappa k \mathrm{~T} . \mathrm{V} \cdot\left(p_{0}, u_{0}\right) \\
& \sup \left(u^{\delta}-u_{+}\right) \leq 2 \kappa \mathrm{T} . \mathrm{V} \cdot\left(p_{0}, u_{0}\right), \\
& \sup \left(u^{\delta}-u_{-}\right) \leq 2 \kappa \mathrm{T} . \mathrm{V} \cdot\left(p_{0}, u_{0}\right) .
\end{aligned}
$$

Furthermore, $\gamma^{\delta}(\cdot, t)$ always lies between 1 and $\bar{\gamma}$. Thus, the approximate solution obtained by front tracking will always be contained in the domain

$$
\begin{aligned}
\mathcal{U}=\{(p, u, \gamma) \mid & \max \left\{\left|p-p_{-}\right|,\left|p-p_{+}\right|\right\} \leq 2 \kappa k \mathrm{~T} \cdot \mathrm{V} \cdot\left(p_{0}, u_{0}\right), \\
& \left.\max \left\{\left|u-u_{-}\right|,\left|u-u_{+}\right|\right\} \leq 2 \kappa \mathrm{T} \cdot \mathrm{V} \cdot\left(p_{0}, u_{0}\right), \gamma \in(1, \bar{\gamma}]\right\},
\end{aligned}
$$

where $p_{ \pm}=p_{0}( \pm \infty)$ and $u_{ \pm}=u_{0}( \pm \infty)$. We are now able to bound $U^{\delta}$ away from vacuum.

Lemma 3.19. If the initial data satisfy

$$
2 \kappa k \mathrm{~T} . \mathrm{V} \cdot\left(p_{0}, u_{0}\right) \leq \tilde{p}-p_{\min }
$$

for $a p_{\min }>0$ and $\tilde{p}=\max \left\{p_{-}, p_{+}\right\}$, or the stronger condition

$$
(\bar{\gamma}-1) \mathrm{T} . \mathrm{V} \cdot\left(p_{0}, u_{0}\right) \leq C_{3},
$$

where

$$
C_{3}:=\frac{\bar{\gamma}^{1 / 2}}{\kappa k r_{\max }^{\prime}}\left(\tilde{p}^{(\bar{\gamma}-1) /(2 \bar{\gamma})}-p_{\min }^{(\bar{\gamma}-1) /(2 \bar{\gamma})}\right),
$$

then $p \geq p_{\min }$ for all $U \in \mathcal{U}$. Moreover, the approximate solution obtained using front tracking is bounded and, in particular, satisfies $0<p_{\min } \leq p^{\delta}(x, t) \leq p_{\max }$. 
Proof. For a $p<\min \left(p_{0}(x)\right)$ we have

$$
\max \{|p-p(\infty, \cdot)|,|p-p(-\infty, \cdot)|\}=\max \left\{p_{-}, p_{+}\right\}-p=\tilde{p}-p,
$$

hence, $p$ is in $\mathcal{U}$ if $\tilde{p}-p \leq 2 \kappa k \mathrm{~T}$.V. $\left(p_{0}, u_{0}\right)$. Thus, if

$$
2 \kappa k \mathrm{~T} . \mathrm{V} \cdot\left(p_{0}, u_{0}\right) \leq \tilde{p}-p_{\min },
$$

for a given $p_{\min }$ so that $0<p_{\min } \leq \min \left(p_{0}\right)$, then $p \geq p_{\min }$ for all $U \in \mathcal{U}$.

Since condition 3.40 imposes a restriction on $(\bar{\gamma}-1)$ T.V. $\left(p_{0}, u_{0}\right)$, we reformulate condition 3.45 to do the same. For a $p_{*} \geq \tilde{p} \geq p_{\min }$ there is a constant $u_{*}$ so that we can write

$$
u(p)=u_{*}-r\left(p, p_{*}, \bar{\gamma}\right)
$$

From the mean value theorem we get that

$$
\left|\tilde{p}-p_{\min }\right|=\frac{1}{\left|u^{\prime}(\hat{p})\right|}\left|u(\tilde{p})-u\left(p_{\min }\right)\right| \geq \frac{1}{r_{\max }^{\prime}}\left(u\left(p_{\min }\right)-u(\tilde{p})\right),
$$

for $p_{\min } \leq \hat{p} \leq \tilde{p}$. Furthermore,

$$
\begin{aligned}
u\left(p_{\min }\right)-u(\tilde{p}) & =u_{*}-\frac{2 \bar{\gamma}^{\frac{1}{2}}}{\bar{\gamma}-1}\left(p_{\min }^{\frac{\bar{\gamma}-1}{2 \bar{\gamma}}}-p_{*}^{\frac{\bar{\gamma}-1}{2 \bar{\gamma}}}\right)-u_{*}+\frac{2 \bar{\gamma}^{\frac{1}{2}}}{\bar{\gamma}-1}\left(\tilde{p}^{\frac{\bar{\gamma}-1}{2 \bar{\gamma}}}-p_{*}^{\frac{\bar{\gamma}-1}{2 \bar{\gamma}}}\right) \\
& =\frac{2 \bar{\gamma}^{\frac{1}{2}}}{\bar{\gamma}-1}\left(\tilde{p}^{\frac{\bar{\gamma}-1}{2 \bar{\gamma}}}-p_{\min }^{\frac{\bar{\gamma}-1}{2 \bar{\gamma}}}\right)
\end{aligned}
$$

so that

$$
\tilde{p}-p_{\min } \geq \frac{2 \bar{\gamma}^{1 / 2}}{(\bar{\gamma}-1) r_{\max }^{\prime}}\left(\tilde{p}^{\frac{\bar{\gamma}-1}{2 \bar{\gamma}}}-p_{\min }^{\frac{\bar{\gamma}-1}{2 \bar{\gamma}}}\right) .
$$

Therefore, we have that $p \geq p_{\min }>0$ for all $p \in \mathcal{U}$ if

$$
2 \kappa k \mathrm{~T} . \mathrm{V} \cdot\left(p_{0}, u_{0}\right) \leq \frac{2 \bar{\gamma}^{1 / 2}}{(\bar{\gamma}-1) r_{\max }^{\prime}}\left(\tilde{p}^{\frac{\bar{\gamma}-1}{2 \bar{\gamma}}}-p_{\min }^{\frac{\bar{\gamma}-1}{2 \bar{\gamma}}}\right),
$$

which proves the lemma.

3.6. Convergence to a weak solution. The approximate solution, $U^{\delta}$, is bounded and, in particular, bounded away from vacuum. Furthermore, the total variation of $U^{\delta}$ is bounded independent of $\delta$, as shown in the previous section. Since $v=p^{-1 / \gamma}$, we furthermore have that $v^{\delta}$ is bounded and have bounded total variation independent of $\delta$. Thus, the approximate solution given in the conservative variables, $\tilde{U}^{\delta}=\left(v^{\delta}, u^{\delta}, \gamma^{\delta}\right)$, is bounded, and

$$
\mathrm{T} . \mathrm{V} .\left(\tilde{U}^{\delta}(\cdot, t)\right) \leq M_{0},
$$

for a constant $M_{0}$ independent of $\delta$.

We first use Kolmogorov's compactness theorem [15, Thm. A.5] to show that there is a subsequence of $\left\{\tilde{U}^{\delta}\right\}_{\delta>0}$ that converges in $L_{\text {loc }}^{1}(\mathbb{R} \times[0, T])$. To that end we observe that

$$
\int_{\mathbb{R}}\left|\tilde{U}^{\delta}(x+\omega, t)-\tilde{U}^{\delta}(x, t)\right| \mathrm{d} x \leq \omega \mathrm{T} . \mathrm{V} \cdot\left(\tilde{U}^{\delta}(\cdot, t)\right) \leq M_{0} \omega .
$$

Thus, it remains to show that for any $R>0$,

$$
\int_{-R}^{R}\left|\tilde{U}^{\delta}(x, t)-\tilde{U}^{\delta}(x, s)\right| \mathrm{d} x \leq M_{1}(t-s),
$$

where $t \geq s \geq 0$ and $M_{1}$ is independent of $\delta$. See Theorem A.8 in [15] for a detailed proof of why (3.48)-3.50 yield a convergent subsequence using Kolmogorov's compactness theorem. Here we proceed by showing that 3.50 holds for our system. 
For $t \in\left(\tau_{j}, \tau_{j+1}\right]$, where $\tau_{j}$ and $\tau_{j+1}$ are two consecutive collision times, we can write $\tilde{U}^{\delta}$ as

$$
\tilde{U}^{\delta}(x, t)=\sum_{k=1}^{N_{\tau_{j}}}\left(U_{k-1}^{j}-U_{k}^{j}\right) H\left(x-x_{k}^{j}(t)\right)+U_{N \tau_{j}},
$$

where $x_{k}^{j}$ is the position of the $k$ th front from the left, $H$ is the Heaviside function, $N_{\tau_{j}}$ is the number of fronts after the collision at $\tau_{j}$, and $\tilde{U}^{\delta}(x, t)=U_{k}^{j}$ for $x \in$ $\left(x_{k}^{j}, x_{k+1}^{j}\right)$. Assume now that $t \in\left[\tau_{j}, \tau_{j+1}\right]$ and $s \in\left[\tau_{j}, \tau_{j+1}\right]$ where $j \leq i$ and $s \leq t$. Then

$$
\begin{array}{rl}
\int_{\mathbb{R}}\left|\tilde{U}^{\delta}(x, t)-\tilde{U}^{\delta}\left(x, \tau_{j}\right)\right| \mathrm{d} & x=\int_{\mathbb{R}}\left|\int_{\tau_{j}}^{t} \frac{\mathrm{d}}{\mathrm{d} \hat{t}} \tilde{U}^{\delta}(x, \hat{t}) \mathrm{d} \hat{t}\right| \mathrm{d} x \\
\leq & \int_{\mathbb{R}} \int_{\tau_{j}}^{t} \sum_{k=1}^{N_{\tau_{j}}}\left|U_{k-1}^{j}-U_{k}^{j}\right|\left|\frac{\mathrm{d}}{\mathrm{d} \hat{t}} x_{k}^{j}(\hat{t})\right|\left|H^{\prime}\left(x-x_{k}^{j}(\hat{t})\right)\right| \mathrm{d} \hat{t} \mathrm{~d} x \\
& \leq \lambda_{\mathrm{np}} \int_{\tau_{j}}^{t} \sum_{k=1}^{N_{\tau_{j}}}\left|U_{k-1}^{j}-U_{k}^{j}\right| \int_{\mathbb{R}}\left|H^{\prime}\left(x-x_{k}^{j}(\hat{t})\right)\right| \mathrm{d} x \mathrm{~d} \hat{t} \\
& \leq \lambda_{\mathrm{np}}\left(t-\tau_{j}\right) \mathrm{T} \cdot \mathrm{V} \cdot\left(\tilde{U}^{\delta}(\cdot, t)\right) \leq \lambda_{\mathrm{np}} M_{0}\left(t-\tau_{j}\right),
\end{array}
$$

where we have used that $\left|\frac{\mathrm{d}}{\mathrm{d} t} x_{k}^{j}(\hat{t})\right| \leq \lambda_{\mathrm{np}}$. By similar arguments we get

$$
\begin{gathered}
\int_{\mathbb{R}}\left|\tilde{U}^{\delta}\left(x, \tau_{j}\right)-\tilde{U}^{\delta}\left(x, \tau_{j+1}\right)\right| \mathrm{d} x \leq \lambda_{\mathrm{np}} M_{0}\left(\tau_{j}-\tau_{j+1}\right), \quad \text { if } j+1<i, \\
\int_{\mathbb{R}}\left|\tilde{U}^{\delta}\left(x, \tau_{j+1}\right)-\tilde{U}^{\delta}(x, s)\right| \mathrm{d} x \leq \lambda_{\mathrm{np}} M_{0}\left(\tau_{j+1}-s\right) .
\end{gathered}
$$

Hence,

$$
\begin{aligned}
& \int_{\mathbb{R}}\left|\tilde{U}^{\delta}(x, t)-\tilde{U}^{\delta}(x, s)\right| \mathrm{d} x \leq \int_{\mathbb{R}}\left|\tilde{U}^{\delta}(x, t)-\tilde{U}^{\delta}\left(x, \tau_{j}\right)\right| \mathrm{d} x \\
& \quad+\int_{\mathbb{R}}\left|\tilde{U}^{\delta}\left(x, \tau_{j}\right)-\tilde{U}^{\delta}\left(x, \tau_{j+1}\right)\right| \mathrm{d} x+\int_{\mathbb{R}}\left|\tilde{U}^{\delta}\left(x, \tau_{j+1}\right)-\tilde{U}^{\delta}(x, s)\right| \mathrm{d} x \\
& \quad \leq \lambda_{\mathrm{np}} M_{0}(t-s),
\end{aligned}
$$

where the middle integral is only included if $j+1<i$. Since $\lambda_{\mathrm{np}} M_{0}$ is a constant independent of $\delta$, we have now established 3.50. Hence, there exists a function $U(x, t)$ and a subsequence $\left\{\delta_{j}\right\} \subset\{\delta\}$ so that $U^{\delta_{j}} \rightarrow U$ in $L_{\text {loc }}^{1}(\mathbb{R} \times[0, T])$ as $j \rightarrow \infty$.

We still have to show that the limit is a weak solution. Recall from equation (2.1) that $U=(v, u, \gamma)$ is a weak solution on a strip $[t, s]$ if

$$
\begin{aligned}
\mathcal{I}_{t}^{s}(U):= & \int_{t}^{s} \int_{\mathbb{R}} U \phi_{t}+f(U) \phi_{x} \mathrm{~d} x \mathrm{~d} t \\
& -\int_{\mathbb{R}} U(x, s) \phi(x, s) \mathrm{d} x+\int_{\mathbb{R}} U(x, t) \phi(x, t) \mathrm{d} x=0,
\end{aligned}
$$

for all test functions $\phi$. Fix two successive collision times, $\tau_{j}$ and $\tau_{j+1}$, and let $\tilde{U}^{\delta}$ be the approximate solution found using front tracking. The approximate solution $\tilde{U}^{\delta}$ is not a weak solution because we have introduced non-physical fronts and approximate rarefaction waves. Therefore, we need to estimate how far $\tilde{U}^{\delta}$ is from the weak solution.

Let $s_{1}=\tau_{j}$ and let $V_{i}(x, s)$ be the weak solution of

$$
V_{t}+f(V)_{x}=0, \quad V\left(x, s_{i}\right)=\tilde{U}^{\delta}\left(x, s_{i}\right) .
$$


We find $V_{1}$ fot $t$ close to $s_{1}$ by solving exactly the Riemann problems at the jumps of $\tilde{U}^{\delta}\left(x, s_{1}\right)$. This solution is defined up to the time $s_{2}>s_{1}$ when the first waves interact. If no waves in $V_{1}$ collide before $\tau_{j+1}$, we have $s_{2}=\tau_{j+1}$. Otherwise, we let $V_{2}$ be the solution for $s \geq s_{2}$ of $\left(3.52\right.$ with $i=2$ and $\tilde{U}^{\delta}\left(x, s_{2}\right)$ as initial data. In this way we fill $\left[\tau_{j}, \tau_{j+1}\right)$ with small strips $\left[s_{i}, s_{i+1}\right)$ on which we have defined $V_{i}$. Let $V$ denote the function that equals $V_{i}$ at each interval $\left[s_{i}, s_{i+1}\right)$, thus, $V\left(x, s_{i}\right)=\tilde{U}^{\delta}\left(x, s_{i}\right)$ for each $i$. Figure 18(a) shows this construction when a non-physical front is present in $\tilde{U}^{\delta}$, while Figure $18(\mathrm{~b})$ shows the first steps in the construction when a rarefaction front interacts with a front of the same family at $\tau_{j+1}$.

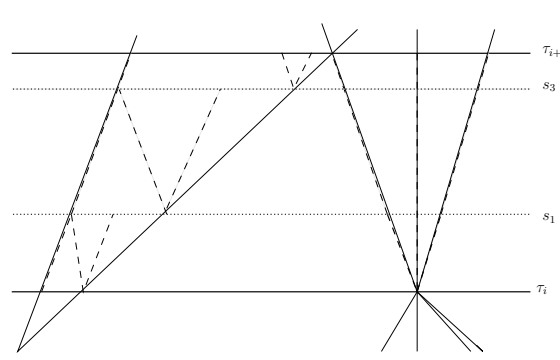

(a) When $\tilde{U}^{\delta}$ contains a non-physical front

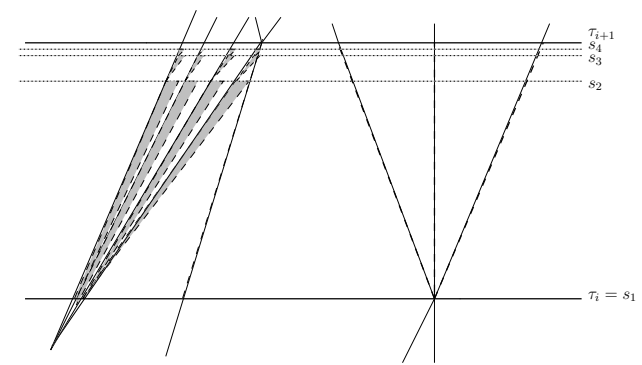

(b) The first steps when a rarefaction front interacts with a front of the same family at $\tau_{j+1}$.

Figure 18. The approximate solution $\tilde{U}^{\delta}$ (solid lines) and the exact solution $V_{i}$ (dashed line) at each interval $\left[s_{i}, s_{i+1}\right)$.

Let furthermore $V^{\delta}$ be the approximation of $V$ found by solving (3.52) using front tracking without non-physical fronts. That is, we solve all Riemann problems using the approximate solver and never use the simplified solver. No front in $V^{\delta}$ will interact in one strip, thus, $V^{\delta}$ only differs from $V$ for rarefaction waves. From the approximation of rarefaction waves by fronts, we have that $\left|V_{i}(x, t)-V^{\delta}(x, t)\right|=$ $\mathcal{O}(\delta)$ for $(x, t)$ in a rarefaction fan. Thus, for $t \in\left[s_{i}, s_{i+1}\right]$ the integral

$$
\int_{\mathbb{R}}\left|V(x, t)-V^{\delta}(x, t)\right| \mathrm{d} x
$$

will be the sum of the integrals across the rarefaction fans of $V$. Let $V_{l}$ and $V_{r}$ be the left and right state of such a fan. The integral over this fan will be the sum of the integrals across each step in $V^{\delta}$, and there are $\left|p_{r}-p_{l}\right| / \mathcal{O}(\delta)$ steps, each with the width $\left(t-s_{i}\right) \Delta \lambda=\left(t-s_{i}\right) \mathcal{O}(\delta)$, where $\Delta \lambda$ is the difference in the characteristic speed across a rarefaction-front in $V^{\delta}$. We sum over $j$, that is, over all rarefaction fans in $V$, and find that

$$
\begin{aligned}
\int_{\mathbb{R}}\left|V(x, t)-V^{\delta}(x, t)\right| \mathrm{d} x & =\sum_{j} \frac{\left|p_{r}^{j}-p_{l}^{j}\right|}{\mathcal{O}(\delta)} \mathcal{O}(\delta)\left(t-s_{i}\right) \mathcal{O}(\delta) \\
& \leq\left(t-s_{i}\right) \mathrm{T} \cdot \mathrm{V} \cdot\left(p^{\delta}\right) \mathcal{O}(\delta) \leq\left(t-s_{i}\right) \mathcal{O}(\delta)
\end{aligned}
$$

since $\sum_{j}\left|p_{r}^{j}-p_{l}^{j}\right| \leq \mathrm{T} \cdot \mathrm{V} \cdot\left(p^{\delta}\right) \leq M_{0}$

Next, we compare $V^{\delta}$ to $U^{\delta}$. Since $U^{\delta}\left(x, s_{i}\right)$ is used as initial data solving (3.52) for each strip, the two solutions only differs where $U^{\delta}$ have Riemann problems 
solved by the simplified solver. Thus, in order to estimate the integral

$$
\int_{\mathbb{R}}\left|U^{\delta}(x, t)-V^{\delta}(x, t)\right| \mathrm{d} x,
$$

for a $t \in\left[s_{i}, s_{i+1}\right)$, we need to take a closer look at the approximation done using the simplified Riemann solver defined in Section 3.1. Consider the interaction $\zeta+\sum_{i}^{n} \epsilon_{i}$ and note that the arguments are similar for the symmetric case. As in Lemma 3.1, we let $\hat{\epsilon}+\zeta+\hat{\eta}$ with intermediate states $\hat{U}_{i}, i=1,2$, be the solution using the approximate solver, and $\epsilon^{\prime}+\zeta+\theta^{\text {np }}$ and $\epsilon^{\prime}+\zeta+\eta^{\prime}+\theta^{\text {np }}$ with intermediate states $\tilde{U}_{i}, i=1,2$ and $i=1,2,3$, be the solution using the simplified solver for $n=1$ and $n>1$, respectively. If $\epsilon^{\prime}$ is a shock-front, then $\alpha^{\prime}$ and $\hat{\alpha}$ have slightly different speeds giving rise to a jump in $\left|U^{\delta}-V^{\delta}\right|$ of the width $\left|\sigma_{\hat{\alpha}}-\sigma_{\alpha^{\prime}}\right|\left(t-s_{i}\right)=$ $\mathcal{O}(1)\left|\theta^{\text {np }}\right|\left(t-s_{i}\right)$, cf. Lemma 3.1. If $\epsilon^{\prime}$ is a rarefaction-front, the speeds are equal. If $n>1$ and $\hat{\eta}$ is of the same type as $\eta^{\prime}$, we also get a jump in $\left|U^{\delta}-V^{\delta}\right|$ due to different speeds. The width of this jump is $\left|\sigma_{\hat{\eta}}-\sigma_{\eta^{\prime}}\right|\left(t-s_{i}\right)=\mathcal{O}(1)\left|\theta^{\text {np }}\right|\left(t-s_{i}\right)$, where we let $\sigma$ denote the speed for both shock- and rarefaction-fronts. If $\hat{\eta}$ and $\eta^{\prime}$ are of different types, the height of the jump due to different speeds is less than $\left|U_{r}-\tilde{U}_{3}\right|$. The remaining jumps in $\left|U^{\delta}-V^{\delta}\right|$ over this interaction are $\left|\hat{U}_{1}-\tilde{U}_{1}\right|$, $\left|\hat{U}_{2}-\tilde{U}_{2}\right|$, and $\left|U_{r}-\tilde{U}_{j}\right|$ where $j=2$ if $n=1$, and $j=3$ if $n>1$. From Lemma 3.1 we have that the heights of these jumps are all bounded by $\mathcal{O}(1)\left|\theta^{\mathrm{np}}\right|$. Thus,

$$
\int_{\mathbb{R}}\left|U^{\delta}(x, t)-V^{\delta}(x, t)\right| \mathrm{d} x \leq \sum_{\mathrm{np}} \mathcal{O}(1)\left|\theta^{\mathrm{np}}\right|\left(t-s_{i}\right)=\mathcal{O}(\delta)\left(t-s_{i}\right),
$$

using that $\sum\left|\theta^{\text {np }}\right| \leq \mathcal{O}(1) \delta_{\text {init }}=\mathcal{O}(\delta)$ according to Lemma 3.17

Combining 3.53 and 3.55 , we finally get

$$
\begin{aligned}
\int_{\mathbb{R}}\left|U^{\delta}(x, t)-V(x, t)\right| \mathrm{d} x & \leq \int_{\mathbb{R}}\left|U^{\delta}(x, t)-V^{\delta}(x, t)\right|+\int_{\mathbb{R}}\left|V(x, t)-V^{\delta}(x, t)\right| \\
& =\mathcal{O}(\delta)\left(t-s_{i}\right) .
\end{aligned}
$$

Our goal is to show that $\left|\mathcal{I}_{0}^{T}(U)\right|=0$ where $U(x, t)$ is the limit of $\tilde{U}^{\delta_{j}}(x, t)$. We start by estimating $\left|\mathcal{I}_{s_{i}}^{s_{i+1}}\left(\tilde{U}^{\delta}\right)\right|$. Recall that $V_{i}\left(x, s_{i}\right)=\tilde{U}^{\delta}\left(x, s_{i}\right)$ and that $V_{i}$ is a weak solution on each strip, thus, $\mathcal{I}_{s_{i}}^{s_{i+1}}\left(V_{i}\right)=0$. We start with $v^{\delta}$;

$$
\begin{aligned}
&\left|\mathcal{I}_{s_{i}}^{s_{i+1}}\left(v^{\delta}\right)\right|=\left|\mathcal{I}_{s_{i}}^{s_{i+1}}\left(v^{\delta}\right)-\mathcal{I}_{s_{i}}^{s_{i+1}}\left(v_{i}\right)\right| \\
&=\mid \int_{s_{i}}^{s_{i+1}} \int_{\mathbb{R}}\left(v^{\delta}-v_{i}\right) \phi_{t}+\left(-u^{\delta}+u_{i}\right) \phi_{x} \mathrm{~d} x \mathrm{~d} t \\
& \quad-\int_{\mathbb{R}}\left(v^{\delta}\left(x, s_{i+1}\right)-v_{i}\left(x, s_{i+1}\right)\right) \phi\left(x, s_{i+1}\right) \mathrm{d} x \mid \\
& \leq M_{2}\left(\int_{s_{i}}^{s_{i+1}} \int_{\mathbb{R}}\left|v^{\delta}-v_{i}\right|+\left|u^{\delta}-u_{i}\right| \mathrm{d} x \mathrm{~d} t\right. \\
&\left.\quad+\int_{\mathbb{R}}\left|v^{\delta}\left(x, s_{i+1}\right)-v_{i}\left(x, s_{i+1}\right)\right| \mathrm{d} x\right) \\
& \leq \mathcal{O}(\delta)\left(\left(s_{i+1}-s_{i}\right)^{2}+\left(s_{i+1}-s_{i}\right)\right)
\end{aligned}
$$

where $M_{2}$ bounds $\left|\phi_{x}\right|$ and $\left|\phi_{t}\right|$. For $u^{\delta}$ we get

$$
\begin{aligned}
\left|\mathcal{I}_{s_{i}}^{s_{i+1}}\left(u^{\delta}\right)\right| & =\left|\mathcal{I}_{s_{i}}^{s_{i+1}}\left(u^{\delta}\right)-\mathcal{I}_{s_{i}}^{s_{i+1}}\left(u_{i}\right)\right| \\
& =\mid \int_{s_{i}}^{s_{i+1}} \int_{\mathbb{R}}\left(u^{\delta}-u_{i}\right) \phi_{t}+\left(p^{\delta}-p_{i}\right) \phi_{x} \mathrm{~d} x \mathrm{~d} t
\end{aligned}
$$




$$
\begin{aligned}
& \quad-\int_{\mathbb{R}}\left(u^{\delta}\left(x, s_{i+1}\right)-u_{i}\left(x, s_{i+1}\right)\right) \phi\left(x, s_{i+1}\right) \mathrm{d} x \mid \\
& \leq M_{2}\left(\int_{s_{i}}^{s_{i+1}} \int_{\mathbb{R}}\left|u^{\delta}-u_{i}\right|+\left|p^{\delta}-p_{i}\right| \mathrm{d} x \mathrm{~d} t\right. \\
& \left.\quad+\int_{\mathbb{R}}\left|u^{\delta}\left(x, s_{i+1}\right)-u_{i}\left(x, s_{i+1}\right)\right| \mathrm{d} x\right) \\
& \leq \mathcal{O}(\delta)\left(\left(s_{i+1}-s_{i}\right)^{2}+\left(s_{i+1}-s_{i}\right)\right),
\end{aligned}
$$

where we have used that

$$
\int_{\mathbb{R}}\left|p_{i}(x, t)-p^{\delta}(x, t)\right| \mathrm{d} x \leq\left(t-s_{i}\right) \mathcal{O}(\delta),
$$

by the same arguments as above. Since $\gamma$ only changes along contact discontinuities and these are solved exactly both by the approximate and the simplified solver, we actually have $\gamma_{i}(x, t)=\gamma^{\delta}(x, t)$, thus

$$
\left|\mathcal{I}_{s_{i}}^{s_{i+1}}\left(\gamma^{\delta}\right)\right|=\left|\mathcal{I}_{s_{i}}^{s_{i+1}}\left(\gamma_{i}\right)\right|=0 .
$$

Let $\tau_{j}$ and $\tau_{j+1}$ still be two successive collision times and recall that $\tau_{j+1}-\tau_{j}=$ $\sum_{i=1}^{\infty}\left(s_{i+1}-s_{i}\right)$. Thus,

$$
\begin{aligned}
\left|\mathcal{I}_{\tau_{j}}^{\tau_{j+1}}\left(\tilde{U}^{\delta}\right)\right| & \leq \sum_{i=1}^{\infty}\left|\mathcal{I}_{s_{i}}^{s_{i+1}}\left(\tilde{U}^{\delta}\right)\right| \\
& \leq \sum_{i=1}^{\infty} \mathcal{O}(\delta)\left(\left(s_{i+1}-s_{i}\right)^{2}+\left(s_{i+1}-s_{i}\right)\right) \\
& \leq \mathcal{O}(\delta)\left(\left(\tau_{j+1}-\tau_{j}\right)^{2}+\left(\tau_{j+1}-\tau_{j}\right)\right) .
\end{aligned}
$$

Since $\tilde{U}^{\delta}$ is bounded and $\tilde{U}^{\delta_{j}}(x, t) \rightarrow U(x, t)$ in $L_{\text {loc }}^{1}$ where $U=(v, u, \gamma), p^{\delta}$ will converge to $p=v^{-1 / \gamma}$ in $L_{\text {loc }}^{1}$. Thus, for any time $T<\infty$,

$$
\begin{aligned}
\left|\mathcal{I}_{0}^{T}(U)\right| & =\lim _{\delta \rightarrow 0}\left|\mathcal{I}_{0}^{T}\left(\tilde{U}^{\delta}\right)\right|=\lim _{\delta \rightarrow 0} \sum_{j}\left|\mathcal{I}_{\tau_{j}^{j+1}}^{\tau_{j}}\left(\tilde{U}^{\delta}\right)\right| \\
& =\lim _{\delta \rightarrow 0} \sum_{j} \mathcal{O}(\delta)\left(\left(\tau_{j+1}-\tau_{j}\right)^{2}+\left(\tau_{j+1}-\tau_{j}\right)\right) \\
& \leq \lim _{\delta \rightarrow 0} \mathcal{O}(\delta)\left(T+T^{2}\right)=0,
\end{aligned}
$$

which proves that $\tilde{U}^{\delta}$ converges to a weak solution of $(1.1)$ as $\delta \rightarrow 0$.

Thus, we have finally proved the main theorem:

Theorem 3.20. Consider the Cauchy problem for system (1.1) with initial data 1.2 where $\inf \left(p_{0}(x)\right)>0$ and $1 \leq \gamma_{0}(x) \leq \bar{\gamma}$. Assume that the initial data $\left(u_{0}, p_{0}\right)$ and $\gamma(x)$ satisfy

$$
\begin{gathered}
(\bar{\gamma}-1) \text { T.V. }\left(p_{0}, u_{0}\right) \leq \min \left\{\frac{C}{9 k C_{1}}, C_{3}\right\}, \\
\text { T.V. }(\gamma(x)) \leq \frac{C}{9 C_{2}} .
\end{gathered}
$$

Then the front tracking algorithm produces a sequence of approximate solutions which converges to a global weak solution of the system (1.1).

Note that all constants only depend on $p_{\min }, p_{\max }$ and $\bar{\gamma}$. Thus, by reducing $\bar{\gamma}$, we may allow arbitrary large total variation for $p_{0}$ and $u_{0}$. 
By the results of Wagner [27, there is a one-to-one correspondence between a weak solution of (1.1) and a weak solution of the system given in Eulerian coordinates,

$$
\begin{aligned}
\rho_{t}+(\rho u)_{x} & =0 \\
(\rho u)_{t}+\left(\rho u^{2}+p(\rho, \gamma)\right)_{x} & =0 \\
(\rho \gamma)_{t}+(\rho u \gamma)_{x} & =0
\end{aligned}
$$

where $x \in \mathbb{R}$ is the physical space variable and $t \in(0, \infty)$ denotes time.

\section{NumERICAL EXAMPLES}

We have implemented an approximate and a simplified Riemann solver as described in Subsection 3.1 using MATLAB. These are used together with the fronttracking code at the web page of $\left[1^{1}\right]^{1}$. The threshold parameter $\rho$, which determines when to invoke the simplified Riemann solver, is set to $\delta^{3}$ for all examples. Furthermore, we let $\lambda_{\mathrm{np}}=2\left\lceil\lambda_{\max }\right\rceil$. The front-tracking code is slightly adjusted so that $G(t)$ is computed for all times.

We find $p_{\max }$ as described in Subsection 2.3. Instead of using (3.46) to find $p_{\min }$, we choose a suitable candidate for $p_{\min }$ and then check that this candidate indeed satisfies $p_{\min } \leq p^{\delta}(x, t)$ for all $x$ and $t$. For the two first examples

$$
p_{\min }=\min \left(p_{0}(x)\right)-\left(p_{\max }-\max \left(p_{0}(x)\right)\right),
$$

is used as our candidate.

Example 4.1. The initial data in this example are piecewise constant and symmetric. We have one gas with $p=1.26, u=3.00$ and $\gamma=1.051$ which initially is trapped by another gas with $p=1.30, u=2.99$ and $\gamma=1.010$. This is the same initial data as used in Example 1 in 16 where we solved the problem using the Glimm scheme. The constants calculated for this example are listed in Table 1 .

\begin{tabular}{|c|c|c|c|c|c|c|}
\hline$p_{\max }$ & $p_{\min }$ & $\bar{\gamma}$ & $C_{1}$ & $C_{2}$ & $C$ & $k$ \\
\hline 1.3067 & 1.2534 & 1.051 & 15.9703 & 1.3309 & 1 & 1.3309 \\
\hline
\end{tabular}

TABLE 1. The constants for Example 4.1 .

and 3.40 is satisfied since

$$
\begin{gathered}
\text { T.V. }\left(p_{0}, u_{0}\right)=0.1 \leq 0.1025=C /\left(9 k C_{1}(\bar{\gamma}-1)\right), \\
\text { T.V. }\left(\gamma_{0}\right)=0.082 \leq 0.0835=C /\left(9 C_{2}\right) .
\end{gathered}
$$

Figure 19 shows $U^{\delta}(\cdot, t)$ at some different times. In Figure 20 the solution is compared with the solution found using the Glimm scheme, cf. 16. The solution obtained using the two methods are very similar, except that the contact discontinuities move back and forth due to the randomness of the Glimm scheme whereas they in front tracking always stay at \pm 1 . Note that no non-physical fronts has been generated at this point.

The front-tracking solution in the $(x, t)$-plane is shown in Figure 21. Here $\delta=0.0005$, thus, the rarefaction fronts are very close and look like rarefaction fans. Note furthermore that one front is one line regardless of its strength, thus, in Figure 21 one does not distinguish between strong and weak shock-fronts. Therefore, Figure 21 picks up the interaction of small fronts which is very hard to do using the Glimm scheme, cf. 16. In this example we see that after some time, one non-physical front is generated, and from then on, there is no more interactions with a contact discontinuity. Finally, Figure 22 shows $G(t)$ for this example.

\footnotetext{
$1_{\text {http: }} / /$ www.math.ntnu.no/ holden/FrontBook/matlabcode.html
} 

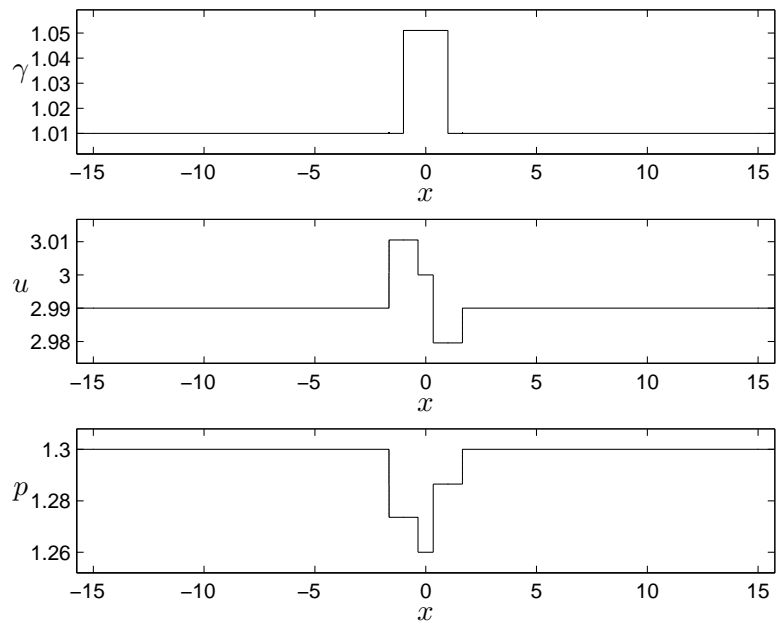

(a) $t=0.507$.
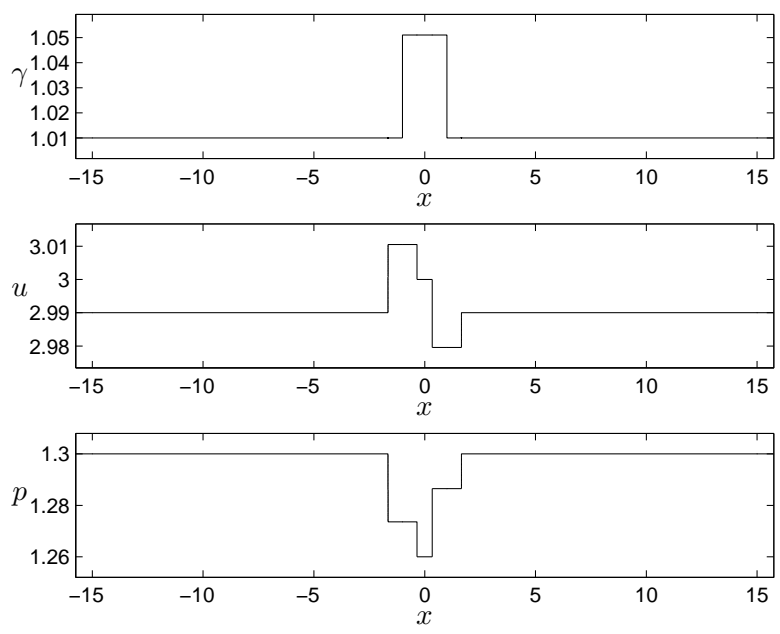

(b) $t=2.039$.
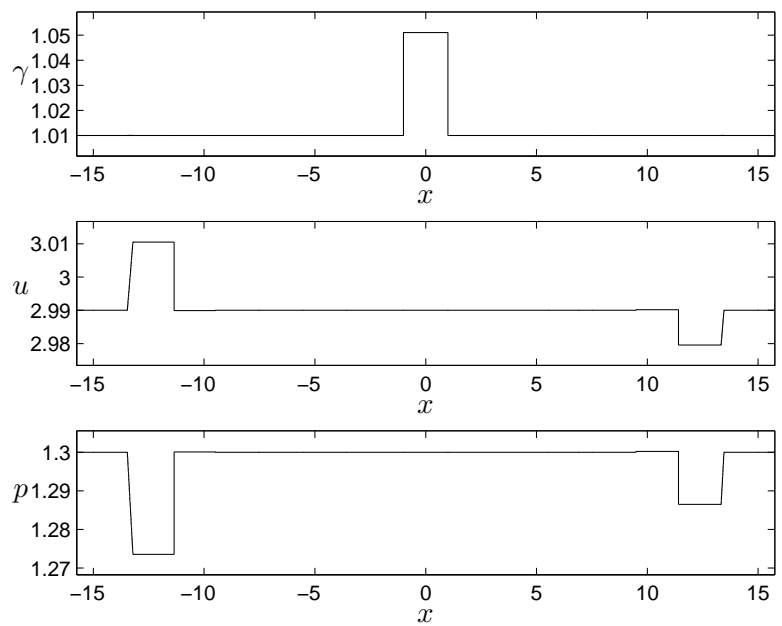

(c) $t=9.55$.

Figure 19. The solution $U^{\delta}(\cdot, t)$ at different times $t$ for Example 4.1 . 

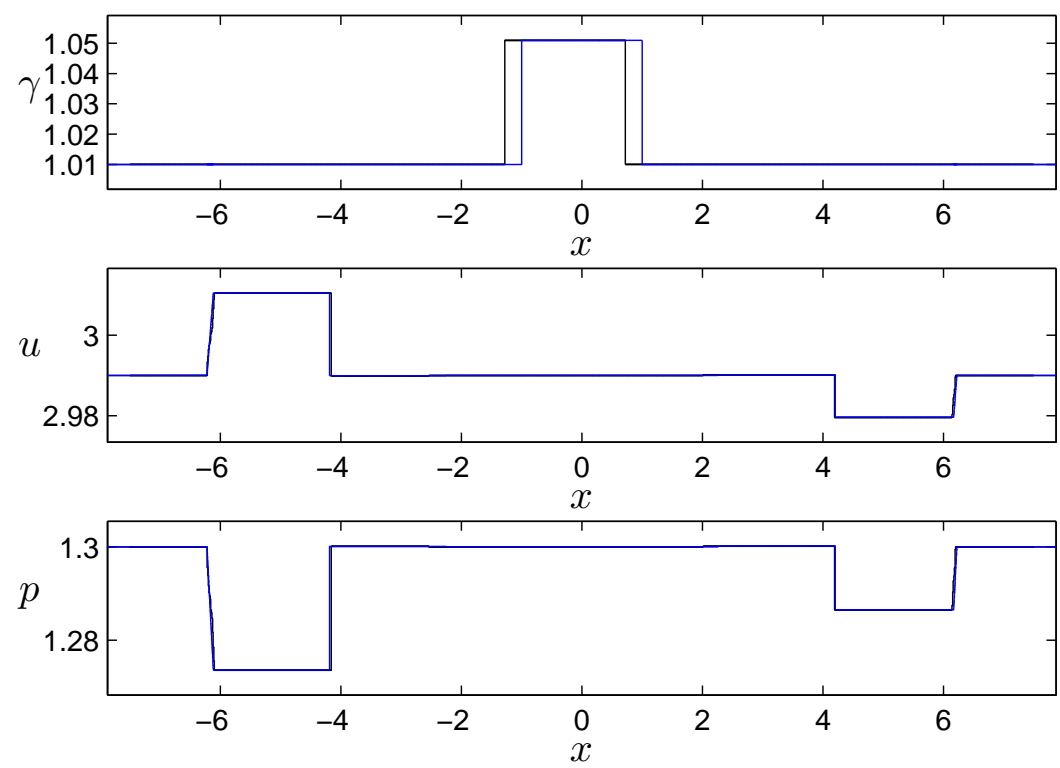

Figure 20. The solution of Example 4.1 at $t=4.002$ using front tracking (dotted line) and the Glimm scheme (solid line).

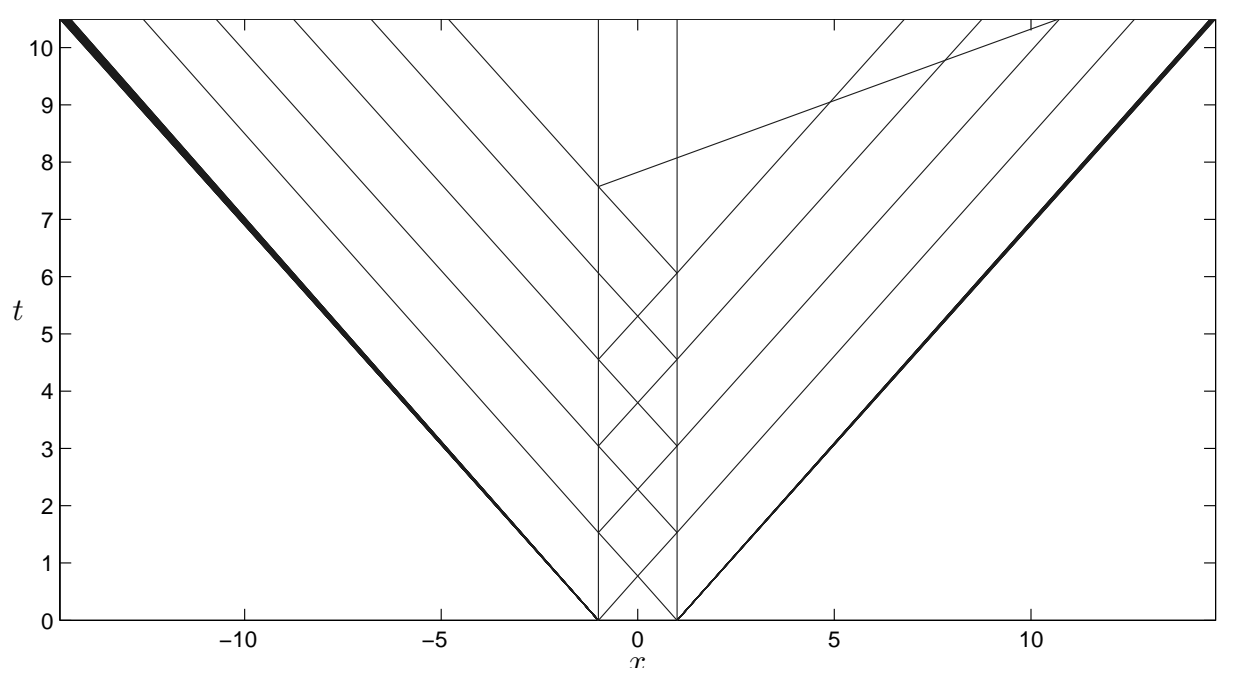

Figure 21. The solution $U^{\delta}(x, t)$ in the $(x, t)$-plane for Example 4.1

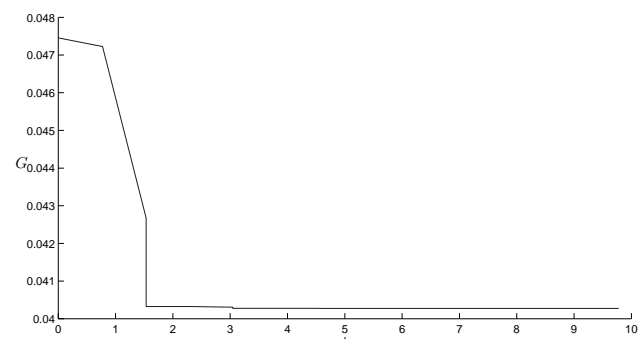

FIgURE 22. $G(t)$ for Example 4.1 . 
Example 4.2. The initial data in this example are also piecewise constant. For $-1<x<1$ we have $p=2.5$ and $u=3.0$, while we outside the unit interval have $p=1.5$ and $u=2.0$. Furthermore, $\gamma=1.5$ for $x<-1$ and $0<x<1$ while $\gamma=2.0$ for $-1<x<0$ and $x>1$.

These initial data are far from satisfying condition 3.40 , and are therefore not covered by Theorem 3.20. However, as shown in Figure 25. $G(t)$ does decrease for this example, which is enough to ensure convergence to a weak solution.

In Figure 23 we see $U^{\delta}(\cdot, t)$ at some different times, while Figure 24 shows the solution in the $(x, t)$-plane. The split rarefaction waves are more visible here, because we have used $\delta=0.1$. Initially we have three Riemann problems. The solution of the one situated at $x=-1$ is a 1 -shock wave, a contact discontinuity and a 3-rarefaction wave, the one situated at $x=1$ is almost symmetric with a solution consisting of a 1-rarefaction wave, a contact discontinuity and a 3-shock wave, while at $x=0$ we have only a jump in $\gamma$, hence, the solution is a single contact discontinuity. We see that the first non-physical fronts are generated when reflected fronts of a $\gamma$-collision interacts with another contact discontinuity. The reflected fronts become weaker for each $\gamma$-collision, thus, after some time, all fronts present between the contact discontinuities are non-physical fronts. These nonphysical fronts just pass through the contact discontinuities without generating more reflected fronts. Recall that $p$ and $\gamma$ are constant across non-physical fronts, thus, comparing the plots for $p$ and $u$ in Figure 23 we see that the non-physical fronts are small compared to the physical fronts.

Example 4.3. In this example $\gamma_{0}$ is a continuous function where $\gamma_{0}=1.7406$ for $x \leq-0.4, \gamma_{0}=2.6994$ for $x \geq 0.6$ and increases smoothly from 1.7406 to 2.6994 by a sine function in the region $-0.4 \leq x \leq 0.6$. In the same region we have a high initial pressure, $p_{0}=8$, while $p_{0}=3$ outside. The velocity is piecewise constant and decaying; $u_{0}=3$ for $x \leq-0.4, u_{0}=2$ for $-0.4 \leq x \leq 0.6$ and $u_{0}=1$ for $x \geq 0.6$. The initial data are made piecewise constant with $\delta_{\text {init }}=\Delta x=0.2$. Furthermore, we have chosen $\delta=0.2$.

These initial data are far from satisfying condition 3.40 , but $G$ is still decreasing as shown in in Figure 28. For this example we have that $p^{\delta}(x, t) \geq 3$, and therefore $p_{\text {min }}$ is set to 3 .

In Figure 26 we see $U^{\delta}(\cdot, t)$ at some different times, while Figure 27 shows the solution in the $(x, t)$-plane. In Figure 27 we observe many fronts interacting, but Figure 26 reveals that after a short time, all fronts except the leftmost and rightmost shocks are very weak fronts, including the non-physical fronts. This is also in accordance with Figure 28 where we after a short time have only very small changes in $G$.

\section{REFERENCES}

[1] D. Amadori and A. Corli. On a model of multiphase flow. arXiv:math.AP/0605057 v2 7 July 2007.

[2] F. Asakura. Wave-front tracking for the equation of non-isentropic gas dynamics. RIMS Kokyuroku, 1495:78-91, 2006.

[3] F. Asakura. Wave-front tracking for the equation of isentropic gas dynamics. Quart. Appl. Math., 63(1):20-33, 2005.

[4] A. Bressan. Hyperbolic Systems of Conservation Laws. Oxford University Press, 2000.

[5] A. Bressan and R. M. Colombo. Unique solutions of $2 \times 2$ conservation laws with large data. Indiana Univ. Math. J., 44(3):677-725, 1993.

[6] C. Chalons and F. Coquel. Navier-Stokes equations with several independent pressure laws and explicit predictor-corrector schemes. Numer. Math. 101:451-478, 2005.

[7] G.-Q. Chen, C. Christoforou, and Y. Zhang. Dependence of entropy solutions in the large for the Euler equations on nonlinear flux functions. Indiana Univ. Math. J. 56(5):2535-2567, 2007. 

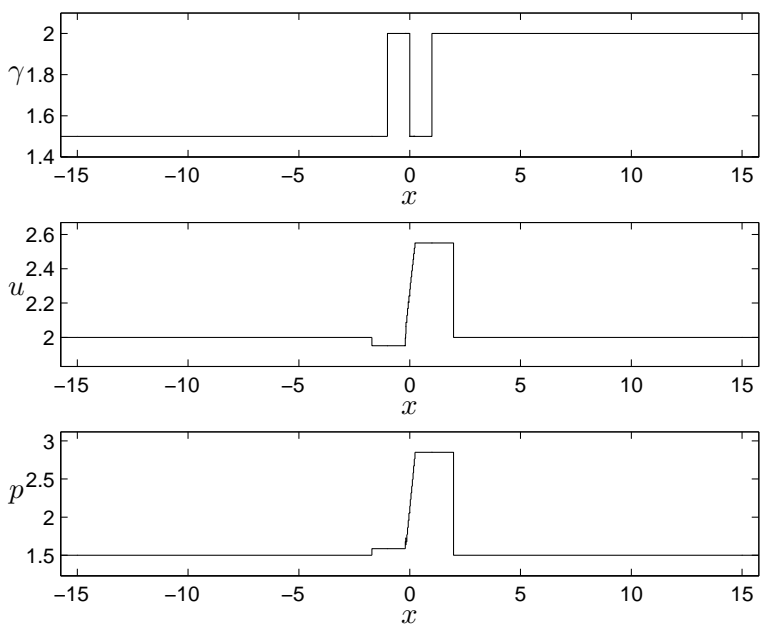

(a) $t=0.4$
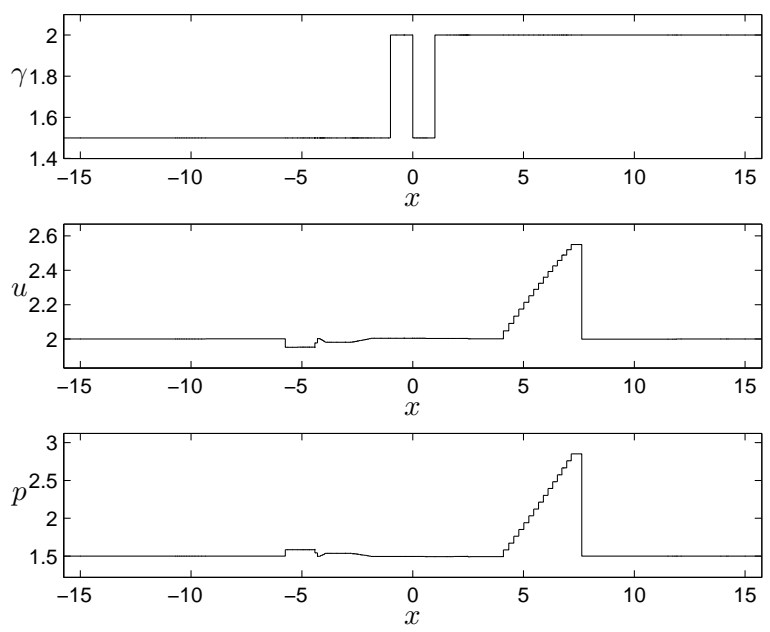

(b) $t=2.7$.
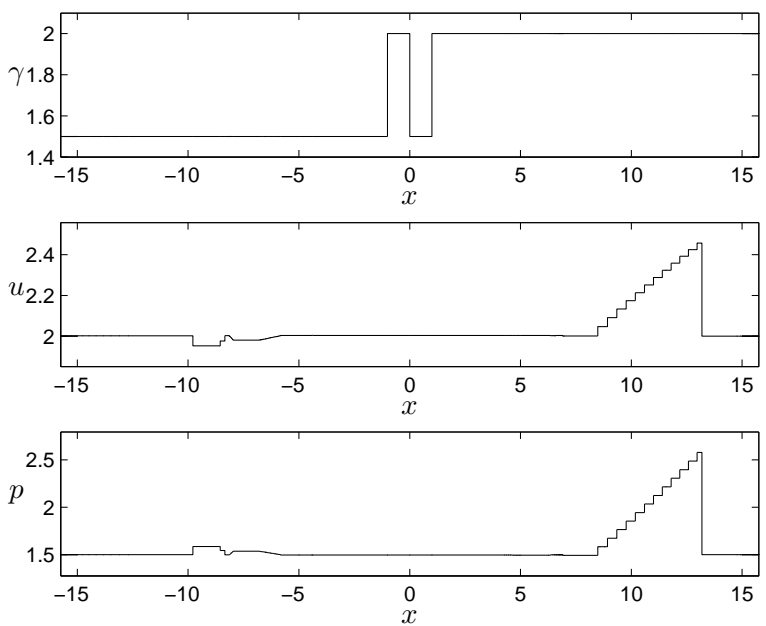

(c) $t=5$.

Figure 23. $U^{\delta}(\cdot, t)$ at different times $t$ for Example 4.2 


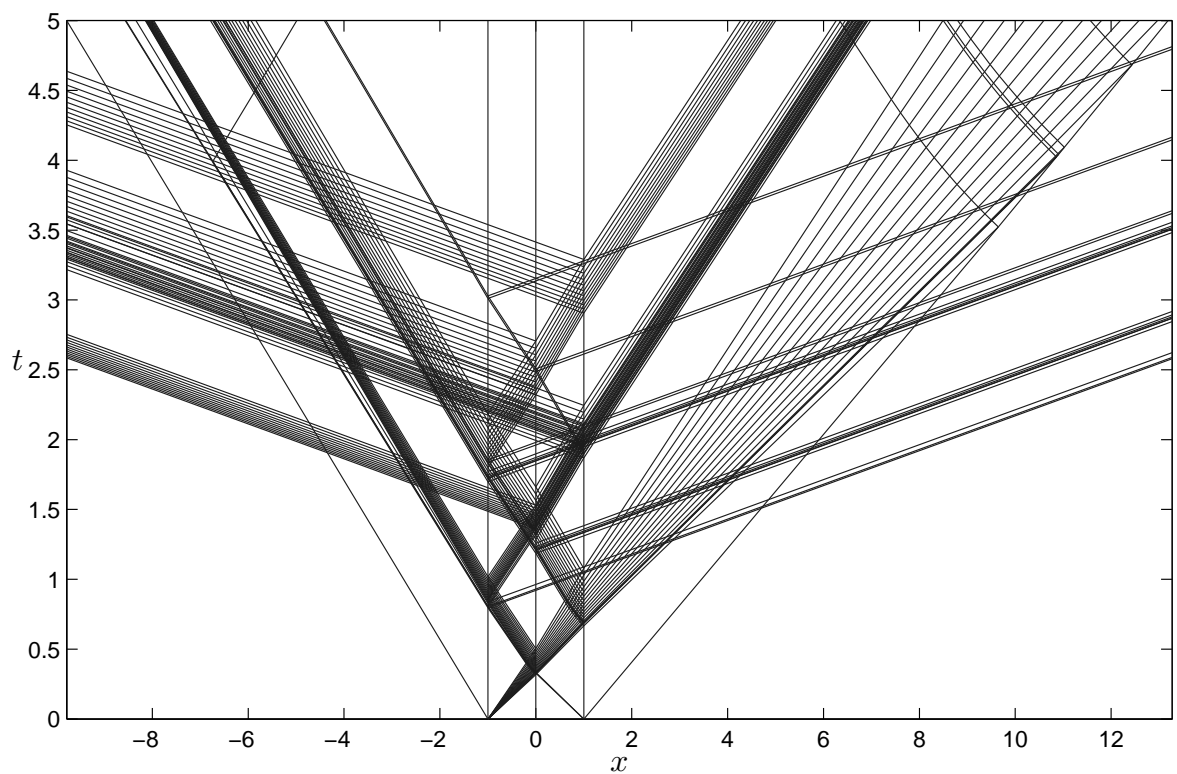

Figure 24. The solution $U^{\delta}(x, t)$ in the $(x, t)$-plane for Example 4.2

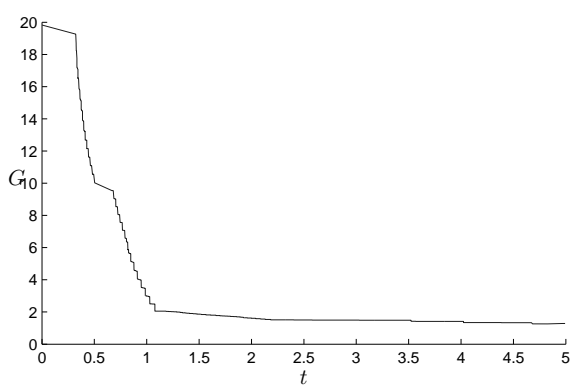

Figure 25. $G$ for Example 4.2

[8] G.-Q. Chen, C. Christoforou, and Y. Zhang. Continuous dependence of entropy solutions to the Euler equations on the adiabatic exponent and Mach number Arch. Ration. Mech. Anal. 189:97-130, 2008.

[9] R. J. DiPerna. Existence in the large for quasilinear hyperbolic conservation laws. Arch. Rational Mech. Anal., 52:244-257, 1973.

[10] R. J. DiPerna. Global existence of solutions to nonlinear hyperbolic systems of conservation laws. J. Differential Equations, 20:187-212, 1976.

[11] S. Evje and K. H. Karlsen. Global existence of weak solutions for a viscous two-phase model. J. Differential Equations, to appear.

[12] H. Fan. On a model of the dynamics of liquid/vapor phase transitions. SIAM J. Appl. Math., 60(4):1270-1301. 2000.

[13] J. Glimm. Solution in the large for nonlinear hyperbolic systems of equations. Comm. Pure Appl. Math., 18:697-715, 1965.

[14] D. Hoff. Invariant regions for systems of conservations laws. Trans. Amer. Math. Soc., 289(2):591-610, 1985.

[15] H. Holden and N. H. Risebro. Front Tracking for Hyperbolic Conservation Laws. SpringerVerlag, 2002.

[16] H. Holden, N. H. Risebro and H. Sande. The solution of the Cauchy problem with large data for a model of a mixture of gases. To appear in J. Hyperbolic Differ. Eq. 

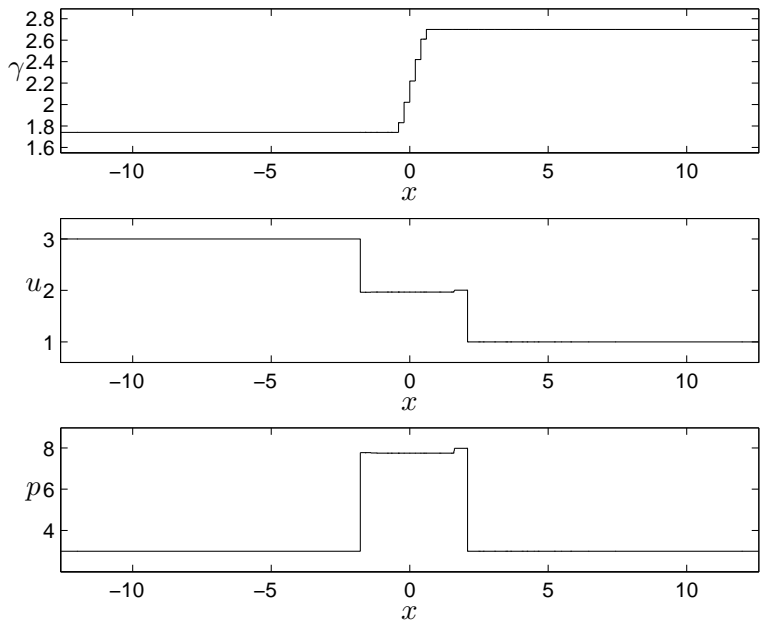

(a) $t=0.3$
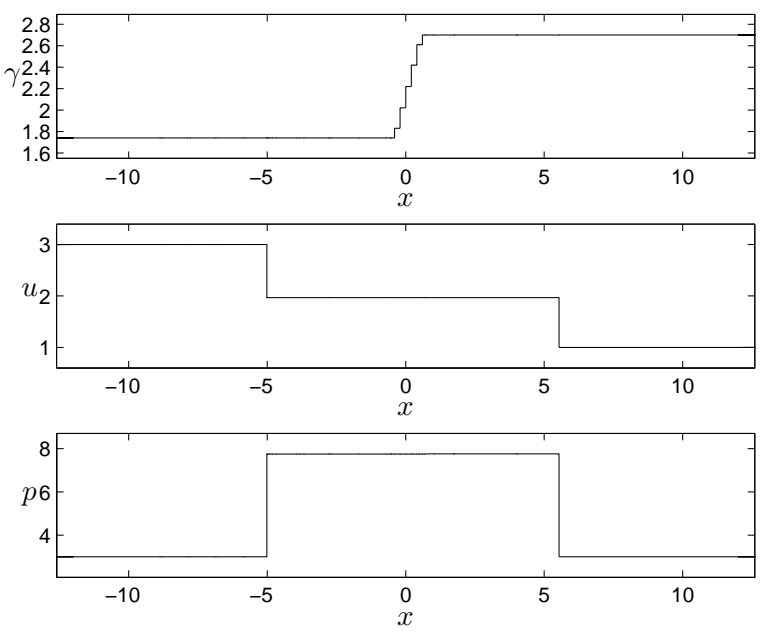

(b) $t=1$.
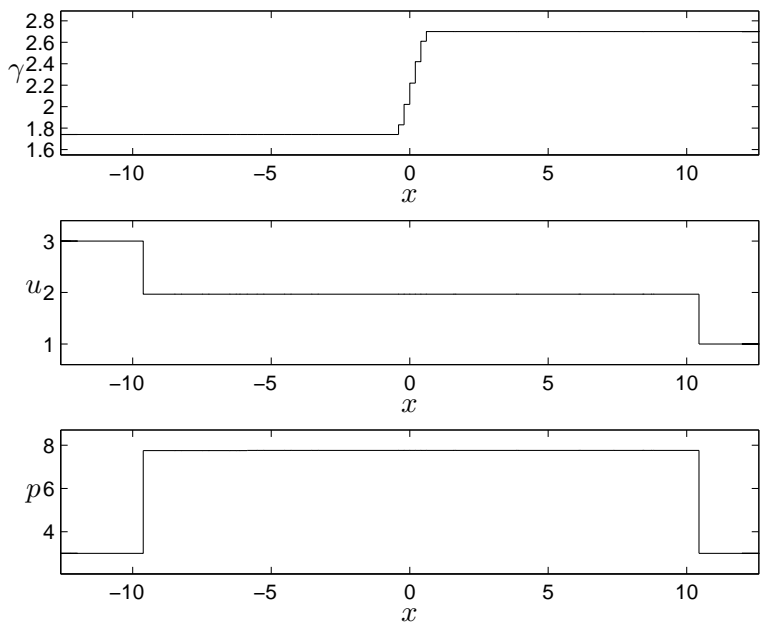

(c) $t=2$.

FiguRE 26. $U^{\delta}(\cdot, t)$ at different times $t$ for Example 4.3 . 


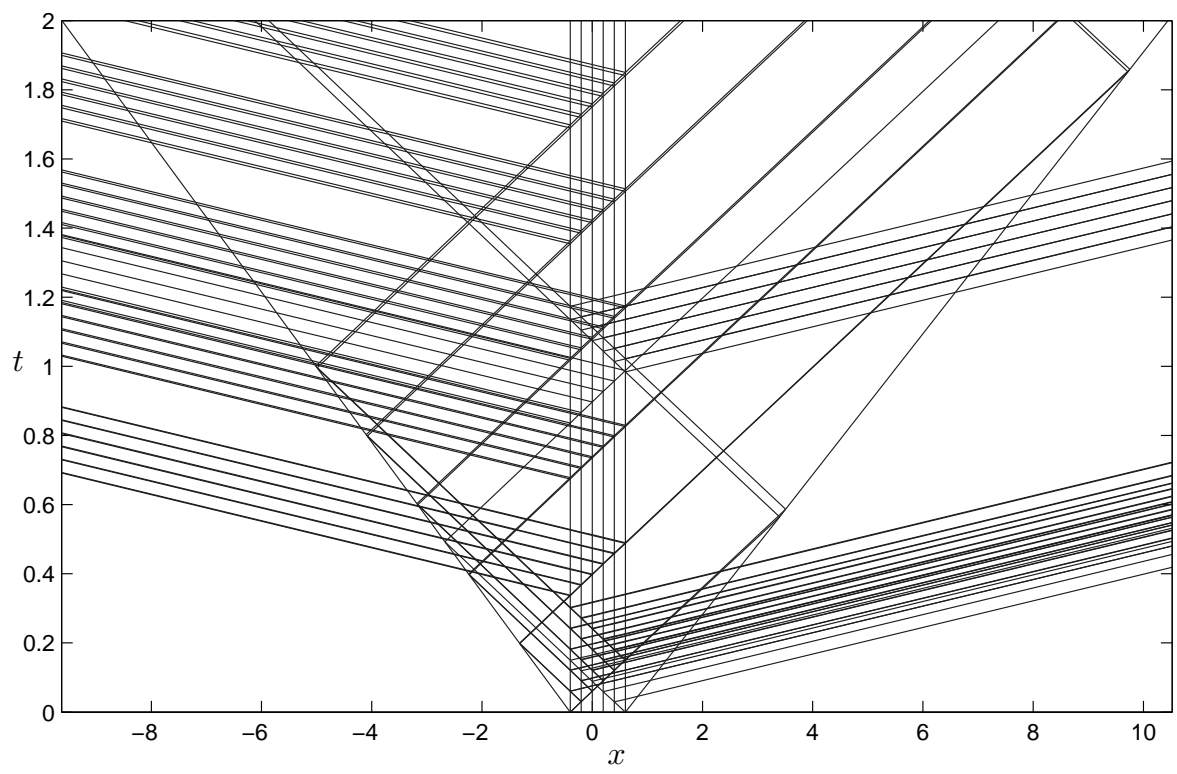

FIGURE 27. The solution $U^{\delta}(x, t)$ in the $(x, t)$-plane for Example 4.3

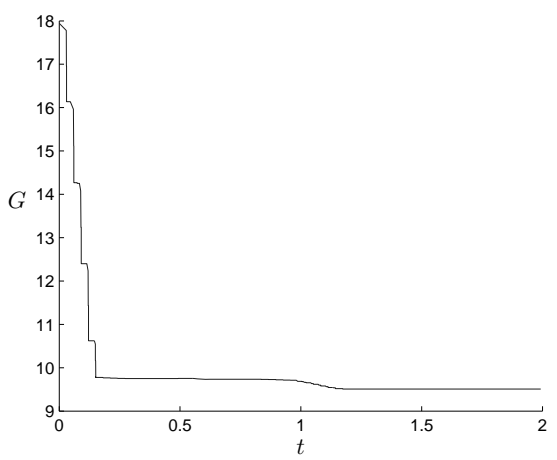

FIGURE 28. $G$ for Example 4.3 .

[17] T.-P. Liu. Solutions in the large for the equations of nonisentropic gas dynamics. Indiana Univ. Math. J-, 26(1):147-177, 1977.

[18] T. P. Liu and J. A. Smoller. On the vacuum state for the isentropic gas dynamics equations. Adv. in Appl. Math., 1(4):345-359, 1980.

[19] Y. Lu. Hyperbolic Conservation Laws and the Compensated Compactness Method. Shapman\&Hall/CRC, 2003.

[20] T. Nishida. Global solution for an initial boundary value problem for a quasilinear hyperbolic system. Proc. Japan Acad., 44:642-646, 1968.

[21] T. Nishida and J. A. Smoller. Solution in the large for some nonlinear hyperbolic conservation laws. Comm. Pure Appl. Math., 26:183-200, 1973.

[22] Y.-J. Peng. Solutions faibles globales pour l'quation d'Euler d'un fluide compressible avec de grandes donnees initales. Comm. Partial Differential Equations, 17(1-2):161-187, 1992.

[23] Y.-J. Peng. Solutions faibles globales pour un modèle d'écoulements diphasiques. Ann. Scuola Norm. Sup. Pisa Cl. Sci. (4), 21(4):523-540, 1994.

[24] J. Smoller. Shock Waves and Reaction-Diffusion Equations. Springer-Verlag, second edition, 1994. 
[25] J. B. Temple. Solution in the large for the nonlinear hyperbolic concervation laws of gas dynamics. J. Differential Equations, 41:96-161, 1981.

[26] B. Temple and R. Young. The large time stability of sound waves. Comm. Math. Phys., 179(2):417-466, 1996.

[27] D. H. Wagner. Equivalence of the Euler and Lagrangian equations of gas dynamics for weak solutions. J. Differential Equations, 68(1):118-136, 1987.

[28] W.-A. Yong. A simple approach to Glimm's interaction estimates. Appl. Math. Lett., 12(2):29-34, 1999.

[29] B. D. Wissmann. Global soutions to the ultra-relativistic Euler equations. arXiv:0705.1333v1 [math.AP] 9 May 2007.

(Holden)

Department of Mathematical Sciences, Norwegian University of Science and TechnolOGY, NO-7491 TRONDHEIM, NORWAY, AND

Centre of Mathematics for Applications, University of Oslo, P.O. Box 1053, Blindern, NO-0316 OSLO, NORWAY

E-mail address: holden@math.ntnu.no

$U R L$ : www.math.ntnu.no/ ${ }^{\text {holden/ }}$

(Risebro)

Centre of Mathematics for Applications, University of Oslo, P.O. Box 1053, Blindern, NO-0316 OsLo, NoRWAY

E-mail address: nilshr@math.uio.no

$U R L$ : folk.uio.no/nilshr/

(Sande)

Department of Mathematical Sciences, Norwegian University of Science and Technology, NO-7491 Trondheim, Norway

E-mail address: hildes@math.ntnu.no

URL: www.math.ntnu.no/ hildes/ 